\title{
Classical solvability of multidimensional two-phase Stefan problem for degenerate parabolic equations and Schauder's estimates for a degenerate parabolic problem with dynamic boundary conditions
}

\author{
To the memory of Professor B.V.Bazaliy
}

\section{S. P. Degtyarev}

\begin{abstract}
We consider multidimensional two-phase Stefan problem for degenerate parabolic equations of the porous medium type in classes of smooth functions. First we find a natural Hölder class for the Dirichlet boundary conditions in the initial boundary boundary problem for a degenerate parabolic equation of second order. This class then is used to obtain the Schauder estimates for a degenerate parabolic equation with dynamic boundary conditions. As a result we prove the existence locally in time of a smooth solution for Stefan problem for degenerate parabolic equations.
\end{abstract}

Mathematics Subject Classification (2010). Primary 35R35; Secondary 35K65, 35R37, 35K60.

Keywords. Free boundary, Stefan problem, Classical solvability, Porous medium equation, Degenerate parabolic equations, Dynamic boundary conditions, Schauder estimates.

\section{Introduction and the main result}

Classical solvability of Stefan problem for uniformly parabolic equations has been well studied - see, for example, papers [1-13] and the references therein. At the same time, as has long been known, the heat transfer model based on uniformly parabolic equations has some properties, which can not be observed in the reality. In particular, the infinite speed of propagation of disturbances takes place for uniformly parabolic equations. We also know that more accurate model of the heat transfer is the model on the base of degenerate parabolic equations such as the equations of the form 


$$
u_{t}(x, t)-\nabla\left(|u|^{m-1} \nabla u(x, t)\right)=f(x, t),
$$

where $m>1$. As it is known, a short formulation of classical Stefan problem for equation (1.1) is the equation

$$
(\beta(u))_{t}-\nabla\left(|u|^{m-1} \nabla u(x, t)\right)=0,
$$

where $\beta(u)$ is the discontinuous function of the form

$$
\beta(u)=\left\{\begin{array}{cc}
u, & u \leq 0 \\
u+k, & u>0,
\end{array}\right.
$$

where $k>0$ is the latent heat of fusion (crystallization) and Eq. (1.2) is considered in the sense of distributions. We stress that for quasilinear Eq. (1.2) the main unknown is, in fact, the interface $\{u=0\}$. Outside of this interface the solution of (1.2) is smooth in view of well-known local theory of uniformly parabolic equations.

In its generalised formulation this problem was considered in a number of papers, from which we mention, for example, [14-20]. Note that the generalised formulation gives weak solutions of the problem and omit the question about properties of the interface $\{u=0\}$.

As for the smooth solutions, in the most simple case of one spatial variable such problem for degenerate parabolic equations was considered in [21-25], where the existence of classical solutions was proved. The one-dimensional case is fundamentally different from the multidimensional setting. In the case of one spatial variable the interface $\{u=0\}$ is reduced to a point at each moment of time. Therefore, there are no questions about it's properties with respect to the spatial variables. At the same time the existence of the smooth interface for the solution of (1.2) in the multidimensional setting is still unknown.

However, this question is important, in our opinion, not only for studying of the melting-crystallisation mathematical models, where Stefan problem originates. It is well-known that Stefan problem arises as a mathematical model in very many fields of scientific investigations. We do not give broad examples of references to the relevant literature since they are very numerous. Let us mention however two areas of scientific research, where Stefan problem arises.

The first area is the mathematical biology. This includes the population theory, medicine, predator-pray models - see, for example [10,26-29]. At the same time degenerate equations are increasingly used in models of mathematical biology [30,31]. This leads to the consideration of Stefan type models for degenerate operators.

The second important area we mention is the area of mathematical finance including the mathematical models of stock markets. Here the optimal stopping problems for the corresponding stochastic processes are expressed by means of Stefan problem $[32,33]$. It is known by now that many stochastic processes from mathematical finance obey degenerate stochastic differential equations. And again, the optimal stopping problems for such processes lead to the consideration of Stefan problem for degenerate operators.

Besides, there are two more points of interest to consider Stefan problem for degenerate equations in classes of smooth functions. In this paper we 
are going to consider Stefan problem as a nonlinear equation in some Banach spaces and to apply a variant of classical inverse function theorem. So we need to consider the corresponding Frechet derivative of a nonlinear mapping, which is defined by Stefan problem. This leads to the investigation of a linear initial-boundary value problem with a dynamic boundary condition for linear degenerate parabolic equations. This linear problem is important by itself in our opinion. In recent years, the study of boundary value problems with dynamic boundary conditions is attracting increasing attention [34-41]. Note that to our knowledge there are no papers containing Schauder's estimate of solutions to such problems for degenerate equations, which is the subject of our interest in this paper-Sect. 6, Theorem 6.2, Theorem 6.4. Finally, we use in this paper another new result-Theorem 2.7. Here we prove solvability and Schauder estimates for the solution of the inhomogeneous initial-boundary Dirichlet problem for a linear degenerate parabolic equation. Such a problem with zero boundary conditions has been studied previously in [42]. We essentially use the results of this paper.

Thus the aim of this paper is a proof of classical solvability of the Stefan problem of the type (1.2) for a degenerate equation in the multidimensional setting, that is, the proof of the existence of the smooth surface $\{u=0\}$, and the proof of a smoothness of the solution up to the interface.

We now formulate the precise statement of Stefan problem in a more expanded than (1.2) form as it is custom in the theory of free boundary problems. Let $\Omega$ be a doubly connected domain in $R^{N}$, whose boundary consists of two smooth connected surfaces $\Gamma^{+}$and $\Gamma^{-}$without self-intersections, $\partial \Omega=\Gamma^{+} \cup \Gamma^{-}$. Suppose further, that $\Gamma$ is a given smooth surface without self-intersections lying strictly between $\Gamma^{+}$and $\Gamma^{-}$and separating the domain $\Omega$ into two doubly connected sub-domains $\Omega^{+}$and $\Omega^{-}$, so that $\partial \Omega^{ \pm}=\Gamma \cup \Gamma^{ \pm}$. For a fixed $T>0$ denote $\Omega_{T}=\Omega \times(0, T), \Omega_{T}^{ \pm}=\Omega^{ \pm} \times(0, T), \Gamma_{T}=\Gamma \times[0, T]$, $\Gamma_{T}^{ \pm}=\Gamma^{ \pm} \times[0, T]$.

Denote by $S_{T}$ a smooth surface in the cylindrical domain $\Omega_{T}$ in space $(y, \tau) \in R^{N} \times[0, T]$ such that at $\tau=0$ it coincides with $\Gamma, S_{T} \cap\{\tau=0\}=\Gamma$. Let also $S_{T}$ does not intersect surfaces $\Gamma_{T}^{ \pm}$and divides $\Omega_{T}$ into two sub-domains $Q_{T}^{+}$and $Q_{T}^{-}$. The lateral boundaries of these domains consist of $S_{T}$ and $\Gamma_{T}^{ \pm}$ respectively. The surface $S_{T}$ is unknown and has to be determined together with functions $u^{+}(y, \tau)$ and $u^{-}(y, \tau)$, which are defined in $Q_{T}^{ \pm}$respectively. The triple $\left(S_{T}, u^{+}, u^{-}\right)$must satisfy the following conditions (we denote the independent variables by $(y, \tau)$ in view of a subsequent change of variables):

$$
\begin{gathered}
\frac{\partial u^{ \pm}}{\partial \tau}-\nabla_{y}\left(a^{ \pm}\left|u^{ \pm}\right|^{m-1} \nabla_{y} u^{ \pm}\right)=0, \quad(y, \tau) \in Q_{T}^{ \pm}, \\
u^{+}(y, \tau)=u^{-}(y, \tau)=0, \quad(y, \tau) \in S_{T}, \\
a^{+} \sum_{i=1}^{N} \cos \left(\vec{N}, y_{i}\right)\left|u^{+}\right|^{m-1} u_{y_{i}}^{+}-a^{-} \sum_{i=1}^{N} \cos \left(\vec{N}, y_{i}\right)\left|u^{-}\right|^{m-1} u_{y_{i}}^{-} \\
=k \cos (\vec{N}, \tau), \quad(y, \tau) \in S_{T}, \\
u^{ \pm}(y, \tau)=g^{ \pm}(y, \tau),(y, \tau) \in \Gamma_{T}^{ \pm},
\end{gathered}
$$




$$
u^{ \pm}(y, 0)=u_{0}^{ \pm}(y) .
$$

Here $m>1, k>0, a^{ \pm}>0$ are given constants, $\vec{N}$ is the unite normal to $S_{T}$ with the direction into $Q_{T}^{+}, g^{+}(y, \tau), g^{-}(y, \tau), u_{0}^{+}(y), u_{0}^{-}(y)$ are given functions. We suppose that

$$
\pm g^{ \pm}(y, \tau) \geq \nu>0,(y, \tau) \in \Gamma_{T}^{ \pm} ; \quad \pm u_{0}^{ \pm}(y)>0, y \in \Omega^{ \pm}, u_{0}^{ \pm}(y)=0, y \in \Gamma,
$$

where $\nu$ is some positive constant: here and below we denoted by the same symbols $\nu, \mu, C$ all absolute constants or constants that depend only on the given data of the problem. Note that conditions (1.4), (1.5) arise from equation (1.2) and they are the three independent conditions at the unknown boundary $S_{T}$.

We introduce some weighted functional spaces to formulate the smoothness conditions on the data of the problem. First of all we use the standard Hölder spaces $H^{l+\delta}(\bar{\Omega}) \equiv C^{l+\delta}(\bar{\Omega}), \delta \in(0,1), l \in \mathbb{N} \cup\{0\}$ with the norm $|u| \frac{(l+\delta)}{\Omega}$. These spaces are introduced in [43]. We also use spaces $H^{l+\delta, \frac{l+\delta}{2}}\left(\overline{\Omega_{T}}\right) \equiv C^{l+\delta, \frac{l+\delta}{2}}\left(\overline{\Omega_{T}}\right)$ of functions of $(y, \tau)$ with the norm $|u|_{\overline{\Omega_{T}}}^{(l+\delta)}$. In [43] the surfaces of the corresponding classes are also defined. We assume that the surfaces $\Gamma, \Gamma^{ \pm}$belong to the following classes

$$
\Gamma, \Gamma^{ \pm} \in H^{4+\gamma}
$$

with some $0<\gamma<1$. At the same time we suppose that the functions $g^{ \pm}$in (1.6) are such that

$$
h^{ \pm}(y, \tau) \equiv\left|g^{ \pm}(y, \tau)\right|^{m-1} g^{ \pm}(y, \tau) \in H^{4+\gamma, \frac{4+\gamma}{2}}\left(\Gamma_{T}^{ \pm}\right) .
$$

Suppose further that $d^{+}(y)$ is a given function from $H^{2+\gamma}\left(\overline{\Omega^{+}}\right)$such that it models the distance from a point $y \in \overline{\Omega^{+}}$to the surface $\Gamma$, that is,

$$
\nu \leq d^{+}(y) / \operatorname{dist}(y, \Gamma) \leq \nu^{-1} .
$$

Note that such function can be taken, for example, as the solution of the following problem

$$
\begin{gathered}
\Delta d^{+}(y)=-1, y \in \Omega^{+}, \\
d^{+}(y)=0, y \in \Gamma, \quad d_{+}(y)=1, y \in \Gamma^{+} .
\end{gathered}
$$

Let $d^{-}(y)$ is an analogous function for the domain $\overline{\Omega^{-}}$.

Denote here and below

$$
\alpha=\frac{m-1}{m} \in(0,1),
$$

where $m>1$ is the exponent from equation (1.3).

We will use the spaces $C_{s}^{2+\gamma, \frac{2+\gamma}{2}}\left(\bar{\Omega}_{T}^{ \pm}\right)$from paper [42] (they are analogous to the corresponding spaces from [44]), where $0<\gamma<\alpha$ is some exponent. Here we require

$$
0<\gamma<\alpha .
$$

These spaces are defined in the following way. First we define the spaces $C_{s}^{2+\gamma}\left(R_{+T}^{N}\right)$ in the domain 


$$
R_{+T}^{N}=R_{+}^{N} \times[0, T], \quad R_{+}^{N}=\left\{x=\left(x^{\prime}, x_{N}\right): x_{N} \geq 0, x^{\prime} \in R^{N-1}\right\} .
$$

Define a distance between points $x, \bar{x} \in R_{+}^{N}$ according to the following formula

$$
s(x, \bar{x})=\frac{|x-\bar{x}|}{x_{N}^{\alpha / 2}+\bar{x}_{N}^{\alpha / 2}+\left|x^{\prime}-\bar{x}^{\prime}\right|^{\alpha / 2}} .
$$

Define further a Hölder constant of a function $u(x, t)$ with respect to the variable $x$ according to the distance from (1.15)

$$
H_{s, R_{+T}^{N}}^{\gamma}(u) \equiv \sup _{(x, t),(\bar{x}, t) \in R_{+T}^{N}} \frac{|u(x, t)-u(\bar{x}, t)|}{s(x, \bar{x})^{\gamma}} .
$$

Denote by $C_{s}^{\gamma, \gamma / 2}\left(R_{+T}^{N}\right)$ the space of functions $u(x, t)$ with the finite norm

$$
|u|_{C_{s}^{\gamma, \gamma / 2}\left(R_{+T}^{N}\right)} \equiv|u|_{s, R_{+T}^{N}}^{(\gamma)} \equiv|u|_{R_{+T}^{N}}^{(0)}+H_{s, R_{+T}^{N}}^{\gamma}(u)+\langle u\rangle_{t, R_{+T}^{N}}^{\left(\frac{\gamma}{2}\right)},
$$

where $\langle u\rangle_{t, R_{+T}^{N}}^{\left(\frac{\gamma}{2}\right)}$ is the Hölder constant with respect to $t$ with the exponent $\gamma / 2$ of a function $u(x, t),|u|_{R_{+T}^{N}}^{(0)}=\max _{R_{+T}^{N}}|u|$.

Define further the space $C_{s}^{2+\gamma, \frac{2+\gamma}{2}}\left(R_{+T}^{N}\right)$ as the Banach space of functions $u(x, t)$ with the finite norm

$$
\begin{gathered}
|u|_{C_{s}^{2+\gamma, \frac{2+\gamma}{2}}\left(R_{+T}^{N}\right)} \equiv|u|_{s, R_{+T}^{N}}^{(2+\gamma)} \equiv|u|_{C_{s}^{\gamma, \gamma / 2}\left(R_{+T}^{N}\right)}+\sum_{i=1}^{N}\left|u_{x_{i}}\right|_{C_{s}^{\gamma, \gamma / 2}\left(R_{+T}^{N}\right)} \\
+\left|u_{t}\right|_{C_{s}^{\gamma, \gamma / 2}\left(R_{+T}^{N}\right)}+\sum_{i, j=1}^{N}\left|x_{N}^{\alpha} u_{x_{i} x_{j}}\right|_{C_{s}^{\gamma, \gamma / 2}\left(R_{+T}^{N}\right)}
\end{gathered}
$$

Finally, the spaces $C_{s}^{\gamma, \gamma / 2}\left(\bar{\Omega}_{T}^{ \pm}\right)$and $C_{s}^{2+\gamma, \frac{2+\gamma}{2}}\left(\bar{\Omega}_{T}^{ \pm}\right)$are defined as the spaces of functions $u(x, t)$ with the property, that in some neighborhood of $\Gamma_{T}$ after the corresponding change of variables functions $u(x, t)$ belong to the space $C_{s}^{\gamma, \gamma / 2}\left(R_{+T}^{N}\right)$ or to the space $C_{s}^{2+\gamma, \frac{2+\gamma}{2}}\left(R_{+T}^{N}\right)$ correspondingly. Out of some neighborhood of $\Gamma_{T}$ such functions $u(x, t)$ belong to the standard spaces $C^{\gamma, \gamma / 2}\left(\bar{\Omega}_{T}^{ \pm}\right)$or $C^{2+\gamma, \frac{2+\gamma}{2}}\left(\bar{\Omega}_{T}^{ \pm}\right)$. In particular, for a function $u \in C_{s}^{2+\gamma, \frac{2+\gamma}{2}}\left(\bar{\Omega}_{T}^{ \pm}\right)$ the following norm is finite

$$
\left|\left(d^{ \pm}\right)^{\alpha} u_{x_{i} x_{j}}\right|_{C_{s}^{\gamma, \gamma / 2}\left(\bar{\Omega}_{T}^{ \pm}\right)}<\infty, \quad i, j=\overline{1, N} .
$$

In the case of functions $u(x)$ of the variable $x$ only, $x \in \bar{\Omega}^{ \pm}$, the spaces $C_{s}^{\gamma}\left(\bar{\Omega}^{ \pm}\right)$and $C_{s}^{2+\gamma}\left(\bar{\Omega}^{ \pm}\right)$are defined completely analogous.

We will use also some more general than the spaces $H^{l, l / 2} \equiv C^{l, l / 2}$ but standard anisotropic spaces of smooth functions. Namely, we will use the spaces

$$
C^{l_{1}, l_{2}}\left(\bar{\Omega}_{T}^{ \pm}\right), \quad C^{l_{1}, l_{2}}\left(\Gamma_{T}\right),
$$

where $l_{1}, l_{2}$ are non-integer positive numbers. Such spaces are defined in [45], for example. They consist from functions that are smooth with respect to $x$ and $t$ up to the orders $l_{1}$ and $l_{2}$ respectively. This means that the following norm is finite 


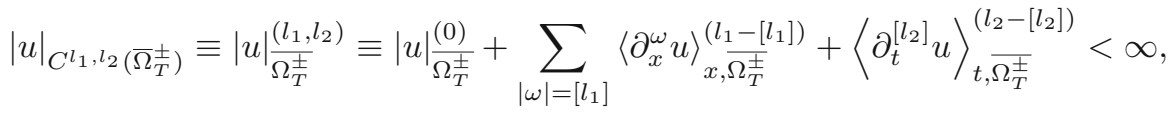

where $\omega=\left(\omega_{1}, \ldots, \omega_{N}\right)$ is a multiindex, $|\omega|=\omega_{1}+. .+\omega_{N}$,

$$
\partial_{x}^{\omega} u=\frac{\partial^{|\omega|} u}{\partial_{x_{1}}^{\omega_{1}} \ldots \partial_{x_{N}}^{\omega_{N}}} .
$$

In fact, the functions from such spaces possess the property, that their derivatives with respect to $x$ are smooth with respect to $t$ and their derivatives with respect to $t$ are smooth with respect to $x$. More precisely, if $k_{1}$ is a multiindex, that is, $k_{1}=\left(k_{1,1}, k_{1,2}, \ldots, k_{1, N}\right)$ where $k_{1, i}$ are nonnegative integers, and $k_{2}$ is a nonnegative integer, then the function $\partial_{x}^{k_{1}} \partial_{t}^{k_{2}} u(x, t)$ belongs to the space $C^{m_{1}, m_{2}}\left(\bar{\Omega}_{T}^{ \pm}\right)$with the exponents $m_{i}=\mu l_{i}$, where

$$
\mu=1-\frac{\left|k_{1}\right|}{l_{1}}-\frac{k_{2}}{l_{2}}
$$

(see [45]), and also

$$
\left|\partial_{x}^{k_{1}} \partial_{t}^{k_{2}} u\right|_{C^{m_{1}, m_{2}\left(\bar{\Omega}_{T}^{ \pm}\right)}} \leq C|u|_{C^{l_{1}, l_{2}\left(\bar{\Omega}_{T}^{ \pm}\right)}} .
$$

In addition, we will use the spaces with zero at the bottom of their notation, that is, the spaces (compare [43], Chapter IV)

$$
C_{0, s}^{2+\gamma, \frac{2+\gamma}{2}}\left(\bar{\Omega}_{T}^{ \pm}\right), C_{0}^{l_{1}, l_{2}}\left(\bar{\Omega}_{T}^{ \pm}\right), C_{0, s}^{\gamma, \frac{\gamma}{2}}\left(\bar{\Omega}_{T}^{ \pm}\right), C_{0}^{l_{1}, l_{2}}\left(\Gamma_{T}\right) .
$$

Such notation means the closed subspace of the corresponding space with the following property: all functions from this subspace vanish at $t=0$ together with all their derivatives with respect to $t$ up to the highest possible order in the corresponding space.

Let here and throughout below the exponent $\beta \in(0,1)$ is connected to the exponent $\gamma$ by the equality

$$
\beta=\gamma\left(1-\frac{\alpha}{2}\right) .
$$

In particular, we will use the spaces $C^{2+\beta-\alpha, 1+\frac{\gamma}{2}}\left(\bar{\Omega}_{T}^{ \pm}\right)$and $C^{2+\beta-\alpha, 1+\frac{\gamma}{2}}\left(\Gamma_{T}\right)$. These spaces consist of functions $u(x, t)$ with smoothness with respect to $x$ up to the order $2+\beta-\alpha$ and with smoothness with respect to $t$ up to the order $1+\gamma / 2$, that is,

$$
\sum_{i=1}^{N}\left\langle u_{x_{i}}\right\rangle_{x, \bar{\Omega}_{T}^{ \pm}}^{(1+\beta-\alpha)}+\left\langle u_{t}\right\rangle_{t, \bar{\Omega}_{T}^{ \pm}}^{\left(\frac{\gamma}{2}\right)}<\infty .
$$

In view of $(1.22)$ it is easy to calculate that

$$
1+\frac{\gamma}{2}=\frac{2+\beta-\alpha}{2-\alpha},
$$

so we also use the following notation for the mentioned above spaces

$$
\begin{aligned}
& C^{2+\beta-\alpha, \frac{2+\beta-\alpha}{2-\alpha}}\left(\bar{\Omega}_{T}^{ \pm}\right)=C^{2+\beta-\alpha, 1+\frac{\gamma}{2}}\left(\bar{\Omega}_{T}^{ \pm}\right), \\
& C^{2+\beta-\alpha, \frac{2+\beta-\alpha}{2-\alpha}}\left(\Gamma_{T}\right)=C^{2+\beta-\alpha, 1+\frac{\gamma}{2}}\left(\Gamma_{T}\right) .
\end{aligned}
$$


Further, we suppose that the initial conditions in (1.7) are such that

$$
v_{0}^{ \pm}(y) \equiv\left|u_{0}^{ \pm}(y)\right|^{m-1} u_{0}^{ \pm}(y) \in C_{s}^{2+\gamma^{\prime}}\left(\overline{\Omega^{ \pm}}\right),
$$

where $\gamma^{\prime}>\gamma$. Besides, we suppose that

$$
\frac{\partial v_{0}^{ \pm}(y)}{\partial \vec{n}} \geq \nu>0, \quad y \in \Gamma
$$

where $\vec{n}$ is the normal vector to the surface $\Gamma$, which is directed into $\Omega^{+}$.

We will show below, that the free (unknown) boundary $S_{T}$ in (1.4), (1.5) can be parameterized in terms of its deviation from the given surface $\Gamma_{T}=\Gamma \times[0, T]$. We follow to [2] to give the exact formulation. Let $\omega=\left(\omega_{1}, \ldots, \omega_{N-1}\right)$ is some local curvilinear coordinates in a domain $\Theta$ on $\Gamma$. In some small neighbourhood $\mathcal{N}$ in $R^{N}$ of the surface $\Gamma$ we introduce the coordinates $(\omega, \lambda)$ in the way that for any $x \in \mathcal{N}$ we have the following unique representation

$$
x=x^{\prime}(x)+\vec{n}\left(x^{\prime}(x)\right) \lambda \equiv x(\omega)+\vec{n}(\omega) \lambda,
$$

where $x^{\prime}(x)=x(\omega)$ is the point in the domain $\Theta$ on the surface $\Gamma$ with the coordinates $\omega, \vec{n}(\omega)$ - the normal vector to $\Gamma$ at the point $x(\omega)$ with the direction into $\Omega^{+}$. The coordinate $\lambda \in R$ means, in fact, the deviation of a point $x$ from $\Gamma, \pm \lambda>0$ for $x \in \Omega^{ \pm}$. We assume that the neighbourhood $\mathcal{N}$ of the surface $\Gamma$ is the set

$$
\mathcal{N}=\left\{x \in \Omega:|\lambda(x)|<\gamma_{0}\right\},
$$

where $\gamma_{0}$ is sufficiently small and will be chosen below.

Let $\rho\left(x^{\prime}, t\right) \equiv \rho(\omega, t)$ is a sufficiently small and regular function and $\rho\left(x^{\prime}, t\right) \equiv \rho(\omega, t)$ is defined on the surface $\Gamma_{T}$. Let us note that here and in what follows we use the notation $\rho(\omega, t)$ with the argument $\omega$ instead of $\rho\left(x^{\prime}, t\right)$ for all functions on the surface $\Gamma$ if it does not cause ambiguity. We do that just for simplification of the notation, bearing in mind that in each local domain $\Theta$ on $\Gamma$ we can introduce local coordinates $\omega$. At the same time the coordinate $\lambda$ in (1.28) does not depend on a choice of local coordinates $\omega$.

We parameterize the unknown surface $S_{T}$ with the help of the unknown function $\rho(\omega, t)$ as follows

$$
S_{T} \equiv \Gamma_{\rho, T}=\left\{(x, t) \in \Omega_{T}: x=x^{\prime}+\rho\left(x^{\prime}, t\right) \vec{n}\left(x^{\prime}\right)=x(\omega)+\rho(\omega, t) \vec{n}(\omega)\right\},
$$

where $x^{\prime} \equiv x(\omega) \in \Gamma$. Note that this definition of the surface $S_{T} \equiv \Gamma_{\rho, T}$ does not depend on a choice of local coordinates $\omega$ in a particular local domain on $\Gamma$. Thus, the unknown function $\rho(\omega, t)$ means, in fact, deviation of the surface $\Gamma_{\rho, T}=S_{T}$ from the given surface $\Gamma_{T}$.

Along with $Q_{T}^{+}, Q_{T}^{-}$in (1.3) we use the notation $\Omega_{\rho, T}^{+}=Q_{T}^{+}$and $\Omega_{\rho, T}^{-}=$ $Q_{T}^{-}$for the sub-domains of the domain $\Omega_{T}$ under consideration. Let further $\rho(x, t)$ is an extension of the function $\rho(\omega, t)$ from the surface $\Gamma_{T}$ to the whole domain $\Omega_{T}$ to a function with support in the neighborhood $\mathcal{N}_{T}=\mathcal{N} \times[0, T]$ of the surface $\Gamma_{T}, \rho(x, t)=E \rho(\omega, t)$, where $E$ is some fixed extension operator. 
The way of such an extension will be described below and we will denote $\left.\rho^{ \pm} \equiv E \rho\right|_{\bar{\Omega}_{T}^{ \pm}} \equiv E^{ \pm} \rho$.

Define a mapping $e_{\rho}(x, t)$ from the domain $\overline{\Omega_{T}}$ on itself with the help of the formula $e_{\rho}:(x, t) \rightarrow(y, \tau)$, where, according to the notations of (1.28),

$$
\begin{gathered}
y=\left\{\begin{array}{ll}
x^{\prime}(x)+\vec{n}\left(x^{\prime}(x)\right)(\lambda(x)+\rho(x, t)), & x \in \mathcal{N}, \\
x, & x \in \bar{\Omega} \backslash \mathcal{N},
\end{array}, \tau=t,\right.
\end{gathered}
$$

or, with the help of the local coordinates $\omega$,

$$
\begin{gathered}
y= \begin{cases}x^{\prime}(\omega(x))+\vec{n}(\omega(x))(\lambda(x)+\rho(x, t)), & x \in \mathcal{N}, \\
x, & x \in \bar{\Omega} \backslash \mathcal{N},\end{cases} \\
\tau=t .
\end{gathered}
$$

Here $x^{\prime}(x) \in \Gamma, \omega(x), \lambda(x)$ are $(\omega, \lambda)$ - coordinates of a point $x$ in the neighbourhood $\mathcal{N}$. Note that the definition of the mapping $e_{\rho}$ does not depend on a choice of local coordinates $\omega$ on the surface $\Gamma$.

We choose $\gamma_{0}$ sufficiently small so that under the condition

$$
|\rho|_{\Gamma}^{1+\beta} \leq 2 \gamma_{0}
$$

the mapping $e_{\rho}$ is a diffeomorphism of $\overline{\Omega_{T}}$ on itself and also the mapping $e_{\rho}$ is a diffeomorphism of the domains $\overline{\Omega_{T}^{ \pm}}$on the domains $\overline{\Omega_{\rho, T}^{ \pm}}$. Let us remark that the surface $\Gamma_{\rho, T}$ is exactly the image of the surface $\Gamma_{T}$ under this mapping and the mapping $e_{\rho}(x, t)$ is the identical mapping out of the neighbourhood $\mathcal{N}_{T}$ of $\Gamma_{T}$.

About the exponents of the Hölder spaces under consideration we suppose that

$$
0<\gamma<\gamma^{\prime}<1, \quad \gamma<\min \{\alpha, 1-\alpha\}
$$

Note that under our choice of $\gamma$ the restriction $\gamma<\frac{\alpha}{1-\alpha / 2}$ is also fulfilled. The last restriction was introduced in [42] at the studying of the homogeneous initial-boundary problem for degenerate equations. We need the restriction $\gamma<1-\alpha$ to have the inequality $1+\beta-\alpha>1-\alpha>\gamma$ because this inequality implies that the first derivatives with respect to $x$ of functions from the classes $C^{2+\beta-\alpha, \frac{2+\beta-\alpha}{2-\alpha}}, C_{s}^{2+\gamma, \frac{2+\gamma}{2}}\left(\bar{\Omega}_{T}^{ \pm}\right)$are more smooth than $C^{\gamma, \gamma / 2}, C_{s}^{\gamma, \gamma / 2}$. At last, we need the restriction $\gamma<\alpha$ because under this restriction the expressions of the form $\left(d^{ \pm}(x)\right)^{\alpha} \eta(x, t)$ with a smooth function $\eta(x, t)$ belong to the corresponding space $C_{s}^{\gamma, \gamma / 2}$.

We impose the following natural agreement conditions up to the first order at $\tau=0, y \in \Gamma, \Gamma^{ \pm}$for problem (1.3)-(1.7). We suppose that the given functions $g^{+}(y, \tau), g^{-}(y, \tau), u_{0}^{+}(y), u_{0}^{-}(y)$ satisfy

$$
\begin{gathered}
\left.u_{0}^{+}(y)\right|_{\Gamma^{+}}=g^{+}(y, 0),\left.\quad u_{0}^{-}(y)\right|_{\Gamma^{-}}=g^{-}(y, 0), \\
\left.\nabla_{y}\left(a^{ \pm}\left|u_{0}^{ \pm}\right|^{m-1} \nabla_{y} u_{0}^{ \pm}\right)\right|_{\Gamma^{ \pm}}=\frac{\partial g^{ \pm}(y, 0)}{\partial t},
\end{gathered}
$$




$$
\left.\frac{1}{k}\left(a^{+} \frac{\partial v_{0}^{+}}{\partial \vec{n}}-a^{-} \frac{\partial v_{0}^{-}}{\partial \vec{n}}\right)\right|_{\Gamma}=-\left(a^{ \pm}\left|v_{0}^{ \pm}\right|^{\alpha} \nabla^{2} v_{0}^{ \pm}\right) /\left.\left(\frac{\partial v_{0}^{ \pm}}{\partial \vec{n}}\right)\right|_{\Gamma},
$$

where the functions $v_{0}^{ \pm}$are defined in (1.26) and satisfy (1.27). Note that condition (1.36) is necessary to agree conditions (1.3), (1.4), and (1.5). It is explained below in (5.1), (5.2).

Let us formulate now the main result.

Theorem 1.1. Let conditions (1.8)-(1.10), (1.33) on the data of problem (1.3)(1.7) and conditions (1.26), (1.27) are satisfied. Let also agreement conditions (1.34)-(1.36) at $\tau=0, y \in \Gamma, \Gamma^{ \pm}$are satisfied. Then there exists $T>0$ such that on the time interval $[0, T]$ problem (1.3)-(1.7) has the unique smooth solution. The unknown boundary can be represented as in (1.29) with a function $\rho(\omega, t)$ with the properties

$$
\begin{array}{r}
\rho(\omega, t) \in C^{2+\beta-\alpha, \frac{2+\beta-\alpha}{2-\alpha}}\left(\Gamma_{T}\right), \rho_{t}(\omega, t) \in C^{1+\beta-\alpha, \frac{1+\beta-\alpha}{2-\alpha}}\left(\Gamma_{T}\right), \\
\rho^{ \pm}(x, t)=E^{ \pm} \rho(\omega, t) \in C_{s}^{2+\gamma}\left(\bar{\Omega}_{T}^{ \pm}\right),
\end{array}
$$

where $\rho(x, t)=E \rho(\omega, t)$ is an extension of the function $\rho(\omega, t)$ to the domain $\overline{\Omega_{T}}$. The functions $u^{ \pm}(y, \tau)$ in $(1.3)$ are such that

$$
v^{ \pm}(x, t) \equiv\left(\left|u^{ \pm}(y, \tau)\right|^{m-1} u^{ \pm}(y, \tau)\right) \circ e_{\rho}(x, t) \in C_{s}^{2+\gamma}\left(\bar{\Omega}_{T}^{ \pm}\right) .
$$

Thus, all of the relations of problem (1.3)-(1.7) are satisfied in the classical sense.

For the proof of this theorem we use some simple assertion. This assertion is, in fact, a consequence of classical Newton's method of solving nonlinear equations in Banach spaces. Currently Newton's method itself is sufficiently custom in the free boundary problems theory. Previously this method was used in free boundary problems in a number of papers - see, for example $[2-4,6,7$, 46-49]. It seems paper [46] is the first application of Newton type iterations to a multidimensional free boundary problem. Note that the more contemporary methods of the investigation of such problems are associated with the use of semigroups theory for quasi-linear operators [50]. The semigroups method allows, in particular, to consider not only local but also global solvability of free boundary problems. However, in this paper we restrict ourselves to the local solvability of the problem and therefore we use more classical arguments. More precisely, in the contrast to $[2-4,6,7,46-49]$ we do not reproduce the whole scheme of the Newton's iterations explicitly with the corresponding estimates. Instead, we just use a variant of the classical inverse function theorem in Banach spaces (as a special case of a more general classical implicit function theorem). We formulate here these theorems. First we formulate some extended version of Theorem 1.2 from [51].

Theorem 1.2. ([51], Theorem 1.2 and it's proof). Let $U$ be open in a Banach space $H$, and let $F: U \rightarrow Y$ be continuously differentiable on $U$, where $Y$ is a Banach space. Let $x_{0} \in U$ and assume that $F^{\prime}\left(x_{0}\right): H \rightarrow Y$ is a toplinear isomorphism (i.e. invertible as a continuous linear map). Then $F$ is a local 
diffeomorphism at $x_{0}$ and there exists $d>0$ such that the inverse mapping $F^{-1}$ is defined on the ball $K_{d}=\left\{y \in Y:\left\|y-y_{0}\right\| \leq d\right\}, y_{0}=F\left(x_{0}\right)$.

Here $d=d\left(M_{1}, M_{2}, \omega_{0}\right)$ depends only on $M_{1}, M_{2}, \omega_{0}$, where

$$
\begin{gathered}
M_{1}=\left\|F^{\prime}\left(x_{0}\right)\right\|_{H \rightarrow Y}, \quad M_{2}=\left\|\left(F^{\prime}\left(x_{0}\right)\right)^{-1}\right\|_{Y \rightarrow H}, \\
\omega_{0}=\sup _{x_{1}, x_{2} \in U}\left\|F^{\prime}\left(x_{1}\right)-F^{\prime}\left(x_{2}\right)\right\|_{H \rightarrow Y} .
\end{gathered}
$$

Due to this classical theorem we have the following very simple but fundamentally important for us assertion, where $x_{0}$ serves as an approximate solution to the equation $F(x)=0$.

Corollary 1.3. Let the conditions of Theorem 1.2 are satisfied. Then there exists $\varepsilon_{0}=\varepsilon_{0}\left(M_{1}, M_{2}, \omega_{0}\right)>0$ such that if $\left\|F\left(x_{0}\right)\right\|_{Y}=\left\|y_{0}\right\|_{Y} \leq \varepsilon_{0}$, then for some $x^{*} \in U$ we have $F\left(x^{*}\right)=0$.

Proof. This corollary immediately follows from Theorem 1.2 if we choose $\varepsilon_{0}=$ $d / 2$ so that $0 \in K_{d}$.

Note that since we are going to use this corollary, the key ingredient of our proof of Theorem 1.1 is the proof of the fact that $f^{\prime}\left(x_{0}\right)$ is invertible. In our case the nonlinear mapping $f(x)$ is defined by the left-hand-sides of relations (1.3)-(1.7), so we need to consider the Frechet derivative of such mapping. This is done in Sect. 6, Theorem 6.2.

The subsequent sections of the paper are devoted to the proof of Theorem 1.1 on the base of Corollary 1.3 according to the following plan.

In Sect. 2, we first introduce an equivalent norms in the spaces $C_{s}^{\gamma, \gamma / 2}$ $\left(\bar{\Omega}_{T}^{ \pm}\right)$. This permits us to study the exact class of the traces on $\Gamma_{T}$ of functions from the class $C_{s}^{2+\gamma, \frac{2+\gamma}{2}}\left(\bar{\Omega}_{T}^{ \pm}\right)$. This allows us to extend the results of [42] about the solvability of the homogeneous initial boundary value problem for a degenerate equation to the case of the inhomogeneous problem Theorem 2.7.

These results are then used in Sect. 3 to study a model Stefan problem for degenerate equations. This model problem is one of the central points of this paper. This model problem is an initial-boundary problem with dynamic boundary conditions for degenerate equations in half-spaces. To obtain Schauder's estimates for this problem the idea of paper [53] on the application of the classical maximum principle is used. In Sects. 4 and 5 the initial problem with the unknown boundary is reduced to nonlinear problem in a fixed domain. Then the corresponding operators of the problem are linearized (to find the Frechet derivative) on some functions that extend the initial data to the domain $t>0$. These functions serve as an approximate solution $x_{0}$ in Corollary 1.3. Section 6 is devoted to the study of the linear problem with dynamic boundary conditions for degenerate equations in classes of smooth functions in arbitrary smooth domains. This gives the invertibility of the Frechet derivative of the nonlinear operator of our initial problem. The obtaining of Schauder's estimates is based on the results of Sect. 3. To prove the solvability of the linear problem, we use the parabolic regularization of the Stefan boundary condition. Note that the corresponding model problem in Sect. 3 is considered in the presence of the regularization. Finally, Sect. 7 completes the proof of Theorem 1.1 by Corollary 1.3. 
2. Auxiliary results on the spaces $C_{s}^{\gamma, \gamma / 2}\left(\bar{\Omega}_{T}^{ \pm}\right), C_{s}^{2+\gamma, \frac{2+\gamma}{2}}\left(\bar{\Omega}_{T}^{ \pm}\right)$, $C^{2+\beta-\alpha, \frac{2+\beta-\alpha}{2-\alpha}}\left(\Gamma_{T}\right)$

First we give some important inequalities for the spaces with zero in (1.21). Let $\gamma^{\prime}>\gamma, l_{1}^{\prime}>l_{1}, l_{2}^{\prime}>l_{2}$. Let also

$$
\begin{aligned}
& u_{1} \in C_{0, s}^{\gamma^{\prime}, \gamma^{\prime} / 2}\left(\bar{\Omega}_{T}^{ \pm}\right), u_{2} \in C_{0, s}^{2+\gamma^{\prime}, \frac{2+\gamma^{\prime}}{2}}\left(\bar{\Omega}_{T}^{ \pm}\right), \\
& u_{3}, v_{3} \in C_{0}^{2+\gamma, \frac{2+\gamma}{2}}\left(\bar{\Omega}_{T}^{ \pm}\right), u_{4} \in C_{0}^{l_{1}^{\prime}, l_{2}^{\prime}}\left(\bar{\Omega}_{T}^{ \pm}\right) .
\end{aligned}
$$

Then

$$
\begin{gathered}
\left|u_{1}\right|_{s, \bar{\Omega}_{T}^{ \pm}}^{(\gamma)} \leq C T^{\mu}\left|u_{1}\right|_{s, \bar{\Omega}_{T}^{ \pm}}^{\left(\gamma^{\prime}\right)}, \\
\left|u_{2}\right|_{s, \bar{\Omega}_{T}^{ \pm}}^{(2+\gamma)} \leq C T^{\mu}\left|u_{2}\right|_{\left.s, \bar{\Omega}_{T}^{ \pm}\right)}^{\left(2+\gamma^{\prime}\right)}, \\
\left|u_{3} v_{3}\right|_{s, \bar{\Omega}_{T}^{ \pm}}^{(\gamma)} \leq C T^{\mu}\left|u_{3}\right|_{s, \bar{\Omega}_{T}^{ \pm}}^{(\gamma)}\left|v_{3}\right|_{s, \overline{\Omega_{T}}}^{(\gamma)}, \\
\left|u_{4}\right|_{\bar{\Omega}_{T}^{ \pm}}^{\left(l_{1}, l_{2}\right)} \leq C T^{\mu}\left|u_{4}\right|_{\bar{\Omega}_{T}^{ \pm}}^{\left(l_{1}^{\prime}, l_{2}^{\prime}\right)},
\end{gathered}
$$

where $C, \mu$ denote some positive constants. These constants depend on $\gamma, \gamma^{\prime}$, $l_{i}, l_{i}^{\prime}$.

Inequalities (2.1)-(2.4) are well known for the spaces $C_{0}^{l_{1}, l_{2}}$ (see [43,52]), and for the spaces $C_{0, s}^{\gamma, \gamma / 2}, C_{0, s}^{2+\gamma, \frac{2+\gamma}{2}}$ they are completely analogous.

Before we proceed we need one more assertion about Hölder spaces. Let $l>0$ be non-integer and let $h>0$. Denote by $\Delta_{h} u(x)=u(x+h)-u(x)$ the operator of difference with the step $h>0$. Let further $k>l$ be an integer and let $\Delta_{h}^{k}=\left(\Delta_{h}\right)^{k}$ be the $k$-th power of the operator $\Delta_{h}$. For a function $u(x) \in C^{l}\left(R^{1}\right)$ define the following seminorms

$$
\begin{gathered}
\langle u\rangle_{R^{1}}^{(l)}=\left\langle\frac{\partial^{[l]} u}{\partial x^{[l]}}\right\rangle_{x, R^{1}}^{(l-[l])}, \\
\langle\langle u\rangle\rangle_{R^{1}}^{(l)}=\sup _{x \in R^{1}, h>0} \frac{\left|\Delta_{h}^{k} u(x)\right|}{h^{l}} .
\end{gathered}
$$

Then it follows from [54] (see also [45]) that for any $k>l$ these two seminorms are equivalent

$$
\nu_{k}\langle\langle u\rangle\rangle_{R^{1}}^{(l)} \leq\langle u\rangle_{R^{1}}^{(l)} \leq \nu_{k}^{-1}\langle\langle u\rangle\rangle_{R^{1}}^{(l)} .
$$

\subsection{An equivalent norm for the spaces $C_{s}^{\gamma}\left(\bar{\Omega}_{T}^{ \pm}\right), C_{s}^{2+\gamma}\left(\bar{\Omega}_{T}^{ \pm}\right)$}

Along with the seminorm $H_{s, R_{+T}^{N}}^{\gamma}$ from (1.16) we consider in $R_{+T}^{N}$ the following weighted seminorm

$$
H_{\alpha, R_{+T}^{N}}^{\gamma}(f)=\sup _{x, \bar{x} \in R_{+}^{N}} \frac{|f(x, t)-f(\bar{x}, t)|}{|x-\bar{x}|^{\beta}}+\sup _{x, \bar{x} \in R_{+}^{N}} \widetilde{x}_{N}^{\gamma \frac{\alpha}{2}} \frac{|f(x, t)-f(\bar{x}, t)|}{|x-\bar{x}|^{\gamma}},
$$

where $\widetilde{x}_{N}=\max \left\{x_{N}, \bar{x}_{N}\right\}$, and here and throughout without loss of generality we assume that $\bar{x}_{N} \leq x_{N}$, so $\widetilde{x}_{N}=x_{N}$. 
Lemma 2.1. The seminorms $H_{\alpha, R_{+T}^{N}}^{\gamma}(f)$ and $H_{s, R_{+T}^{N}}^{\gamma}(f)$ are equivalent.

Proof. Let the seminorm $H_{s, R_{+T}^{N}}^{\gamma}(f)$ is finite. We show that

$$
H_{\alpha, R_{+T}^{N}}^{\gamma}(f) \leq C H_{s, R_{+T}^{N}}^{\gamma}(f) .
$$

Let $\varepsilon_{0} \in(0,1)$ is small and fixed. Let $x=\left(x^{\prime}, x_{N}\right)$ and let first

$$
\left|x^{\prime}-\bar{x}^{\prime}\right| \geq \varepsilon_{0} x_{N} .
$$

Then the more

$$
|x-\bar{x}| \geq\left|x^{\prime}-\bar{x}^{\prime}\right| \geq \varepsilon_{0} x_{N}
$$

Under this condition

$$
\begin{aligned}
s(x, \bar{x}) & =\frac{|x-\bar{x}|}{x_{N}^{\alpha / 2}+\bar{x}_{N}^{\alpha / 2}+\left|x^{\prime}-\bar{x}^{\prime}\right|^{\alpha / 2}} \\
& \leq C \frac{|x-\bar{x}|}{|x-\bar{x}|^{\alpha / 2}+\bar{x}_{N}^{\alpha / 2}+\left|x^{\prime}-\bar{x}^{\prime}\right|^{\alpha / 2}} \leq C|x-\bar{x}|^{1-\frac{\alpha}{2}} .
\end{aligned}
$$

Therefore, as $\beta=\gamma\left(1-\frac{\alpha}{2}\right)$,

$$
\frac{|f(x, t)-f(\bar{x}, t)|}{|x-\bar{x}|^{\beta}} \leq C \frac{|f(x, t)-f(\bar{x}, t)|}{s(x, \bar{x})^{\gamma}} \leq C H_{s, R_{+T}^{N}}^{\gamma}(f) .
$$

Besides, from (2.9) and then from (2.10),

$$
x_{N}^{\gamma \frac{\alpha}{2}} \frac{|f(x, t)-f(\bar{x}, t)|}{|x-\bar{x}|^{\gamma}} \leq \frac{x_{N}^{\gamma \frac{\alpha}{2}}}{\left(\varepsilon_{0} x_{N}\right)^{\gamma \frac{\alpha}{2}}} \frac{|f(x, t)-f(\bar{x}, t)|}{|x-\bar{x}|^{\gamma(1-\alpha / 2)}} \leq C H_{s, R_{+T}^{N}}^{\gamma}(f) .
$$

Let now

$$
\left|x^{\prime}-\bar{x}^{\prime}\right| \leq \varepsilon_{0} x_{N}
$$

Under this condition, as it easy to see,

$$
s(x, \bar{x}) \sim C x_{N}^{-\frac{\alpha}{2}}|x-\bar{x}| .
$$

Consequently

$$
x_{N}^{\gamma \frac{\alpha}{2}} \frac{|f(x, t)-f(\bar{x}, t)|}{|x-\bar{x}|^{\gamma}} \leq C \frac{|f(x, t)-f(\bar{x}, t)|}{s(x, \bar{x})^{\gamma}} \leq C H_{s, R_{+T}^{N}}^{\gamma}(f) .
$$

To estimate, further, the unweighted Hölder constant in the definition of $H_{\alpha, R_{+T}^{N}}^{\gamma}(f)$ we consider the following two cases.

$$
\left|x_{N}-\bar{x}_{N}\right| \geq \varepsilon_{0} x_{N}
$$

then

$$
|x-\bar{x}| \geq\left|x_{N}-\bar{x}_{N}\right| \geq \varepsilon_{0} x_{N}
$$

and therefore, as it was above,

$$
s(x, \bar{x}) \leq \frac{|x-\bar{x}|}{\left(|x-\bar{x}| / \varepsilon_{0}\right)^{\alpha / 2}} \leq C|x-\bar{x}|^{1-\frac{\alpha}{2}},
$$


so that, as above,

$$
\frac{|f(x, t)-f(\bar{x}, t)|}{|x-\bar{x}|^{\beta}} \leq C \frac{|f(x, t)-f(\bar{x}, t)|}{s(x, \bar{x})^{\gamma}} \leq C H_{s, R_{+T}^{N}}^{\gamma}(f) .
$$

If now under the condition (2.12) we have

$$
\left|x_{N}-\bar{x}_{N}\right| \leq \varepsilon_{0} x_{N}
$$

then in this case

$$
|x-\bar{x}| \leq\left|x^{\prime}-\bar{x}^{\prime}\right|+\left|x_{N}-\bar{x}_{N}\right| \leq 2 \varepsilon_{0} x_{N} .
$$

Therefore, in force of (2.13),

$$
\begin{aligned}
s(x, \bar{x}) & \leq C x_{N}^{-\alpha / 2}|x-\bar{x}| \\
& \leq C x_{N}^{-\alpha / 2}\left(2 \varepsilon_{0} x_{N}\right)^{\alpha / 2}|x-\bar{x}|^{1-\alpha / 2}=C|x-\bar{x}|^{1-\alpha / 2} .
\end{aligned}
$$

Consequently, in this case

$$
\frac{|f(x, t)-f(\bar{x}, t)|}{|x-\bar{x}|^{\beta}} \leq C \frac{|f(x, t)-f(\bar{x}, t)|}{s(x, \bar{x})^{\gamma}} \leq C H_{s, R_{+T}^{N}}^{\gamma}(f) .
$$

Estimate (2.7) follows now from (2.10), (2.11), (2.14), (2.15), and (2.19).

Further, let now the seminorm $H_{\alpha, R_{+T}^{N}}^{\gamma}(f)$ is finite. Let us prove the following estimate

$$
H_{s, R_{+T}^{N}}^{\gamma}(f) \leq C H_{\alpha, R_{+T}^{N}}^{\gamma}(f)
$$

Let first

$$
\left|x^{\prime}-\bar{x}^{\prime}\right| \leq \varepsilon_{0} x_{N}, \quad x_{N}>0 .
$$

Then

$$
s(x, \bar{x}) \geq \nu \frac{|x-\bar{x}|}{x_{N}^{\alpha / 2}}
$$

and consequently

$$
\frac{|f(x, t)-f(\bar{x}, t)|}{s(x, \bar{x})^{\gamma}} \leq C x_{N}^{\gamma \alpha / 2} \frac{|f(x, t)-f(\bar{x}, t)|}{|x-\bar{x}|^{\gamma}} \leq C H_{\alpha, R_{+T}^{N}}^{\gamma}(f) .
$$

In the particular case $x_{N}=0$ we have $\bar{x}_{N}=0$ and therefore

$$
s(x, \bar{x})=\left|x^{\prime}-\bar{x}^{\prime}\right|^{1-\alpha / 2}=|x-\bar{x}|^{1-\alpha / 2},
$$

and so again

$$
\frac{|f(x, t)-f(\bar{x}, t)|}{s(x, \bar{x})^{\gamma}}=\frac{|f(x, t)-f(\bar{x}, t)|}{|x-\bar{x}|^{\beta}} \leq C H_{\alpha, R_{+T}^{N}}^{\gamma}(f) .
$$

Let now we have

$$
\left|x^{\prime}-\bar{x}^{\prime}\right| \geq \varepsilon_{0} x_{N}
$$

Then

$$
s(x, \bar{x}) \geq \nu \frac{|x-\bar{x}|}{\left|x^{\prime}-\bar{x}^{\prime}\right|^{\alpha / 2}} \geq \nu|x-\bar{x}|^{1-\alpha / 2},
$$


and consequently

$$
\frac{|f(x, t)-f(\bar{x}, t)|}{s(x, \bar{x})^{\gamma}} \leq C \frac{|f(x, t)-f(\bar{x}, t)|}{|x-\bar{x}|^{\beta}} \leq C H_{\alpha, R_{+T}^{N}}^{\gamma}(f) .
$$

Thus, (2.20) follows from (2.22), (2.23), (2.25). And so the equivalence of the seminorms $H_{s, R_{+T}^{N}}^{\gamma}(f)$ and $H_{\alpha, R_{+T}^{N}}^{\gamma}(f)$ is proved.

From this lemma it follows that the norm in the space $C_{s}^{\gamma}\left(R_{+T}^{N}\right)$ may be given in the form

$$
|u|_{C_{s}^{\gamma, \gamma / 2}\left(R_{+T}^{N}\right)}=|u|_{R_{+T}^{N}}^{(0)}+H_{\alpha, R_{+T}^{N}}^{\gamma}(u)+\langle u\rangle_{t, R_{+T}^{N}}^{\gamma / 2} .
$$

Bearing in mind the local straightening of the boundary $\Gamma$, for the case of arbitrary domains $\bar{\Omega}_{T}^{ \pm}$, the norm in the space $C_{s}^{\gamma, \gamma / 2}\left(\bar{\Omega}_{T}^{ \pm}\right)$may be explicitly written as

$$
|u|_{C_{s}^{\gamma}\left(\bar{\Omega}_{T}^{ \pm}\right)}=|u|_{\bar{\Omega}_{T}^{ \pm}}^{(0)}+\langle u\rangle_{x, \bar{\Omega}_{T}^{ \pm}}^{(\beta)}+\sup _{x, \bar{x} \in \bar{\Omega}^{ \pm}}\left(\widetilde{d^{ \pm}(x, \bar{x})}\right)^{\gamma \alpha / 2} \frac{|u(x, t)-u(\bar{x}, t)|}{|x-\bar{x}|^{\gamma}}+\langle u\rangle_{t, \bar{\Omega}_{T}^{ \pm}}^{\gamma / 2},
$$

where the functions $d^{ \pm}(x)$ were introduced in the previous section in (1.11) and they model the distance to the boundary $\Gamma, \widehat{d^{ \pm}(x, \bar{x})}=\max \left\{d^{ \pm}(x), d^{ \pm}(\bar{x})\right\}$.

Quite similar, in terms of (2.28) and (1.19) we may explicitly define the norm (1.18) in the space $C_{s}^{2+\gamma}\left(\bar{\Omega}_{T}^{ \pm}\right)$.

\subsection{The traces of functions from $C_{s}^{2+\gamma, \frac{2+\gamma}{2}}\left(\bar{\Omega}_{T}^{ \pm}\right)$on $\Gamma_{T}$}

Note first that the results of this and the next subsections are generalisations of those in [55] for elliptic case and for some another weighted spaces.

Note further that according to our assumptions the surface $\Gamma$ is smooth, so we can use the local straightening of this surface to study the locally defined classes $C_{s}^{2+\gamma, \frac{2+\gamma}{2}}\left(\bar{\Omega}_{T}^{ \pm}\right)$. Therefore, it is sufficient to consider the case of the halfspace. So we consider a function $u(x, t)$ from the space $C_{s}^{2+\gamma, \frac{2+\gamma}{2}}\left(R_{+T}^{N}\right)$ with finite support in $R_{+T}^{N}=R_{+}^{N} \times[0, T]$ and study its trace at $x_{N}=0$.

Lemma 2.2. Let a function $u(x, t)$ has finite support and $u(x, t) \in C^{2+\gamma, \frac{2+\gamma}{2}}$ $\left(R_{+T}^{N}\right), 0<\gamma<\alpha, \beta=\gamma(1-\alpha / 2)$. Then the function $v\left(x^{\prime}, t\right)=u\left(x^{\prime}, 0, t\right) \in$ $C^{2+\beta-\alpha, \frac{2+\beta-\alpha}{2-\alpha}}\left(R_{T}^{N-1}\right)=C^{2+\beta-\alpha, \frac{2+\gamma}{2}}\left(R_{T}^{N-1}\right)$ and

$$
\left|v\left(x^{\prime}, t\right)\right|_{C^{2+\beta-\alpha, \frac{2+\beta-\alpha}{2-\alpha}}\left(R_{T}^{N-1}\right)}=\left|u\left(x^{\prime}, 0, t\right)\right|_{C^{2+\beta-\alpha, \frac{2+\beta-\alpha}{2-\alpha}}\left(R_{T}^{N-1}\right)} \leq C|u|_{s, R_{+T}^{N}}^{(2+\gamma)} .
$$

Besides,

$$
\left|\nabla_{\left(x^{\prime}, x_{N}\right)} u\left(x^{\prime}, 0, t\right)\right|_{C^{1+\beta-\alpha, \frac{1+\beta-\alpha}{2-\alpha}}\left(R_{T}^{N-1}\right)} \leq C_{T}|u|_{s, R_{+T}^{N}}^{(2+\gamma)} .
$$


Proof. It follows directly from the definition of the space $C^{2+\gamma, \frac{2+\gamma}{2}}\left(R_{+T}^{N}\right)$ in (1.18) and from Lemma 2.1 that $u_{t}\left(x^{\prime}, 0, t\right) \in C^{\beta, \gamma / 2}\left(R_{T}^{N-1}\right)$. Therefore, $v_{t}\left(x^{\prime}, 0\right)=u_{t}\left(x^{\prime}, 0, t\right) \in C^{\beta, \gamma / 2}\left(R_{T}^{N-1}\right)$ and in addition

$$
\left|v_{t}\right|_{C^{\beta, \gamma / 2}\left(R_{T}^{N-1}\right)} \leq C|u|_{s, R_{+T}^{N}}^{(2+\gamma)} .
$$

Therefore, in view of (1.20) (see [45]) it is sufficient to prove uniformly in $t$ the following estimate

$$
|v(\cdot, t)|_{R^{N-1}}^{(2+\beta-\alpha)} \leq C|u|_{s, R_{+T}^{N}}^{(2+\gamma)}
$$

And for this it is sufficient to prove that uniformly in $t$ and in $x_{N}$ we have for all $i=\overline{1, N}$ for the function $w=u_{x_{i}}$

$$
\langle w\rangle_{x^{\prime}, R^{N-1}}^{(1+\beta-\alpha)} \leq C|u|_{s, R_{+T}^{N}}^{(2+\gamma)}
$$

So let $w=u_{x_{i}}, i=\overline{1, N}$. From (2.5) (see [54]) it follows that to prove (2.33) it is sufficient to show that for arbitrary $h>0$ the following inequality holds

$$
\frac{\left|\Delta_{h, x^{\prime}}^{2} w\left(x_{N}\right)\right|}{h^{1+\beta-\alpha}} \leq C|u|_{s, R_{+T}^{N}}^{(2+\gamma)} .
$$

Here $\Delta_{h, x^{\prime}}^{2} w\left(x_{N}\right) \equiv \Delta_{h, x^{\prime}}^{2} w \equiv \Delta_{h, x^{\prime}}^{2} w\left(x^{\prime}, x_{N}, t\right)$ is the second difference from the function $w$ with respect to the variable $x_{j}^{\prime}, j=\overline{1, N-1}$, with the step $h$, that is,

$$
\Delta_{h, x^{\prime}}^{2} w\left(x_{N}\right)=u\left(x^{\prime}+\vec{j} h, x_{N}, t\right)-2 u\left(x^{\prime}, x_{N}, t\right)+u\left(x^{\prime}-\vec{j} h, x_{N}, t\right) .
$$

Consider the following two cases. Let first

$$
h \leq x_{N} .
$$

Then according to the mean value theorem we have with some $\theta_{1}, \theta_{2} \in(0,1)$

$$
\begin{aligned}
\frac{\left|\Delta_{h, x^{\prime}}^{2} w\left(x_{N}\right)\right|}{h^{1+\beta-\alpha}} & \leq \frac{\left|x_{N}^{\alpha} \Delta_{h, x^{\prime}}^{2} w\left(x_{N}\right)\right|}{h^{1+\beta}} \\
& =\left|x_{N}^{\alpha} \frac{w_{x_{j}}\left(x^{\prime}+\vec{j} \theta_{1} h, x_{N}, t\right)-w_{x_{j}}\left(x^{\prime}-\vec{j} \theta_{2} h, x_{N}, t\right) \mid}{h^{\beta}}\right| \\
& =\frac{\left|x_{N}^{\alpha} u_{x_{i} x_{j}}\left(x^{\prime}+\vec{j} \theta_{1} h, x_{N}, t\right)-x_{N}^{\alpha} u_{x_{i} x_{j}}\left(x^{\prime}+\vec{j} \theta_{2} h, x_{N}, t\right)\right|}{h^{\beta}} \\
& \leq C\left\langle x_{N}^{\alpha} u_{x_{i} x_{j}}\right\rangle_{x^{\prime}, R_{+T}^{N}}^{(\beta)} \leq C|u|_{s, R_{+T}^{N}}^{(2+\gamma)} .
\end{aligned}
$$

Let now $h \geq x_{N}$. Write the difference $\Delta_{h, x^{\prime}}^{2} w\left(x_{N}\right)$ in the form

$$
\begin{aligned}
\Delta_{h, x^{\prime}}^{2} w\left(x_{N}\right)= & -\Delta_{h, x^{\prime}}^{2}\left(w\left(x^{\prime}, x_{N}+h, t\right)-w\left(x^{\prime}, x_{N}+h, t\right)\right) \\
& +\Delta_{h, x^{\prime}}^{2} w\left(x^{\prime}, x_{N}+h, t\right) \equiv A_{1}+A_{2} .
\end{aligned}
$$


In view of the fact that for the expression $A_{2}$ condition (2.36) holds, that is $h \leq x_{N}+h$, completely analogous to $(2.37)$,

$$
\frac{\left|A_{2}\right|}{h^{1+\beta-\alpha}} \leq C|u|_{s, R_{+T}^{N}}^{(2+\gamma)} .
$$

To estimate the expression $\left|A_{1}\right| / h^{1+\beta-\alpha}$ we use the formula

$$
w\left(x^{\prime}, x_{N}+h, t\right)-w\left(x^{\prime}, x_{N}, t\right)=h \int_{0}^{1} w_{x_{N}}\left(x^{\prime}, x_{N}+\theta h, t\right) d \theta .
$$

Consequently, in view of $w_{x_{N}}=u_{x_{i} x_{N}}$,

$$
\begin{aligned}
\frac{\left|A_{1}\right|}{h^{1+\beta-\alpha}} & \leq \int_{0}^{1} \frac{h^{\alpha}}{\left(x_{N}+\theta h\right)^{\alpha}}\left|\frac{\Delta_{h, x^{\prime}}^{2}\left(x_{N}+\theta h\right)^{\alpha} u_{x_{i} x_{N}}\left(x^{\prime}, x_{N}+\theta h, t\right)}{h^{\beta}}\right| d \theta \\
& \leq C\left\langle x_{N}^{\alpha} u_{x_{i} x_{N}}\right\rangle_{x^{\prime}, R_{+T}^{N}}^{(\beta)} \int_{0}^{1}\left(x_{N} / h+\theta\right)^{-\alpha} d \theta \leq C|u|_{s, R_{+T}^{N}}^{(2+\gamma)} .
\end{aligned}
$$

Thus, from (2.39) and (2.40) we obtain (2.34), and so we have also (2.33) and (2.32). Together with (2.31) this completes the proof of (2.29).

We now show inequality (2.30). Note that for tangential derivatives $u_{x_{i}}$, $i=\overline{1, N-1}$ this inequality follows from above estimate (2.29) and from [45], (1.20). However, for $u_{x_{N}}$ we need a distinct proof. We show (2.30) for $u_{x_{k}}$, $k=\overline{1, N}$.

According to (2.5) it is enough to show that for $h>0$

$$
\left|\Delta_{h, t}^{2} u_{x_{k}}(x, t)\right| \leq C|u|_{s, R_{+T}^{N}}^{(2+\gamma)} h^{\frac{1+\beta-\alpha}{2-\alpha}},
$$

where

$$
\Delta_{h, t}^{2} v(x, t)=\Delta_{h, t}^{2} v=v(x, t+2 h)-2 v(x, t+h)+v(x, t)
$$

is the second difference of a function $v$ with respect to the variable $t$ with the step $h$.

Let first

$$
h^{\frac{1}{2-\alpha}} \leq x_{N}
$$

Then we use the following interpolation inequality

$$
\left|v_{x_{k}}\right|_{\Pi_{T}\left(x_{N}\right)}^{(0)} \leq C\left(|v|_{\Pi_{T}\left(x_{N}\right)}^{(0)}\right)^{1 / 2}\left(\langle v\rangle_{\Pi_{T}\left(x_{N}\right)}^{(2)}\right)^{1 / 2}
$$

Here we denote $\Pi_{T}\left(x_{N}\right)=\left\{(y, t): x_{N} / 2<y_{N}<3 x_{N} / 2,0<t<T\right\}$, and for an integer $k$

$$
\langle v\rangle_{\bar{\Omega}}^{(k)}=\sum_{|\omega|=k}\left|D_{x}^{\omega} v\right|_{\frac{\Omega}{\Omega}}^{(0)} .
$$

Note that the constant $C$ in (2.43) does not depend on $x_{N}$ as it follows from scaling arguments. Inequality (2.43) easily follows from the well-known interpolation inequality (see, for example, [45], [50], Ch.1)

$$
\langle v\rangle_{\Pi_{T}\left(x_{N}\right)}^{(1)} \leq C\left(|v|_{\Pi_{T}\left(x_{N}\right)}^{(0)}\right)^{1 / 2}\left(|v|_{\Pi_{T}\left(x_{N}\right)}^{(2)}\right)^{1 / 2}
$$


where for an integer $k$

$$
|v|_{\Omega}^{(k)}=\sum_{|\omega| \leq k}\left|D_{x}^{\omega} v\right|_{\frac{(0)}{\Omega}}^{(0 .}
$$

To obtain (2.43) from (2.44) it is enough to apply first to the right-hand side of (2.44) the Young inequality with $\varepsilon>0$ and get

$$
\langle v\rangle_{\Pi_{T}\left(x_{N}\right)}^{(1)} \leq \varepsilon\left(|v|_{\Pi_{T}\left(x_{N}\right)}^{(0)}+\langle v\rangle_{\Pi_{T}\left(x_{N}\right)}^{(1)}+\langle v\rangle_{\Pi_{T}\left(x_{N}\right)}^{(2)}\right)+C \frac{1}{\varepsilon}|v|_{\Pi_{T}\left(x_{N}\right)}^{(0)} .
$$

From this inequality it follows that for $\varepsilon<1 / 2$ and with some another constant C

$$
\langle v\rangle_{\Pi_{T}\left(x_{N}\right)}^{(1)} \leq \varepsilon\langle v\rangle_{\Pi_{T}\left(x_{N}\right)}^{(2)}+C \frac{1}{\varepsilon}|v|_{\Pi_{T}\left(x_{N}\right)}^{(0)} .
$$

Optimising this inequality with respect to $\varepsilon$ in usual way we get (2.43).

From (2.43) we obtain for $\Delta_{h, t}^{2} u_{x_{k}}(x, t)$

$$
\left|\Delta_{h, t}^{2} u_{x_{k}}(x, t)\right| \leq C\left(\left|\Delta_{h, t}^{2} u\right|_{\Pi_{T}\left(x_{N}\right)}^{(0)}\right)^{1 / 2}\left(\left|\Delta_{h, t}^{2} u\right|_{\Pi_{T}\left(x_{N}\right)}^{(2)}\right)^{1 / 2} .
$$

In view of $(2.5)$ and the properties of the space $C_{s}^{2+\gamma, \frac{2+\gamma}{2}}\left(R_{+T}^{N}\right)$ (see $(1.20)$, $[45,54])$ we have

$$
\begin{gathered}
\left|\Delta_{h, t}^{2} u\right|_{\Pi_{T}\left(x_{N}\right)}^{(0)} \leq C|u|_{s, R_{+T}^{N}}^{(2+\gamma)} h^{\frac{2+\gamma}{2}} \\
\left|\Delta_{h, t}^{2} u\right|_{\Pi_{T}\left(x_{N}\right)}^{(2)} \leq C|u|_{s, R_{+T}^{N}}^{(2+\gamma)} x_{N}^{-\alpha} h^{\frac{\gamma}{2}} .
\end{gathered}
$$

From (2.46) and (2.47), in view of (2.42), we obtain

$$
\left|\Delta_{h, t}^{2} u_{x_{k}}(x, t)\right| \leq C|u|_{s, R_{+T}^{N}}^{(2+\gamma)} h^{\frac{2+\gamma}{4}+\frac{\gamma}{4}-\frac{\alpha}{2(2-\alpha)}}=C|u|_{s, R_{+T}^{N}}^{(2+\gamma)} h^{\frac{1+\beta-\alpha}{(2-\alpha)}},
$$

that is inequality $(2.41)$.

Let now

$$
h^{\frac{1}{2-\alpha}} \geq x_{N}
$$

Write $\Delta_{h, t}^{2} u_{x_{k}}(x, t)$ as

$$
\begin{aligned}
\Delta_{h, t}^{2} u_{x_{k}}(x, t)= & -\Delta_{h, t}^{2}\left[u_{x_{k}}\left(x^{\prime}, x_{N}+h^{\frac{1}{2-\alpha}}, t\right)-u_{x_{k}}(x, t)\right] \\
& +\Delta_{h, t}^{2} u_{x_{k}}\left(x^{\prime}, x_{N}+h^{\frac{1}{2-\alpha}}, t\right) \equiv A_{1}+A_{2},
\end{aligned}
$$

and for $A_{2}$, by the above case (2.42), the estimate

$$
\left|A_{2}\right| \leq C|u|_{s, R_{+T}^{N}}^{(2+\gamma)} h^{\frac{1+\beta-\alpha}{(2-\alpha)}}
$$

is valid.

To estimate the expression $A_{1}$ write it as

$$
A_{1}=-h^{\frac{1}{2-\alpha}} \int_{0}^{1} \Delta_{h, t}^{2} u_{x_{k} x_{N}}\left(x^{\prime}, x_{N}+\theta h^{\frac{1}{2-\alpha}}, t\right) d \theta .
$$


Thus, we have for $A_{1}$

$$
\begin{aligned}
\left|A_{1}\right| & \leq C|u|_{s, R_{+T}^{N}}^{(2+\gamma)} h^{\frac{1}{2-\alpha}} h^{\frac{\gamma}{2}} \int_{0}^{1}\left(x_{N}+\theta h^{\frac{1}{2-\alpha}}\right)^{-\alpha} d \theta \\
& \leq C|u|_{s, R_{+T}^{N}}^{(2+\gamma)} h^{\frac{1}{2-\alpha}+\frac{\gamma}{2}-\frac{\alpha}{2-\alpha}} \int_{0}^{1} \theta^{-\alpha} d \theta=C|u|_{s, R_{+T}^{N}}^{(2+\gamma)} h^{\frac{1+\beta-\alpha}{(2-\alpha)}}
\end{aligned}
$$

that is, again inequality (2.41).

The lemma is proved.

Thus, due to the possibility of the local straightening of the boundary, the following is true.

Lemma 2.3. Let functions $u^{ \pm}(x, t)$ belong to the spaces $C_{s}^{2+\gamma, \frac{2+\gamma}{2}}\left(\bar{\Omega}_{T}^{ \pm}\right)$. Then the functions $v^{ \pm}(x, t)=\left.u^{ \pm}(x, t)\right|_{x \in \Gamma}$ belong to the space $C^{2+\beta-\alpha, \frac{2+\beta-\alpha}{2-\alpha}}\left(\Gamma_{T}\right)$, and

$$
\left|v^{ \pm}\right|_{C^{2+\beta-\alpha, \frac{2+\beta-\alpha}{2-\alpha}}\left(\Gamma_{T}\right)} \leq C|u|_{s, \bar{\Omega}_{T}^{ \pm}}^{(2+\gamma)}
$$

Besides,

$$
\left.\left|\nabla u^{ \pm}\right|_{\Gamma_{T}}\right|_{C^{1+\beta-\alpha, \frac{1+\beta-\alpha}{2-\alpha}}\left(\Gamma_{T}\right)} \leq C\left|u^{ \pm}\right|_{s, \bar{\Omega}_{T}^{ \pm}}^{(2+\gamma)}
$$

\subsection{An extension from the surface $\Gamma_{T}$ of functions from the space $C^{2+\beta-\alpha, \frac{2+\beta-\alpha}{2-\alpha}}\left(\Gamma_{T}\right)$}

In this section we prove the converse of Lemma 2.3, that is, we show that any function from the class $C^{2+\beta-\alpha, \frac{2+\beta-\alpha}{2-\alpha}}\left(\Gamma_{T}\right)=C^{2+\beta-\alpha, 1+\gamma / 2}\left(\Gamma_{T}\right)$ can be extended to all domains $\bar{\Omega}_{T}^{ \pm}$up to functions of the class $C_{s}^{2+\gamma, \frac{2+\gamma}{2}}\left(\bar{\Omega}_{T}^{ \pm}\right)$, and the extension operator is bounded (here, as above $\beta=\gamma(1-\alpha / 2)$ ). Such an extension operator is constructed in the standard way by applying a sufficiently small partition of unity in the neighborhood of $\Gamma$ and by the local straightening of the boundary $\Gamma$, see [43]. In this case, it is enough to require the $C^{2+\gamma}$-smoothness of the boundary $\Gamma$. Therefore, the existence of the extension operator follows in the standard way from the following lemma.

Lemma 2.4. Let $f\left(x^{\prime}, t\right)$ be a function from the class $C^{2+\beta-\alpha, 1+\gamma / 2}\left(R_{T}^{N-1}\right)$ with finite support in $R_{+T}^{N}$ at $x_{N}=0$. Then $f$ can be extended to the domain $x_{N}>0$ up to a function $u(x, t)$ from the class $C_{s}^{2+\gamma, \frac{2+\gamma}{2}}\left(R_{+T}^{N}\right)$, and

$$
|u|_{s, R_{+T}^{N}}^{(2+\gamma)} \leq C|f|_{C^{2+\beta-\alpha, 1+\gamma / 2}\left(R_{T}^{N-1}\right)} .
$$

Proof. Let $u(x, t)$ be the solution of the following Dirichlet problem with the parameter $t \in[0, T]$ :

$$
\begin{gathered}
\Delta u=0, \quad x \in R_{+}^{N} \quad\left(x_{N}>0\right), \\
\left.u\right|_{x_{N}=0}=f\left(x^{\prime}, t\right), \\
u \rightarrow 0, \quad|x| \rightarrow \infty .
\end{gathered}
$$


As it is well known, the solution of $(2.54)-(2.56)$ is given by the potential of the double layer, defined by the Newton potential.

Note first, that for problem (2.54)-(2.56) we have the following maximum principle

$$
|u|_{R_{+}^{N}}^{(0)} \leq|f|_{R^{N-1}}^{(0)}
$$

Indeed, by (2.56) we can choose $K>0$ so large that $|u| \leq|f|_{R_{T}^{N-1}}^{(0)} / 2$ for $|x| \geq K$. By the properties of the double layer potential and the finiteness of $f$ a constant $K$ can be chosen independent of $t$. Now consider in the domain $B_{K}=R_{+}^{N} \cap\{|x|<K\}$ the functions $v^{ \pm}= \pm u+|f|_{R^{N-1}}^{(0)}$. Within this domain we have

$$
\Delta v^{ \pm}=0, \quad x \in B_{K},
$$

and on the boundary $\partial B_{K}=\left\{x_{N}=0,\left|x^{\prime}\right| \leq K\right\} \cup\left\{x_{N}>0,\left|x^{\prime}\right|=K\right\}$ the inequality

$$
v^{ \pm} \geq 0, \quad x \in \partial B_{K}
$$

holds. It follows from (2.58), (2.59) and from the maximum principle that $v^{ \pm} \geq 0$ in $\bar{B}_{K}$ for all $t$ and thus,

$$
|u|_{B_{K}}^{(0)} \leq|f|_{R^{N-1}}^{(0)} \quad t \in[0, T] .
$$

Due to the choice of $K$ we have inequality (2.57) on the whole domain $R_{+T}^{N}$.

Consider first the properties of the function $u(x, t)$ with respect to $t$. Let $v(x, t)$ be the solution of $(2.54)-(2.56)$ with the boundary condition

$$
\left.v\right|_{x_{N}=0}=f_{t}\left(x^{\prime}, t\right)
$$

instead of (2.55). Consider also for $h>0$ the following function

$$
u_{h}(x, t)=\frac{u(x, t+h)-u(x, t)}{h},
$$

which satisfies problem (2.54)-(2.56) with the boundary condition

$$
\left.u_{h}\right|_{x_{N}=0}=f_{h}\left(x^{\prime}, t\right) \equiv \frac{f\left(x^{\prime}, t+h\right)-f\left(x^{\prime}, t\right)}{h} .
$$

Let further $w(x, t)=u_{h}(x, t)-v(x, t)$ and the function $w(x, t)$ also satisfies problem (2.54)-(2.56) but with the boundary condition

$$
\left.w\right|_{x_{N}=0}=f_{h}\left(x^{\prime}, t\right)-f_{t}\left(x^{\prime}, t\right) \equiv \varphi_{h}\left(x^{\prime}, t\right) .
$$

Due to the properties of the function $f\left(x^{\prime}, t\right)$ we have with some $\theta\left(x^{\prime}, t, h\right) \in$ $(0,1)$ according to the mean value theorem

$$
\begin{aligned}
\left|\varphi_{h}\left(x^{\prime}, t\right)\right| & =\left|f_{h}\left(x^{\prime}, t\right)-f_{t}\left(x^{\prime}, t\right)\right| \\
& =\left|f_{t}\left(x^{\prime}, t+\theta h\right)-f_{t}\left(x^{\prime}, t\right)\right| \leq\left\langle f_{t}\left(x^{\prime}, t\right)\right\rangle_{t, R_{T}^{N-1}}^{(\gamma / 2)} h^{\gamma / 2} \rightarrow 0, h \rightarrow 0 .
\end{aligned}
$$

Consequently, on the base of (2.57),

$$
\left|u_{h}-v\right|_{R_{+T}^{N}}^{(0)} \leq C h^{\gamma / 2} \rightarrow 0, \quad h \rightarrow 0
$$


This means that the function $u(x, t)$ has the derivative with respect to the variable $t$ for $x \in R_{+T}^{N}$ and $u_{t}(x, t)=v(x, t)$, that is $u_{t}(x, t)$ satisfies problem (2.54)-(2.56) with boundary condition (2.60).

Further, considering the function

$$
v_{h}(x, t)=\frac{u_{t}(x, t+h)-u_{t}(x, t)}{h^{\gamma / 2}},
$$

we see that it satisfies the same problem with the boundary condition

$$
\left.v_{h}(x, t)\right|_{x_{N}=0}=\frac{f_{t}\left(x^{\prime}, t+h\right)-f_{t}\left(x^{\prime}, t\right)}{h^{\gamma / 2}} \equiv f_{t h}\left(x^{\prime}, t\right) .
$$

Thus, on the base of (2.57) again

$$
\left|\frac{u_{t}(x, t+h)-u_{t}(x, t)}{h^{\gamma / 2}}\right|_{R_{+T}^{N}}^{(0)} \leq C\left|\frac{f_{t}\left(x^{\prime}, t+h\right)-f_{t}\left(x^{\prime}, t\right)}{h^{\gamma / 2}}\right|_{R_{T}^{N-1}}^{(0)} .
$$

As $h$ is arbitrary this means that

$$
\left\langle u_{t}(x, t)\right\rangle_{t, R_{+T}^{N}}^{(\gamma / 2)} \leq C\left\langle f_{t}\left(x^{\prime}, t\right)\right\rangle_{t, R_{T}^{N-1}}^{(\gamma / 2)}
$$

Consider now the properties of the function $u(x, t)$ with respect to the variables $x$. First, it follows from the results of $[56,57]$ that for each $t \in[0, T]$ we have $u \in C_{x}^{2+\beta-\alpha}\left(R_{+T}^{N}\right)$ due to $f \in C_{x}^{2+\beta-\alpha}\left(R_{T}^{N-1}\right)$ and

$$
|u|_{C_{x}^{2+\beta-\alpha}\left(R_{+T}^{N}\right)} \leq C|f|_{C_{x}^{2+\beta-\alpha}\left(R_{T}^{N-1}\right)} .
$$

Here the symbol $x$ at the bottom of the space notation means that we consider the smoothness only with respect to $x$ and

$$
\begin{aligned}
|u|_{C_{x}^{2+\beta-\alpha}\left(R_{+T}^{N}\right)} & =\max _{t \in[0, T]}|u(\cdot, t)|_{C^{2+\beta-\alpha}\left(R_{+}^{N}\right)}, \\
|f|_{C_{x}^{2+\beta-\alpha}\left(R_{T}^{N-1}\right)} & =\max _{t \in[0, T]}|f(\cdot, t)|_{C^{2+\beta-\alpha}\left(R^{N-1}\right)} .
\end{aligned}
$$

Show that the following estimates

$$
\begin{gathered}
\left\langle x_{N}^{\alpha} D^{2} u\right\rangle_{x, R_{+T}^{N}}^{(\beta)} \leq C|f|_{C_{x}^{2+\beta-\alpha}\left(R_{T}^{N-1}\right)}, \\
\left\langle\widetilde{x}_{N}^{\gamma \alpha / 2}\left(x_{N}^{\alpha} D^{2} u\right)\right\rangle_{x, R_{+T}^{N}}^{(\gamma)} \leq C|f|_{C_{x}^{2+\beta-\alpha}\left(R_{T}^{N-1}\right)}
\end{gathered}
$$

are valid, that is,

$$
H_{\alpha}^{\gamma}\left(x_{N}^{\alpha} D^{2} u\right) \leq C|f|_{C_{x}^{2+\beta-\alpha}\left(R_{T}^{N-1}\right)} .
$$

We will use the fact that, as it follows from [58], Chapter 5.4, the condition $f \in C_{x}^{l}\left(R^{N-1}\right)$ in $(2.55), l \in(0,2)$, is equivalent to the condition

$$
\left|D_{x}^{k} u\right| \leq C_{k} x_{N}^{-k+l}|f|_{C_{x}^{l}\left(R^{N-1}\right)}, \quad k \geq 2,
$$

where here and below $D_{x}^{k} u=D^{k} u$ means a derivative of the $k$-th order with respect to $x$ of the function $u(x, t)$. Since it is important to prove (2.69) for $x_{N}<1$ only (for $x_{N}>1$, such the estimate follows from the local estimates 
and is well- known), we consider only the case $x_{N}<1$. We also use the wellknown interpolation inequality

$$
\langle v(x)\rangle_{x, \bar{\Omega}}^{(\beta)} \leq C\left(|v|_{\frac{(0)}{\Omega}}^{(1-\beta}\left(\langle v\rangle_{\bar{\Omega}}^{(1)}\right)^{\beta},\right.
$$

which is valid for functions $v(x) \in C^{1}(\bar{\Omega})$, where $\Omega$ is a domain (possibly unbounded) with sufficiently smooth boundary (see, for example [50] Chapter 1 ). This inequality is analogous to (2.43) and it is important that the constant $C$ does not depend on the size of the domain $\Omega$ under scaling. In addition, at the proof of (2.69) without loss of generality we prove smoothness of the function $x_{N}^{\alpha} D^{2} u$ with respect to $x^{\prime}$ and with respect to $x_{N}$ separately. So we obtain estimate (2.69) separately for these two cases.

Let first $x_{N}$ be fixed. Then by (2.73) and (2.72),

$$
\begin{aligned}
& \left\langle x_{N}^{\alpha} D^{2} u\left(\cdot, x_{N}\right)\right\rangle_{x^{\prime}, R^{N-1}}^{(\beta)} \leq C\left(\left|x_{N}^{\alpha} D^{2} u\right|_{x^{\prime}, R^{N-1}}^{(0)}\right)^{1-\beta}\left(\left|\nabla_{x^{\prime}} x_{N}^{\alpha} D^{2} u\right|_{x^{\prime}, R^{N-1}}^{(0)}\right)^{\beta} \\
& \quad \leq C|f|_{C_{x}^{2+\beta-\alpha}\left(R_{T}^{N-1}\right)}\left(x_{N}^{\alpha} x_{N}^{-2+(2+\beta-\alpha)}\right)^{1-\beta}\left(x_{N}^{\alpha} x_{N}^{-3+(2+\beta-\alpha)}\right)^{\beta} \\
& \quad=C|f|_{C_{x}^{2+\beta-\alpha}\left(R_{T}^{N-1}\right)}
\end{aligned}
$$

that is, estimate (2.69) with respect to $x^{\prime}$.

Analogously, using (2.73) and (2.72), we prove (2.70) with respect to $x^{\prime}$ :

$$
\begin{aligned}
& x_{N}^{\gamma \alpha / 2}\left\langle x_{N}^{\alpha} D^{2} u\left(\cdot, x_{N}\right)\right\rangle_{x^{\prime}, R^{N-1}}^{(\gamma)} \\
& \quad \leq C x_{N}^{\gamma \alpha / 2}\left(\left|x_{N}^{\alpha} D^{2} u\right|_{x^{\prime}, R^{N-1}}^{(0)}\right)^{1-\gamma}\left(\left|x_{N}^{\alpha} D^{2} u\right|_{x^{\prime}, R^{N-1}}^{(1)}\right)^{\gamma} \\
& \quad \leq C|f|_{C_{x}^{2+\beta-\alpha}\left(R_{T}^{N-1}\right)^{2} x_{N}^{\gamma \alpha / 2}\left(x_{N}^{\alpha} x_{N}^{-2+(2+\beta-\alpha)}\right)^{1-\gamma}\left(x_{N}^{\alpha} x_{N}^{-3+(2+\beta-\alpha)}\right)^{\gamma}} \\
& \quad=C|f|_{C_{x}^{2+\beta-\alpha}\left(R_{T}^{N-1}\right)} x_{N}^{\gamma \alpha / 2+\beta-\gamma}=C|f|_{C_{x}^{2+\beta-\alpha}\left(R_{T}^{N-1}\right)^{N-1} .}
\end{aligned}
$$

We prove now relations $(2.69),(2.70)$ with respect to the variable $x_{N}$. For this we fix some $\varepsilon_{0} \in(0,1 / 16)$ and consider the two cases, assuming without loss of generality that $\bar{x}_{N} \leq x_{N}$.

Let first

$$
\left|x_{N}-\bar{x}_{N}\right|=\left(x_{N}-\bar{x}_{N}\right) \geq \varepsilon_{0} x_{N}
$$

Then

$$
\frac{\left|x_{N}^{\alpha} D^{2} u(x, t)-\bar{x}_{N}^{\alpha} D^{2} u(\bar{x}, t)\right|}{\left|x_{N}-\bar{x}_{N}\right|^{\beta}} \leq C\left(\left|x_{N}^{\alpha-\beta} D^{2} u(x, t)\right|+\left|\bar{x}_{N}^{\alpha-\beta} D^{2} u(\bar{x}, t)\right|\right) .
$$

In this case, as above

$$
\left|x_{N}^{\alpha-\beta} D^{2} u(x, t)\right| \leq C|f|_{C_{x}^{2+\beta-\alpha}\left(R_{T}^{N-1}\right)} x_{N}^{\alpha-\beta} x_{N}^{-2+(2+\beta-\alpha)}=C|f|_{C_{x}^{2+\beta-\alpha}\left(R_{T}^{N-1}\right)},
$$


and similarly for $\left|\bar{x}_{N}^{\alpha-\beta} D^{2} u(\bar{x}, t)\right|$. In the same way

$$
x_{N}^{\gamma \alpha / 2} \frac{\left|x_{N}^{\alpha} D^{2} u(x, t)-\bar{x}_{N}^{\alpha} D^{2} u(\bar{x}, t)\right|}{\left|x_{N}-\bar{x}_{N}\right|^{\gamma}} \leq C\left(\left|x_{N}^{\alpha-\beta} D^{2} u(x, t)\right|+\left|\bar{x}_{N}^{\alpha-\beta} D^{2} u(\bar{x}, t)\right|\right)
$$

and then proceeding as in (2.78).

Let now

$$
0<\left(x_{N}-\bar{x}_{N}\right) \leq \varepsilon_{0} x_{N}
$$

and let also

$$
\begin{gathered}
\Pi\left(x_{N}\right)=\left\{y \in R_{+}^{N}: x_{N}-2 \varepsilon_{0} x_{N} \leq y_{N} \leq x_{N}+2 \varepsilon_{0} x_{N}\right\}, \\
\Pi_{T}\left(x_{N}\right)=\Pi\left(x_{N}\right) \times[0, T] .
\end{gathered}
$$

Then, taking into account that on $\Pi_{T}\left(x_{N}\right)$ we have $y_{N} \sim x_{N}$, as in the previous case

$$
\begin{aligned}
& \frac{\left|x_{N}^{\alpha} D^{2} u(x, t)-\bar{x}_{N}^{\alpha} D^{2} u(\bar{x}, t)\right|}{\left|x_{N}-\bar{x}_{N}\right|^{\beta}} \leq\left\langle y_{N}^{\alpha} D^{2} u(y, t)\right\rangle_{y, \Pi_{T}\left(x_{N}\right)}^{(\beta)} \\
& \quad \leq C\left(\left|y_{N}^{\alpha-\beta} D^{2} u\right|_{\Pi_{T}\left(x_{N}\right)}^{(0)}+x_{N}^{\alpha}\left\langle D^{2} u(y, t)\right\rangle_{y, \Pi_{T}\left(x_{N}\right)}^{(\beta)}\right) \equiv A_{1}+A_{2}
\end{aligned}
$$

Here $A_{1}$ is estimated in the same way as in (2.78), and $A_{2}$ - as well as in (2.74). This gives

$$
\frac{\left|x_{N}^{\alpha} D^{2} u(x, t)-\bar{x}_{N}^{\alpha} D^{2} u(\bar{x}, t)\right|}{\left|x_{N}-\bar{x}_{N}\right|^{\beta}} \leq C|f|_{C_{x}^{2+\beta-\alpha}\left(R_{T}^{N-1}\right)} .
$$

The estimate

$$
x_{N}^{\gamma \alpha / 2} \frac{\left|x_{N}^{\alpha} D^{2} u(x, t)-\bar{x}_{N}^{\alpha} D^{2} u(\bar{x}, t)\right|}{\left|x_{N}-\bar{x}_{N}\right|^{\gamma}} \leq C|f|_{C_{x}^{2+\beta-\alpha}\left(R_{T}^{N-1}\right)}
$$

is quite similar.

This completes the proof of (2.71).

Similarly, we obtain the properties with respect to the variables $x$ of the derivative $u_{t}$, that is,

$$
H_{\alpha}^{\gamma}\left(u_{t}\right) \leq C|f|_{C_{x}^{2+\beta-\alpha}\left(R_{T}^{N-1}\right)}
$$

because $u_{t}$ satisfies problem (2.54)-(2.56) with boundary condition (2.60). Indeed, since $\left.u_{t}\right|_{x_{N}=0}=f_{t}$,

$$
\left\langle u_{t}\right\rangle_{x, R_{+T}^{N}}^{(\beta)} \leq C\left\langle f_{t}\right\rangle_{x, R_{T}^{N-1}}^{(\beta)} \leq C|f|_{C^{2+\beta-\alpha, \frac{2+\beta-\alpha}{2-\alpha}}\left(R_{T}^{N-1}\right)} .
$$

Further, for $x, \bar{x} \in R_{+}^{N}, x_{N} \geq \bar{x}_{N}$ consider the difference

$$
\Delta(x, \bar{x}) u_{t}=x_{N}^{\gamma \alpha / 2} \frac{\left|u_{t}(x, t)-u_{t}(\bar{x}, t)\right|}{|x-\bar{x}|^{\gamma}} .
$$


If $|x-\bar{x}| \geq \varepsilon_{0} x_{N}$, then

$$
\begin{aligned}
\Delta(x, \bar{x}) u_{t} & \leq C \frac{x_{N}^{\gamma \alpha / 2}}{x_{N}^{\gamma \alpha / 2}} \frac{\left|u_{t}(x, t)-u_{t}(\bar{x}, t)\right|}{|x-\bar{x}|^{\beta}} \leq C\left\langle u_{t}\right\rangle_{x, R_{+T}^{N}}^{(\beta)} \\
& \leq C|f|_{C^{2+\beta-\alpha, \frac{2+\beta-\alpha}{2-\alpha}}\left(R_{T}^{N-1}\right)}
\end{aligned}
$$

If now $|x-\bar{x}| \leq \varepsilon_{0} x_{N}$, then $x_{N} \sim \bar{x}_{N}$ and using (2.72) we obtain

$$
\begin{aligned}
\Delta(x, \bar{x}) u_{t} & \leq C x_{N}^{\gamma \alpha / 2} \frac{\left|u_{t}(x, t)-u_{t}(\bar{x}, t)\right|}{|x-\bar{x}|}|x-\bar{x}|^{1-\gamma} \\
& \leq C x_{N}^{\gamma \alpha / 2}\left|\nabla_{x} u_{t}(x, t)\right|_{\Pi_{T}\left(x_{N}\right)}^{(0)} x_{N}^{1-\gamma} \\
& \leq C\left|f_{t}\right|_{C_{x}^{\beta}\left(R_{T}^{N-1}\right)} x_{N}^{\gamma \alpha / 2+1-\gamma} x_{N}^{-1+\beta} \\
& =C\left|f_{t}\right|_{C_{x}^{\beta}\left(R_{T}^{N-1}\right)} .
\end{aligned}
$$

Now (2.85) follows from (2.88) and (2.89).

Let us show now the smoothness of the function $x_{N}^{\alpha} D^{2} u(x, t)$ with respect to the variable $t$, that is show that

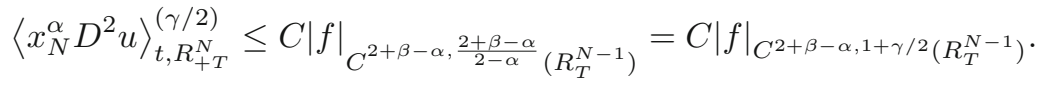

For this we fix some $h>0$ and consider the function

$$
v_{h}(x, t)=\frac{u(x, t+h)-u(x, t)}{h^{\gamma / 2}},
$$

which satisfies problem (2.54)-(2.56) with the following boundary condition

$$
\left.v_{h}(x, t)\right|_{x_{N}=0}=\varphi_{h}(x, t) \equiv \frac{f(x, t+h)-f(x, t)}{h^{\gamma / 2}} .
$$

It follows from the results of [45], (1.20) that $\varphi_{h}(x, t) \in C_{x}^{2-\alpha}\left(R^{N-1}\right)$ with respect to the variables $x$ uniformly with respect to the variable $t$ and

$$
\max _{t \in[0, T]}\left|\varphi_{h}(\cdot, t)\right|_{R^{N-1}}^{(2-\alpha)} \leq C|f|_{C^{2+\beta-\alpha, 1+\gamma / 2}\left(R_{T}^{N-1}\right)} .
$$

Now note that

$$
\frac{x_{N}^{\alpha} D^{2} u(x, t+h)-x_{N}^{\alpha} D^{2} u(x, t)}{h^{\gamma / 2}}=x_{N}^{\alpha} D^{2} v_{h}(x, t) .
$$

Consequently, it follows from (2.72) that

$$
\left|x_{N}^{\alpha} D^{2} v_{h}(x, t)\right| \leq C x_{N}^{\alpha} \max _{t \in[0, T]}\left|\varphi_{h}(\cdot, t)\right|_{R^{N-1}}^{(2-\alpha)} x_{N}^{-2+(2-\alpha)}=C \max _{t \in[0, T]}\left|\varphi_{h}(\cdot, t)\right|_{R^{N-1}}^{(2-\alpha)} .
$$

So (2.90) follows from (2.94) and (2.93) in view of the definition of $v(x, t)$.

Multiplying now the function $u(x, t)$ by a smooth cut-of function $\eta(x)$ with finite support that is equal to one in a neighborhood of support of $f\left(x^{\prime}, t\right)$, we get an extension with finite support of $f\left(x^{\prime}, t\right)$ of desired class and the estimate (2.53) is valid.

Lemma 2.4 is proved. 
From this lemma in the standard way (see [43]) as it was described in the beginning of this section we get the following assertion.

Lemma 2.5. There exist bounded extension operators $E^{+}$and $E^{-}$, such that

$$
\begin{gathered}
\rho \in C^{2+\beta-\alpha, \frac{2+\beta-\alpha}{2-\alpha}}\left(\Gamma_{T}\right) \rightarrow E \rho \equiv E^{ \pm} \rho \equiv \rho^{ \pm} \in C_{s}^{2+\gamma, \frac{2+\gamma}{2}}\left(\bar{\Omega}_{T}^{ \pm}\right), \\
\left|E^{ \pm} \rho\right|_{C_{s}^{2+\gamma, \frac{2+\gamma}{2}}}\left(\bar{\Omega}_{T}^{ \pm}\right) \\
\leq C|\rho|_{C^{2+\beta-\alpha, \frac{2+\beta-\alpha}{2-\alpha}}}\left(\Gamma_{T}\right)
\end{gathered}
$$

and we can assume that the supports of the extended functions $\rho^{ \pm}$are included in the sufficiently small neighbourhood $\mathcal{N}_{T}$ of the surface $\Gamma_{T}$.

We will denote the extended functions $\rho \equiv E \rho \equiv E^{ \pm} \rho$ by the same symbol $\rho$ to not to overload the notation, that is,

$$
\left.\left.\rho\right|_{\bar{\Omega}_{T}^{ \pm}} \equiv \rho^{ \pm} \equiv E^{ \pm} \rho \equiv E \rho\right|_{\Omega_{T}^{ \pm}} \cdot
$$

Due to Lemma 2.4 and to the results of [42] we have the following assertion.

Lemma 2.6. Let functions $f$ and $g$ are finite and

$$
f\left(x^{\prime}, t\right) \in C_{0}^{2+\beta-\alpha, \frac{2+\beta-\alpha}{2-\alpha}}\left(R_{T}^{N-1}\right), \quad g(x, t) \in C_{s .0}^{\gamma}\left(R_{+T}^{N}\right) .
$$

Then the problem

$$
\begin{gathered}
\frac{\partial u}{\partial t}-x_{N}^{\alpha} \Delta u=g(x, t), \quad(x, t) \in R_{+T}^{N}, \\
u\left(x^{\prime}, 0, t\right)=f\left(x^{\prime}, t\right), \quad x_{N}=0, t \in[0, T], \\
u(x, 0)=0, \quad x \in R_{+}^{N}
\end{gathered}
$$

has the unique solution $u(x, t)$, which satisfies the estimate

$$
|u|_{s, R_{+T}^{N}}^{(2+\gamma)} \leq C\left(|f|_{C^{2+\beta-\alpha, \frac{2+\beta-\alpha}{2-\alpha}}\left(R_{T}^{N-1}\right)}+|g|_{s, R_{+T}^{N}}^{(\gamma)}\right) .
$$

For the case $f=0$ this lemma was proved in [42] and general case is reduced to such case by Lemma 2.4 .

Consider now the initial-boundary value problem for the degenerate equations of the form

$$
\begin{gathered}
\frac{\partial u^{ \pm}}{\partial t}-\left(d^{ \pm}(x)\right)^{\alpha} B^{ \pm}(x, t) \Delta u^{ \pm}=g^{ \pm}(x, t), \quad(x, t) \in \Omega_{T}^{ \pm}, \\
\left.u^{ \pm}\right|_{\Gamma_{T}}=f^{ \pm}(x, t), \\
\left.u^{ \pm}\right|_{\Gamma_{T}^{ \pm}}=h^{ \pm}(x, t), \\
u^{ \pm}(x, 0)=0,
\end{gathered}
$$

where the functions $d^{ \pm}(x)$ are introduced in (1.11),

$$
\begin{gathered}
B^{ \pm}(x, t) \in C^{\gamma, \gamma / 2}\left(\bar{\Omega}_{T}^{ \pm}\right), \quad \nu \leq B^{ \pm} \leq \nu^{-1}, \\
g^{ \pm} \in C_{0, s}^{\gamma, \gamma / 2}\left(\bar{\Omega}_{T}^{ \pm}\right), f^{ \pm} \in C_{0}^{2+\beta-\alpha, \frac{2+\beta-\alpha}{2-\alpha}}\left(\Gamma_{T}\right), h^{ \pm} \in C_{0}^{2+\gamma, \frac{2+\gamma}{2}}\left(\Gamma_{T}^{ \pm}\right) .
\end{gathered}
$$


Theorem 2.7. Problem (2.102)-(2.105) has the unique solution from the space $C_{0, s}^{2+\gamma, \frac{2+\gamma}{2}}\left(\bar{\Omega}_{T}^{ \pm}\right)$and the following estimate is valid

$$
\left|u^{ \pm}\right|_{s, \bar{\Omega}_{T}^{ \pm}}^{(2+\gamma)} \leq C\left(\left|g^{ \pm}\right|_{s, \bar{\Omega}_{T}^{ \pm}}^{(\gamma)}+\left|f^{ \pm}\right|_{\Gamma_{T}}^{\left(2+\beta-\alpha, \frac{2+\beta-\alpha}{2-\alpha}\right)}+\left|h^{ \pm}\right|_{\Gamma_{T}^{ \pm}}^{\left(2+\gamma, \frac{2+\gamma}{2}\right)}\right) .
$$

This theorem was proved in [42] for the case $f^{ \pm}=0$ and from Lemma 2.5 we get this assertion for general case.

\section{A model problem with dynamic boundary conditions for degenerate equations}

In this section we consider the simplest problem in half-spaces, which corresponds to the linearization of the original nonlinear Stefan problem. This model problem is a parabolic problem with dynamic boundary conditions. It is convenient in this section to consider the domains with $t \geq-a$ as it will allow us to consider the points $(x, 0)$ with $t=0$ as interior points of a general position. This will facilitate the further notation and preserve the notation of paper [53].

Let $a \geq 0$ be a fixed number. Denote $Q_{ \pm}^{N}=\left\{(x, t): x \in R_{ \pm}^{N}, t \geq-a\right\}$, $Q^{N-1}=\left\{\left(x^{\prime}, t\right): x^{\prime} \in R^{N-1}, t \geq-a\right\}$. Denote further $R_{ \pm, T}^{N, a}=Q_{ \pm}^{N} \cap\{t \leq T\}$, $R_{T}^{N-1, a}=Q^{N-1} \cap\{t \leq T\}$. Similar to (1.21) we agree that zero at the bottom of the designation of functional spaces means the subspace of the corresponding space whose elements vanish at $t=-a$ together with all its derivatives with respect to $t$. Let a function $f\left(x^{\prime}, t\right)$ is defined in $Q^{N-1}$, has finite with respect to $x$ support, and let $f\left(x^{\prime}, t\right)$ is such that

$$
f\left(x^{\prime},-a\right) \equiv 0, \quad f \in C_{0}^{1+\beta-\alpha, \frac{1+\beta-\alpha}{2-\alpha}}\left(\bar{Q}^{N-1}\right) .
$$

This allows us to consider $f$ as a function, which is defined for $t \in(-\infty, \infty)$ and which is identical zero in the domain $t<-a$ with the preservation of the smoothness. Let further

$$
f_{1}^{ \pm}(x, t) \in C_{s, 0}^{\gamma, \gamma / 2}\left(\bar{Q}_{ \pm}^{N}\right), \quad f_{2}^{ \pm}\left(x^{\prime}, t\right) \in C_{0}^{2+\beta-\alpha, 1+\gamma / 2}\left(\bar{Q}^{N-1}\right)
$$

are given functions, which are also extended by identical zero in the domain $t<-a$ with the preservation of the classes and have finite support.

Consider the following model problem for the triple of unknown functions $u^{ \pm}(x, t)$ and $\rho\left(x^{\prime}, t\right)$, which are defined on $\bar{Q}_{ \pm}^{N}$ and on $\bar{Q}^{N-1}$ correspondingly:

$$
\begin{gathered}
\frac{\partial u^{ \pm}}{\partial t}-\left( \pm x_{N}\right)^{\alpha} \Delta u^{ \pm}=f_{1}^{ \pm}, \quad(x, t) \in Q_{ \pm}^{N}, \\
u^{ \pm}+A^{ \pm} \rho=f_{2}^{ \pm}, \quad x_{N}=0,\left(x^{\prime}, t\right) \in Q^{N-1}, \\
\rho_{t}-\varepsilon \Delta_{x^{\prime}} \rho+b^{+} \frac{\partial u^{+}}{\partial x_{N}}-b^{-} \frac{\partial u^{-}}{\partial x_{N}}=f\left(x^{\prime}, t\right), \quad x_{N}=0,\left(x^{\prime}, t\right) \in Q^{N-1}, \\
u^{ \pm}(x,-a)=0, \quad \rho\left(x^{\prime},-a\right)=0,
\end{gathered}
$$


$u^{ \pm} \in C_{s, 0}^{2+\gamma, \frac{2+\gamma}{2}}\left(\bar{Q}_{ \pm}^{N}\right), \quad \rho \in C_{0}^{2+\beta-\alpha, 1+\gamma / 2}\left(\bar{Q}^{N-1}\right), \rho_{t} \in C_{0}^{1+\beta-\alpha, \frac{1+\beta-\alpha}{2-\alpha}}\left(\bar{Q}^{N-1}\right)$.

Here $A^{ \pm}, b^{ \pm}, \varepsilon$ are given positive constants and $\varepsilon \in(0,1)$. Note that the term with $\varepsilon$ in (3.5) does not apply directly to Stefan problem and serves as a regularization of the problem that will be needed in the proof of the solvability of the corresponding linearized Stefan problem in an arbitrary domain. To the author's knowledge, this regularization of the boundary condition in the Stefan problem was first used in paper [1]. Let us remark also that problem (3.3)(3.7) is a parabolic problem for degenerate equations with dynamic boundary conditions. To see this it is enough to express the unknown $\rho$ from relation (3.4) as $\rho=-u^{ \pm} / A^{ \pm}+f_{2}^{ \pm} / A^{ \pm}$and then to substitute it in relation (3.5).

Below we prove the following a priori estimate of a solution of problem $(3.3)-(3.7)$.

Theorem 3.1. Let $u^{ \pm}(x, t) \in C_{s, 0}^{2+\gamma, \frac{2+\gamma}{2}}\left(\bar{Q}_{ \pm}^{N}\right), \rho \in C_{0}^{3+\beta-\alpha, 1+\frac{1+\beta-\alpha}{2-\alpha}}\left(\bar{Q}^{N-1}\right)$ give a solution with finite support of problem (3.3)-(3.7). Then for arbitrary $T>0$ the following estimate is valid

$$
\begin{aligned}
\mathcal{U}(T) \equiv & \left|u^{+}\right|_{s, R_{+T}^{N, a}}^{(2+\gamma)}+\left|u^{-}\right|_{s, R_{-T}^{N, a}}^{(2+\gamma)}+\varepsilon \sum_{i, j=1}^{N-1}\left|\rho_{x_{i} x_{j}}\right|_{C^{1+\beta-\alpha, \frac{1+\beta-\alpha}{2-\alpha}}}\left(R_{T}^{N-1, a}\right) \\
& +|\rho|_{R_{T}^{N-1, a}}^{\left(2+\beta-\alpha, \frac{2+\beta-\alpha}{2-\alpha}\right)}+\left|\rho_{t}\right|_{R_{T}^{N-1, a}}^{\left(1+\beta-\alpha, \frac{1+\beta-\alpha}{2-\alpha}\right)} \\
\leq & C_{T}\left(\left|f_{1}^{+}\right|_{s, R_{+T}^{N, a}}^{(\gamma)}+\left|f_{1}^{-}\right|_{s, R_{-T}^{N, a}}^{(\gamma)}+\left|f_{2}^{+}\right|_{C^{2+\beta-\alpha, \frac{2+\beta-\alpha}{2-\alpha}}}\left(R_{T}^{N-1, a}\right)\right. \\
& \left.+\left|f_{2}^{-}\right|_{C^{2+\beta-\alpha, \frac{2+\beta-\alpha}{2-\alpha}}\left(R_{T}^{N-1, a}\right)}+|f|_{C^{1+\beta-\alpha, \frac{1+\beta-\alpha}{2-\alpha}}\left(R_{T}^{N-1, a}\right)}\right) \equiv C_{T} \mathcal{M}(T),
\end{aligned}
$$

where the constant $C_{T}$ in (3.8) does not depend on $\varepsilon \in(0,1)$.

Subsequent content of this section is devoted to the proof of Theorem 3.1.

Note first that by Lemmas 2.6 and 2.2 we can without loss of generality assume that

$$
f_{1}^{ \pm} \equiv 0, \quad f_{2}^{ \pm} \equiv 0
$$

since the general case can be reduced to the specified one by the change of the unknown functions $u^{ \pm}=v^{ \pm}+w^{ \pm}$, where $v^{ \pm}$are the new unknowns, and $w^{ \pm}$ satisfy (3.3) with the boundary conditions

$$
\left.w^{ \pm}\right|_{x_{N}=0}=f_{2}^{ \pm}\left(x^{\prime}, t\right) .
$$

Thus, further we assume that only the function $f\left(x^{\prime}, t\right)$ is nonzero in the righthand sides of (3.3)-(3.5).

In addition, because the right sides of relations (3.3)-(3.5) belong to the classes with zero at the bottom and because of conditions (3.6), (3.7) we can 
consider that relation (3.3)-(3.5) are valid for $t<-a$ assuming that all the functions are extended by zero to this domain.

An important point of proving (3.8) is to prove the following a priori estimate.

Lemma 3.2. Under the conditions of Theorem 3.1 and under condition (3.9) the following estimate is valid

$$
\begin{aligned}
\left\langle\nabla_{x^{\prime}} \rho\right\rangle_{x^{\prime}, R_{T}^{N-1, a}}^{(1+\beta-\alpha)} & \leq C_{T}\left(\left|\nabla_{x^{\prime}} u^{+}\right|_{R_{+T}^{N, a}}^{(0)}+\left|\nabla_{x^{\prime}} u^{-}\right|_{R_{-T}^{N, a}}^{(0)}+\left|\nabla_{x^{\prime}} \rho\right|_{R_{T}^{N-1, a}}^{(0)}+\mathcal{M}(T)\right) \\
& \equiv C_{T}(\mathcal{N}(T)+\mathcal{M}(T)) \leq C_{T}(T+a)^{\delta} \mathcal{U}(T)+C_{T} \mathcal{M}(T),
\end{aligned}
$$

where

$$
\mathcal{N}(T) \equiv\left|\nabla_{x^{\prime}} u^{+}\right|_{R_{+T}^{N, a}}^{(0)}+\left|\nabla_{x^{\prime}} u^{-}\right|_{R_{-T}^{N, a}}^{(0)}+\left|\nabla_{x^{\prime}} \rho\right|_{R_{T}^{N-1, a}}^{(0)}
$$

To obtain the last inequality in (3.11) we use estimates (2.1)-(2.4).

Proof. Denote for brevity $l=1+\beta-\alpha$ and fix a point $\left(x_{0}^{\prime}, t_{0}\right)$ in the set $R_{T}^{N-1, a}$. We are going to apply the method of paper [53]. So in order to maintain the succession of the notation with this paper we will assume without loss of generality that $\left(x_{0}^{\prime}, t_{0}\right)=(0,0)$. This choice is not important as can be seen from the following proof. Suppose further that $O=\left(x^{\prime}=0, x_{N}=0, t=0\right)$ is the corresponding point in $R_{+T}^{N, a}$. We show that for every $h \in(0,1)$ and for any $i, j=\overline{1, N-1}$ we have the following inequality

$$
\left|\rho_{x_{i}}\left(\vec{e}_{j} h, 0\right)-\rho_{x_{i}}\left(-\vec{e}_{j} h, 0\right)\right| \leq C_{T}(\mathcal{N}(T)+\mathcal{M}(T)) h^{l},
$$

where $\vec{e}_{j}$ is the unit vector of the $O x_{j}$-axis. Since the point $O$ and the step $h \in(0,1)$ in relation $(3.12)$ are arbitrary, estimate $(3.11)$ of the lemma follows from estimate (3.12).

Let $y_{1}, y_{2} \in[0,1], y \equiv\left(y_{1}, y_{2}\right)$ and let also $i, j \in\{1,2, \ldots, N-1\}$ are fixed. Consider the differences

$$
\begin{aligned}
& v^{ \pm}\left(x, t, y_{1}, y_{2}\right)=\Delta_{i, y_{1}} \Delta_{j, y_{2}} u^{ \pm}(x, t) \\
& =u^{ \pm}\left(x+y_{1} \vec{e}_{i}+y_{2} \vec{e}_{j}, t\right)-u^{ \pm}\left(x-y_{1} \vec{e}_{i}+y_{2} \vec{e}_{j}, t\right) \\
& \quad-u^{ \pm}\left(x+y_{1} \vec{e}_{i}-y_{2} \vec{e}_{j}, t\right)+u^{ \pm}\left(x+y_{1} \vec{e}_{i}+y_{2} \vec{e}_{j}, t\right),
\end{aligned}
$$

where

$$
\Delta_{k, h} u(x, t) \equiv u\left(x+h \vec{e}_{k}, t\right)-u\left(x-h \vec{e}_{k}, t\right)
$$

Denote also

$$
r\left(x^{\prime}, t, y_{1}, y_{2}\right)=\Delta_{i, y_{1}} \Delta_{j, y_{2}} \rho\left(x^{\prime}, t\right)
$$

Note that

$$
\frac{\partial^{2} v^{ \pm}}{\partial x_{i}^{2}}-\frac{\partial^{2} v^{ \pm}}{\partial y_{1}^{2}}=0, \quad \frac{\partial^{2} v^{ \pm}}{\partial x_{j}^{2}}-\frac{\partial^{2} v^{ \pm}}{\partial y_{2}^{2}}=0
$$


Therefore in the domains $R_{ \pm}^{N} \times\{-\infty<t<T\} \times\left\{0<y_{1}<1\right\} \times\left\{0<y_{2}<1\right\}$ the functions $v^{ \pm}(x, t, y)$ satisfy the equations

$$
\begin{aligned}
& L^{*} v^{ \pm} \equiv \frac{\partial v^{ \pm}}{\partial t} \\
& \quad-\left( \pm x_{N}\right)^{\alpha}\left(\sum_{k \neq i, j} \frac{\partial^{2} v^{ \pm}}{\partial x_{k}^{2}}+\frac{3}{4} \frac{\partial^{2} v^{ \pm}}{\partial x_{i}^{2}}+\frac{3}{4} \frac{\partial^{2} v^{ \pm}}{\partial x_{j}^{2}}+\frac{1}{4} \frac{\partial^{2} v^{ \pm}}{\partial y_{1}^{2}}+\frac{1}{4} \frac{\partial^{2} v^{ \pm}}{\partial y_{2}^{2}}\right)=0
\end{aligned}
$$

Also note that

$$
\begin{aligned}
\left|v^{ \pm}\right| & =\left|\Delta_{i, y_{1}} \Delta_{j, y_{2}} u^{ \pm}(x, t)\right| \\
& =y_{2}\left|\Delta_{i, y_{1}} \int_{-1}^{1} u_{x_{j}}^{ \pm}\left(x+\omega y_{2} \vec{e}_{j}\right) d \omega\right| \leq 4 y_{2} \mathcal{N}(T) .
\end{aligned}
$$

Exactly the same way

$$
\left|v^{ \pm}\right| \leq 4 y_{1} \mathcal{N}(T)
$$

and therefore

$$
\left|v^{ \pm}\right| \leq 4 y_{\min } \mathcal{N}(T)
$$

where

$$
y_{\min }=\min \left\{y_{1}, y_{2}\right\}
$$

Similarly, we have

$$
|r| \leq 4 y_{\min } \mathcal{N}(T)
$$

Denote

$$
\begin{aligned}
y=\left(y_{1}, y_{2}\right), \quad P^{ \pm}= & \left\{(x, t, y):\left|x_{m}\right|<1, m=\overline{1, N-1}, 0< \pm x_{N}<1,\right. \\
& \left.-1<t<0,0<y_{k}<1, k=1,2\right\} .
\end{aligned}
$$

Denote also

$$
\Sigma^{ \pm}=\partial P^{ \pm} \backslash\left(\{t=0\} \cup\left\{x_{N}=0\right\}\right), \quad \Sigma_{0}=\partial P^{ \pm} \cap\left\{x_{N}=0\right\},
$$

that is, $\Sigma^{ \pm}$are parabolic boundaries of the parallelepipeds $P^{ \pm}$without their common part $\left\{x_{N}=0\right\}$. This common part will be denoted by $\Sigma_{0}$. problem

In the parallelepipeds $P^{ \pm}$the functions $v^{ \pm}$and $r$ satisfy the following

$$
\begin{gathered}
L^{*} v^{ \pm}=0, \quad(x, t, y) \in P^{ \pm}, \\
\left.v^{ \pm}\right|_{\Sigma^{ \pm}}=g^{ \pm}(x, t, y), \\
v^{ \pm}+A^{ \pm} r=0, \quad x_{N}=0, \\
r_{t}-\varepsilon \Delta_{x^{\prime}} r+b^{+} \frac{\partial v^{+}}{\partial x_{N}}-b^{-} \frac{\partial v^{-}}{\partial x_{N}}=F(x, y, t), \quad x_{N}=0,
\end{gathered}
$$


where

$$
F(x, t, y)=\Delta_{i, y_{1}} \Delta_{j, y_{2}} f, \quad g^{ \pm}(x, t, y)=\Delta_{i, y_{1}} \Delta_{j, y_{2}} u^{ \pm}
$$

Note that in view of assumptions (3.1)

$$
|F(x, t, y)| \leq 2\langle f\rangle_{x_{j}}^{(l)}\left(2 y_{2}\right)^{l} .
$$

A similar inequality with replacing $j$ by $i$ and $y_{2}$ by $y_{1}$ gives similar to (3.18)

$$
|F(x, t, y)| \leq C \mathcal{M}(T) y_{\min }^{l} .
$$

Also note that by $(3.18)$

$$
\left|g^{ \pm}(x, t, y)\right| \leq C \mathcal{N}(T) y_{\min }
$$

To estimate $v^{ \pm}$and $r$ we're going to apply to the problem (3.23)-(3.26) the maximum principle in the following form.

Lemma 3.3. Let functions $H^{ \pm}(x, t, y) \in C^{2,1}\left(P^{ \pm}\right) \cap C^{1,0}\left(\bar{P}^{ \pm}\right), S\left(x^{\prime}, t, y\right) \in$ $C^{2,1}\left(\Sigma_{0}\right)$ satisfy the conditions

$$
\begin{gathered}
L^{*} H^{ \pm} \geq 0, \quad(x, t, y) \in P^{ \pm}, \\
\left.H^{ \pm}\right|_{\Sigma^{ \pm}} \geq 0, \\
H^{ \pm}+A^{ \pm} S=0, \quad x_{N}=0, \\
S_{t}-\varepsilon \Delta_{x^{\prime}} S+b^{+} \frac{\partial H^{+}}{\partial x_{N}}-b^{-} \frac{\partial H^{-}}{\partial x_{N}} \leq 0, \quad x_{N}=0 .
\end{gathered}
$$

Then

$$
H^{ \pm} \geq 0, \quad(x, t, y) \in \bar{P}^{ \pm} ; \quad S \leq 0, \quad\left(x^{\prime}, t, y\right) \in \Sigma_{0} .
$$

We do not give a detailed proof of this lemma since it uses standard arguments. We only note that the functions $H^{ \pm}$can not reach a negative minimum at $\left\{x_{N}=0\right\}$. In this case by (3.32) they would reached a negative minimum simultaneously and corresponding point would be, again by (3.32), a point of a positive maximum of the function $S$. All this together in the standard way contradicts boundary condition (3.33).

We shall need the auxiliary functions $w^{ \pm}(x, t)$ defined on

$$
\Pi^{ \pm}=\left\{\left|x_{m}\right| \leq 1, m=\overline{1, N-1}, 0 \leq \pm x_{N} \leq 1,-1 \leq t \leq 0\right\}
$$

correspondingly and such that

$$
\begin{gathered}
L_{x t}^{*} w^{ \pm}=\frac{\partial w^{ \pm}}{\partial t}- \\
-\left( \pm x_{N}\right)^{\alpha}\left(\sum_{k \neq i, j} \frac{\partial^{2} w^{ \pm}}{\partial x_{k}^{2}}+\frac{3}{4} \frac{\partial^{2} w^{ \pm}}{\partial x_{i}^{2}}+\frac{3}{4} \frac{\partial^{2} w^{ \pm}}{\partial x_{j}^{2}}\right)=0, \quad x_{N} \neq 0 \\
\left.w^{ \pm}\right|_{\left\{\left|x_{k}\right|=1\right\} \cup\{t=-1\}} \geq \nu>0 \\
w^{ \pm}(0,0)=0,\left.\quad w^{ \pm}\right|_{\Pi^{ \pm}} \geq 0
\end{gathered}
$$




$$
w^{ \pm}(x, t) \in C^{2,1}\left(\Pi^{ \pm} \cap\left\{x_{N}=0\right\}\right) .
$$

Such functions can be constructed as follows. Consider, for example, $w^{+}(x, t)$. Let $G^{+}(x, t)$ be a function from $C^{\infty}$ in $\overline{R_{+}^{N}} \times(-\infty, \infty)$ such that $G^{+} \equiv 0$ for $|x|+|t| \leq 1 / 4$ and for $t \leq-2,|x| \geq 2$ and $G^{+}>0$ in the other points of $\overline{R_{+}^{N}} \times(-\infty, \infty)$. Let $w^{+}(x, t)$ be the solution of the following initial boundary value problem in half-space

$$
\begin{gathered}
L_{x, t}^{*} w^{+}=0, \quad x_{N}>0, t>-2, \\
\left.w^{+}\right|_{x_{N}=0}=G^{+}(x, t) \in C^{\infty}, \\
\left.w^{+}\right|_{t=-2}=0 .
\end{gathered}
$$

Lemma 2.6 implies that the function $w^{+}$exists in the appropriate class and

$$
\left|w^{+}\right|_{s, \overline{R_{+}^{N}} \times[-2,0]}^{(2+\gamma)} \leq C\left(G^{+}\right) .
$$

Because of the properties of $G^{+}(x, t)$ and by the strong maximum principle (see [59]) the function $w^{+}$has all desirable properties including (3.36).

Now consider the following comparison functions defined on $\bar{P}^{ \pm}$. Denote

$$
\begin{gathered}
\varphi(y)=y_{1} y_{2}\left(y_{1}^{l}+y_{2}^{l}\right)^{-\frac{1}{l}+1}, \\
\psi^{ \pm}\left(x_{N}, y\right)=y_{1} y_{2}\left[\left(y_{1} \pm x_{N}\right)^{l}+\left(y_{2} \pm x_{N}\right)^{l}\right]^{-\frac{1}{l}+1}, \quad \pm x_{N} \geq 0, \\
\theta^{ \pm}(x, t, y)= \begin{cases}\left(y_{1}^{-1}+y_{2}^{-1}\right)^{-1} w^{ \pm}(x, t), & y_{\min }>0, \\
0, & y_{\min }=0 .\end{cases}
\end{gathered}
$$

The direct verification shows (cf. [53]), that the functions $\varphi$ and $\psi^{ \pm}$possess properties

$$
\begin{gathered}
\pm\left.\frac{\partial \psi^{ \pm}}{\partial x_{N}}\right|_{x_{N}=0} \leq-\nu y_{\min }^{l}, \\
\left|L^{*} \psi^{ \pm}\right| \leq C\left|x_{N}\right|^{\alpha} y_{\min }^{-1+l}, \\
\left.\frac{\partial \varphi}{\partial x_{N}}\right|_{x_{N}=0}=0, \\
L^{*} \varphi \geq \nu\left|x_{N}\right|^{\alpha} y_{\min }^{-1+l}, \\
\left.\varphi\right|_{y_{k}=1} \geq \nu y_{\min } .
\end{gathered}
$$

Thus, if we choose a sufficiently large constant $K>0$ the functions

$$
h^{ \pm} \equiv \psi^{ \pm}+K \varphi
$$

will have the properties

$$
\begin{gathered}
\pm\left.\frac{\partial h^{ \pm}}{\partial x_{N}}\right|_{x_{N}=0} \leq-\nu y_{\min }^{l}, \\
L^{*} h^{ \pm} \geq \nu\left|x_{N}\right|^{\alpha} y_{\min }^{-1+l}>0, \quad(x, t, y) \in P^{ \pm},
\end{gathered}
$$




$$
\left.h^{ \pm}\right|_{y_{k}=1} \geq \nu y_{\min }
$$

At the same time the functions $\theta^{ \pm}(x, t, y)$ have the properties

$$
\begin{gathered}
L^{*} \theta^{ \pm} \geq 0, \quad(x, t, y) \in P^{ \pm}, \\
\left|\frac{\partial \theta^{ \pm}}{\partial x_{N}}\right|_{x_{N}=0} \leq C y_{\min }, \\
\left.\theta^{ \pm}\right|_{\cup_{k}\left\{\left|x_{k}\right|=1\right\} \cup\{t=-1\}} \geq \nu y_{\min } .
\end{gathered}
$$

Consider now the following comparison functions

$$
\begin{aligned}
H^{ \pm}(x, t, y) & \equiv A^{ \pm}\left[L_{1} \theta^{ \pm}(x, t, y)+L_{2} h^{ \pm}\left(x_{N}, y\right)\right](N(T)+M(T)), \\
S\left(x^{\prime}, t, y\right) & =-\left[L_{1} \theta^{ \pm}\left(x^{\prime}, 0, t, y\right)+L_{2} h^{ \pm}(0, y)\right](N(T)+M(T)),
\end{aligned}
$$

where $L_{1}$ and $L_{2}$ are some positive constants.

Choosing first $L_{1}$ and then $L_{2}$ sufficiently large and using on one hand (3.28), (3.29), and on the other hand (3.49)-(3.54), we see that the triple of the functions

$$
H^{+} \pm v^{+}(x, t, y), \quad H^{-} \pm v^{-}(x, t, y), \quad S \pm r\left(x^{\prime}, t, y\right)
$$

satisfies in $P^{ \pm}$the conditions of Lemma 3.3. Hence,

$$
H^{+} \pm v^{+} \geq 0, \quad H^{-} \pm v^{-} \geq 0, \quad S \pm r \leq 0,
$$

that is,

$$
\left|v^{ \pm}\right| \leq C H^{ \pm}, \quad|r| \leq C|S|, \quad(x, t, y) \in \bar{P}^{ \pm} .
$$

Take in (3.57) $x=0, t=0$. Then in view of $\theta^{ \pm}(0,0, y)=0$ we obtain for $v^{+}\left(0,0, y_{1}, y_{2}\right)$ similarly [53]

$\left|v^{+}\left(0,0, y_{1}, y_{2}\right)\right|=\left|\Delta_{i, y_{1}} \Delta_{j, y_{2}} u^{+}(0,0)\right| \leq C[\mathcal{N}(T)+\mathcal{M}(T)] y_{1} y_{2}\left(y_{1}^{l}+y_{2}^{l}\right)^{-\frac{1}{l}+1}$.

Dividing both sides of this relation by $y_{1}$ and taking the limit as $y_{1} \rightarrow 0$ we obtain

$$
\left|\frac{\partial u^{+}}{\partial x_{i}}\left(0+y_{2} \vec{e}_{j}, 0\right)-\frac{\partial u^{+}}{\partial x_{i}}\left(0-y_{2} \vec{e}_{j}, 0\right)\right| \leq C[\mathcal{N}(T)+\mathcal{M}(T)] y_{2}^{l},
$$

and similarly

$$
\left|\frac{\partial \rho}{\partial x_{i}}\left(0+y_{2} \vec{e}_{j}, 0\right)-\frac{\partial \rho}{\partial x_{i}}\left(0-y_{2} \vec{e}_{j}, 0\right)\right| \leq C[\mathcal{N}(T)+\mathcal{M}(T)] y_{2}^{l}
$$

Since all of the above arguments are valid for any $\left(x_{0}^{\prime}, t_{0}\right) \in R_{T}^{N-1, a}$, by the same token estimate (3.11) and Lemma 3.2 are proved.

We continue the proof of the theorem. From (3.5) it follows that

$$
\rho_{t}-\varepsilon \Delta_{x^{\prime}} \rho=F\left(x^{\prime}, t\right) \equiv-b^{+} \frac{\partial u^{+}}{\partial x_{N}}+b^{-} \frac{\partial u^{-}}{\partial x_{N}}+f .
$$


Moreover, in view of inequalities (1.33), (2.1), (2.4)

$$
\begin{aligned}
|F|_{R_{T}^{N-1}}^{(\gamma)} & \leq C\left(\left|\nabla u^{+}\right|_{R_{T}^{N-1, a}}^{(\gamma)}+\left|\nabla u^{-}\right|_{R_{T}^{N-1, a}}^{(\gamma)}\right)+C \mathcal{M}(T) \\
& \leq C(T+a)^{\mu} \mathcal{U}(T)+C \mathcal{M}(T),
\end{aligned}
$$

and

$$
F\left(x^{\prime}, 0\right) \equiv 0 .
$$

Making in problem (3.60), (3.6) the change of variables $x^{\prime}=\varepsilon^{1 / 2} y$ we obtain the problem

$$
\rho_{t}-\Delta_{y} \rho=\widetilde{F}(y, t), \quad(y, t) \in R_{T}^{N-1, a}, \quad \rho(y,-a)=0,
$$

and

$$
\begin{gathered}
\langle\widetilde{F}(y, t)\rangle_{t, R_{T}^{N-1, a}}^{(\gamma / 2)}=\left\langle F\left(x^{\prime}, t\right)\right\rangle_{t, R_{T}^{N-1, a}}^{(\gamma / 2)}, \\
\langle\widetilde{F}(y, t)\rangle_{y, R_{T}^{N-1, a}}^{(\gamma)}=\varepsilon^{\gamma / 2}\left\langle F\left(x^{\prime}, t\right)\right\rangle_{x^{\prime}, R_{T}^{N-1, a}}^{(\gamma)} .
\end{gathered}
$$

From the arguments of [43], Chapter IV it follows that

$$
\begin{gathered}
\left\langle\rho_{y_{i} y_{j}}\right\rangle_{y, R_{T}^{N-1, a}}^{(\gamma)} \leq C\langle\widetilde{F}(y, t)\rangle_{y, R_{T}^{N-1, a}}^{(\gamma)}, \\
\left\langle\rho_{t}\right\rangle_{t, R_{T}^{N-1, a}}^{(\gamma / 2)} \leq C\left(\langle\widetilde{F}(y, t)\rangle_{y, R_{T}^{N-1, a}}^{(\gamma)}+\langle\widetilde{F}(y, t)\rangle_{t, R_{T}^{N-1, a}}^{(\gamma / 2)}\right) .
\end{gathered}
$$

Making in (3.64), (3.65) the inverse change of variables in view of (3.63) we obtain

$$
\left\langle\rho_{t}\left(x^{\prime}, t\right)\right\rangle_{t, R_{T}^{N-1, a}}^{(\gamma / 2)}+\varepsilon \sum_{i, j}\left\langle\rho_{x_{i} x_{j}}\right\rangle_{x^{\prime}, R_{T}^{N-1, a}}^{(\gamma)} \leq C\left|F\left(x^{\prime}, t\right)\right|_{R_{T}^{N-1, a}}^{(\gamma)} .
$$

Thus, in view of estimate (3.11) of Lemma 3.2 it is proved that $\left\langle\rho_{t}\left(x^{\prime}, t\right)\right\rangle_{t, R_{T}^{N-1, a}}^{(\gamma / 2)}+\left\langle\nabla_{x^{\prime}} \rho\right\rangle_{x^{\prime}, R_{T}^{N-1, a}}^{(1+\beta-\alpha)}+\varepsilon|\rho|_{R_{T}^{N-1, a}}^{(2+\gamma)} \leq C(T+a)^{\mu} \mathcal{U}(T)+C \mathcal{M}(T)$, or, in view of (1.20) ([45]) and due to the finiteness of $\rho$

$$
|\rho|_{C^{2+\beta-\alpha, \frac{2+\beta-\alpha}{2-\alpha}}\left(R_{T}^{N-1, a}\right)}+\varepsilon|\rho|_{R_{T}^{N-1, a}}^{(2+\gamma)} \leq C(T+a)^{\mu} \mathcal{U}(T)+C \mathcal{M}(T),
$$

where the constant $C$ does not depend on $\varepsilon>0$.

Consider now $u^{ \pm}(x, t)$ as the solution of initial-boundary value problem (3.3), (3.4), (3.6). By Lemma 2.6 and estimate (3.67) we conclude that

$$
\left|u^{+}\right|_{s, R_{+T}^{N, a}}^{(2+\gamma)}+\left|u^{-}\right|_{s, R_{-T}^{N, a}}^{(2+\gamma)} \leq C(T+a)^{\mu} \mathcal{U}(T)+C \mathcal{M}(T)
$$

It follows that in condition (3.5)

$$
\begin{gathered}
\left|\frac{\partial u^{+}}{\partial x_{N}}\right|_{C^{1+\beta-\alpha, \frac{1+\beta-\alpha}{2-\alpha}}\left(R_{T}^{N-1, a}\right)}+\left|\frac{\partial u^{-}}{\partial x_{N}}\right|_{C^{1+\beta-\alpha, \frac{1+\beta-\alpha}{2-\alpha}}\left(R_{T}^{N-1, a}\right)} \\
\leq C(T+a)^{\mu} \mathcal{U}(T)+C \mathcal{M}(T)
\end{gathered}
$$


Thus, the function $\rho\left(x^{\prime}, t\right)$ satisfies the Cauchy problem (3.60), (3.6) with the right hand side $F$ and the last has the property

$$
F\left(x^{\prime},-a\right)=0, \quad|F|_{C^{1+\beta-\alpha, \frac{1+\beta-\alpha}{2-\alpha}}\left(R_{T}^{N-1, a}\right)} \leq C(T+a)^{\mu} \mathcal{U}(T)+C \mathcal{M}(T) .
$$

Making again in (3.60), (3.6) the change of variables $x^{\prime}=\varepsilon^{1 / 2} y$, we arrive at the problem of the form $(3.62)$ with $\widetilde{F}$, where $\widetilde{F}$ is such that

$$
\langle\widetilde{F}\rangle_{t, R_{T}^{N-1, a}}^{\left(\frac{1+\beta-\alpha}{2-\alpha}\right)}=\langle F\rangle_{t, R_{T}^{N-1, a}}^{\left(\frac{1+\beta-\alpha}{2-\alpha}\right)}, \quad\langle\widetilde{F}\rangle_{y, R_{T}^{N-1, a}}^{(1+\beta-\alpha)}=\varepsilon^{\frac{1+\beta-\alpha}{2}}\langle F\rangle_{x^{\prime}, R_{T}^{N-1, a}}^{(1+\beta-\alpha)} .
$$

As above, completely similar to [43], Chapter IV, we have the estimates for solutions of problem (3.62)

$$
\begin{gathered}
\left\langle\rho_{t}\right\rangle_{t, R_{T}^{N-1, a}}^{\left(\frac{1+\beta-\alpha}{2-\alpha}\right)} \leq C\langle\widetilde{F}\rangle_{t, R_{T}^{N-1, a}}^{\left(\frac{1+\beta-\alpha}{2-\alpha}\right)} \leq C(T+a)^{\mu} \mathcal{U}(T)+C \mathcal{M}(T), \\
\sum_{i, j=1}^{N-1}\left\langle\rho_{y_{i} y_{j}}\right\rangle_{y, R_{T}^{N-1, a}}^{(1+\beta-\alpha)} \leq C\langle\widetilde{F}\rangle_{y, R_{T}^{N-1, a}}^{(1+\beta-\alpha)}
\end{gathered}
$$

Note that in obtaining estimate (3.71) condition $F\left(x^{\prime},-a\right)=0$ is important.

Proceeding as before and going back to the variables $x^{\prime}$ we find from (3.72) and (3.70) that

$$
\varepsilon \sum_{i, j=1}^{N-1}\left\langle\rho_{x_{i} x_{j}}\right\rangle_{x^{\prime}, R_{T}^{N-1, a}}^{(1+\beta-\alpha)} \leq C\langle F\rangle_{x^{\prime}, R_{T}^{N-1, a}}^{(1+\beta-\alpha)} \leq C(T+a)^{\mu} \mathcal{U}(T)+C \mathcal{M}(T) .
$$

Now combining estimates (3.73), (3.71), (3.68) and (3.67) we find that

$$
\mathcal{U}(T) \leq C(T+a)^{\mu} \mathcal{U}(T)+C \mathcal{M}(T) .
$$

Taking now in (3.74) $T=T_{0}$ so that the value of $T_{0}+a>0$ is sufficiently small we obtain estimate $(3.8)$ on the interval $\left[-a, T_{0}\right]$. We can consider now problem (3.3)-(3.7) on the interval $\left[-a+\left(a+T_{0}\right) / 2,-a+3\left(a+T_{0}\right) / 2\right]$ and remove the initial data with the help of the known functions. This permits us to move up along the $O t$ - axis exactly as in [43], Chapter IV, and we obtain the assertion of Theorem 3.1 on an arbitrary time interval $[-a, T]$.

Thus, Theorem 3.1 is proved.

As a consequence of Theorem 3.1 we have also an estimate for a solution of the following model problem with the dynamic boundary condition. Let a function $u(x, t)$ with finite support satisfies the conditions

$$
\begin{gathered}
\frac{\partial u}{\partial t}-\left(x_{N}\right)^{\alpha} \Delta u=f_{1}, \quad(x, t) \in Q_{+}^{N}, \\
u_{t}-\varepsilon \Delta_{x^{\prime}} u-b \frac{\partial u}{\partial x_{N}}=f\left(x^{\prime}, t\right), \quad x_{N}=0,\left(x^{\prime}, t\right) \in Q^{N-1}, \\
u(x,-a)=0, u(x, t) \in C_{s, 0}^{2+\gamma, \frac{2+\gamma}{2}}\left(\bar{Q}_{+}^{N}\right), \quad u_{t}\left(x^{\prime}, 0, t\right) \in C_{0}^{1+\beta-\alpha, \frac{1+\beta-\alpha}{2-\alpha}}\left(\bar{Q}^{N-1}\right),
\end{gathered}
$$


where $\varepsilon, b>0$,

$$
f_{1}(x, t) \in C_{s, 0}^{\gamma, \frac{\gamma}{2}}\left(\bar{Q}_{+}^{N}\right), \quad f\left(x^{\prime}, t\right) \in C_{0}^{1+\beta-\alpha, \frac{1+\beta-\alpha}{2-\alpha}}\left(\bar{Q}^{N-1}\right) .
$$

Problem (3.75)-(3.77) is obtained from problem (3.3)-(3.7) by a simplification. We drop in (3.3)-(3.7) the unknown $u^{-}(x, t)$, denote $u(x, t) \equiv u^{+}(x, t)$, and put $f_{2}^{+} \equiv 0$. Then we express $\rho\left(x^{\prime}, t\right)$ from relation $(3.4)$ as $\rho\left(x^{\prime}, t\right)=$ $-\frac{1}{A^{+}} u^{+}=-\frac{1}{A^{+}} u\left(x^{\prime}, 0, t\right)$ and substitute this expression in (3.5). Thus the proof of Theorem 3.1 gives the following assertion.

Theorem 3.4. Let $u(x, t)$ be a solution with finite support of problem (3.75)(3.77). Then for arbitrary $T>0$ the following estimate is valid

$$
\begin{aligned}
& |u|_{s, R_{+T}^{N, a}}^{(2+\gamma)}+\varepsilon \sum_{i, j=1}^{N-1}\left|u\left(x^{\prime}, 0, t\right)_{x_{i} x_{j}}\right|_{C^{1+\beta-\alpha, \frac{1+\beta-\alpha}{2-\alpha}}}{\left(R_{T}^{N-1, a}\right)}^{N-1}+\left|u_{t}\left(x^{\prime}, 0 . t\right)\right|_{R_{T}^{N-1, a}}^{\left(1+\beta-\alpha, \frac{1+\beta-\alpha}{2-\alpha}\right)} \\
& \leq C_{T}\left(\left|f_{1}\right|_{s, R_{+T}^{N, a}}^{(\gamma)}+|f|_{C^{1+\beta-\alpha, \frac{1+\beta-\alpha}{2-\alpha}}\left(R_{T}^{N-1, a}\right)}\right),
\end{aligned}
$$

where the constant $C_{T}$ in (3.79) does not depend on $\varepsilon \in(0,1)$.

\section{Reduction of problem (1.3)-(1.7) to a problem in a fixed domain}

Let $\rho(\omega, t)$ be the unknown function defined in Sect. 1, which gives the parameterisation of unknown (free) boundary $\Gamma_{\rho, T}(\rho(\omega, 0) \equiv 0)$. Let also $\rho(x, t)=E \rho(\omega, t)$ be the extension of this function to the whole domain $\overline{\Omega_{T}}$ by the extension operator $E$ from (2.97). We pass in problem (1.3)-(1.7) from the unknown functions $u^{ \pm}(y, \tau)$ to the unknowns $v^{ \pm}(y, \tau)=\left|u^{ \pm}\right|^{m-1} u^{ \pm}(y, \tau)$. Then relations (1.3)-(1.7) take the form

$$
\begin{gathered}
L_{0}\left(v^{ \pm}\right) v^{ \pm} \equiv \frac{\partial v^{ \pm}}{\partial \tau}-a^{ \pm}\left|v^{ \pm}\right|^{\alpha} \nabla_{y}^{2} v^{ \pm}(y, \tau)=0, \quad(y, \tau) \in \Omega_{\rho, T}^{ \pm} \\
v^{+}(y, \tau)=v^{-}(y, \tau)=0, \quad(y, \tau) \in \Gamma_{\rho, T} \\
a^{+} \sum_{i=1}^{N} \cos \left(\vec{N}, y_{i}\right) v_{y_{i}}^{+}-a^{-} \sum_{i=1}^{N} \cos \left(\vec{N}, y_{i}\right) v_{y_{i}}^{-}=k \cos (\vec{N}, \tau), \quad(y, \tau) \in \Gamma_{\rho, T}, \\
v^{ \pm}(y, \tau)=\left|g^{ \pm}\right|^{m-1} g^{ \pm}(y, \tau) \equiv h^{ \pm}(y, \tau), \quad(y, \tau) \in \Gamma_{T}^{ \pm} \\
v^{ \pm}(y, 0)=\left|u_{0}^{ \pm}\right|^{m-1} u_{0}^{ \pm}(y) \equiv v_{0}^{ \pm}(y), \quad y \in \overrightarrow{\Omega^{ \pm}}
\end{gathered}
$$

We make in problem (4.1)-(4.5) the change of variables $(y, \tau)=e_{\rho}(x, t)$, which is defined in (1.31). Denote for simplicity by the same symbols $v^{ \pm}(x, t)$ the unknown functions after this change of variables, that is,

$$
v^{ \pm}(x, t) \equiv v^{ \pm}(y, \tau) \circ e_{\rho}(x, t) .
$$

In view of the properties of $(y, \tau)=e_{\rho}(x, t)$ problem $(4.1)-(4.5)$ in the variables $(x, t)$ reduces to the following problem in the known fixed domains $\Omega_{T}^{ \pm}$for the 
unknown functions $v^{+}, v^{-}, \rho$ (besides $x$-variables we use the corresponding coordinates $(\omega, \lambda)$, which are introduced in (1.28))

$$
\begin{gathered}
L_{\rho}\left(v^{ \pm}\right) v^{ \pm} \equiv \frac{\partial v^{ \pm}}{\partial t}-h_{\rho}^{ \pm} \rho_{t}-\left|v^{ \pm}\right|{ }^{\alpha} \nabla_{\rho}^{2} v^{ \pm}(x, t)=0, \quad(x, t) \in \Omega_{T}^{ \pm}, \\
v^{+}(x, t)=v^{-}(x, t)=0, \quad(x, t) \in \Gamma_{T}, \\
\left(1+\sum_{i, j=1}^{N-1} m_{i j}(x, \rho) \rho_{\omega_{i}} \rho_{\omega_{j}}\right)\left(a^{+} \frac{\partial v^{+}}{\partial \lambda}-a^{-} \frac{\partial v^{-}}{\partial \lambda}\right)+k \rho_{t}\left(1+\rho_{\lambda}\right)=0, \quad(x, t) \in \Gamma_{T}, \\
v^{ \pm}(x, t)-h^{ \pm}(x, t)=0, \quad(x, t) \in \Gamma_{T}^{ \pm}, \\
v^{ \pm}(x, 0)-v_{0}^{ \pm}(x)=0, x \in \overline{\Omega^{ \pm}}, \quad \rho(\omega, 0) \equiv 0, \\
\rho(x, t)=E \rho(\omega, t) .
\end{gathered}
$$

Here $\nabla_{\rho} \equiv \mathcal{E}_{\rho} \nabla_{x}$ and the matrix $\mathcal{E}_{\rho}$ is the conjugate and inverse to the Jacobi matrix of mapping (1.31) for $t=$ const, $m_{i j}(x, \rho)$ are some given smooth functions of their arguments, and

$$
h_{\rho}^{ \pm}(x, t) \equiv \frac{\partial v^{ \pm}}{\partial \lambda} \frac{1}{1+\rho_{\lambda}} .
$$

Note that the last definition is legitimate since the function $\rho(x, t)$ is not identically zero only if $x \in \mathcal{N}$, where the coordinates $(\omega, \lambda) \equiv\left(\omega_{x}, \lambda_{x}\right)$ of a point $x$ are defined. Recall also that the coordinate $\lambda$ is independent of a choice of local coordinates $\omega$. We use the notation with index $\left(\omega_{x}, \lambda_{x}\right)$ to distinguish these coordinates for a point $x$ from the corresponding coordinates $\left(\omega_{y}, \lambda_{y}\right)$ for a point $y$.

Below we explain the derivation of relations (4.6)-(4.10) but here we note the following. Relation (4.8) contains the expression

$$
S_{\rho} \equiv S_{\rho}\left(\omega, \rho, \rho_{\omega}\right) \equiv\left(1+\sum_{i, j=1}^{N-1} m_{i j}(x, \rho) \rho_{\omega_{i}} \rho_{\omega_{j}}\right),
$$

which is explicitly expressed in the local coordinates $\omega$. But in fact the expression $S_{\rho}$ is a function of points of the surface $\Gamma_{T}$ only and its values at points of $\Gamma_{T}$ do not depend on a choice of local coordinates $\omega$. Indeed, first, for any choice of local coordinates $\omega$ condition (4.8) is equivalent to (4.3) and the last is independent of a choice of local coordinates. And secondly, all the other factors and the terms but $S_{\rho}$ in relation (4.8) are invariant with respect to a choice of $\omega$ and they are functions of a point of the surface $\Gamma_{T}$ only. Hence, the expression $S_{\rho}$ as a function of a point of the surface $\Gamma_{T}$ is invariant on a choice of local coordinates $\omega$ as well. And thus, the map $\rho \rightarrow S_{\rho}\left(\omega, \rho, \rho_{\omega}\right)$ defines a nonlinear operator acting on functions defined on $\Gamma_{T}$. This operator is invariant under choice of local coordinates $\omega$, it acts in the space of functions 
on $\Gamma_{T}$ and has a certain expression $S_{\rho}\left(\omega, \rho, \rho_{\omega}\right)$ for every particular choice of the local coordinates $\omega$.

Further, the expression $\frac{\partial v^{ \pm}}{\partial t}-h_{\rho}^{ \pm} \rho_{t}$ is the recalculated in the variables $(x, t)$ derivative $\frac{\partial v^{ \pm}}{\partial \tau}$ after change of variables (1.31):

$$
\frac{\partial v^{ \pm}}{\partial \tau}=\frac{\partial v^{ \pm}}{\partial t} \frac{\partial t}{\partial \tau}+\sum_{i=1}^{N-1} \frac{\partial v^{ \pm}}{\partial \omega_{x i}} \frac{\partial \omega_{x i}}{\partial \tau}+\frac{\partial v^{ \pm}}{\partial \lambda_{x}} \frac{\partial \lambda_{x}}{\partial \tau} .
$$

Here in fact

$$
\frac{\partial t}{\partial \tau}=1, \quad \frac{\partial \omega_{x i}}{\partial \tau}=0
$$

And for the value of $\frac{\partial \lambda_{x}}{\partial \tau}$ due to the relation

$$
\lambda_{x}=\lambda_{y}-\rho(x, t) \circ e_{\rho}^{-1},
$$

and taking into account (4.13) we have

$$
\begin{aligned}
\frac{\partial \lambda_{x}}{\partial \tau} & =-\frac{\partial}{\partial \tau}\left[\rho(x, t) \circ e_{\rho}(x, t)^{-1}\right] \\
& -\frac{\partial \rho}{\partial t} \frac{\partial t}{\partial \tau}-\frac{\partial \rho}{\partial \lambda_{x}} \frac{\partial \lambda_{x}}{\partial \tau}-\sum_{i=1}^{N-1} \frac{\partial \rho}{\partial \omega_{x i}} \frac{\partial \omega_{x i}}{\partial \tau}=-\rho_{t}-\rho_{\lambda_{x}} \frac{\partial \lambda_{x}}{\partial \tau} .
\end{aligned}
$$

So in the variables $x$ and $t$

$$
\frac{\partial \lambda_{x}}{\partial \tau}=-\rho_{t} /\left(1+\rho_{\lambda_{x}}\right) .
$$

Thus, from (4.13) and (4.14) it follows that

$$
\frac{\partial v^{ \pm}}{\partial \tau} \circ e_{\rho}=\frac{\partial v^{ \pm}}{\partial t}-\left[\frac{\partial v^{ \pm}}{\partial \lambda} /\left(1+\rho_{\lambda}\right)\right] \rho_{t}=\frac{\partial v^{ \pm}}{\partial t}-h_{\rho}^{ \pm} \rho_{t} .
$$

We explain further the transition from condition (4.3) to condition (4.8) under change of variables (1.31), as we shall need in the future the exact explicit form of this condition. Define in the neighborhood $\mathcal{N}_{T}$ of the surface $\Gamma_{T}$ the function

$$
\Phi_{\rho}(y, \tau)=\lambda_{x} \circ e_{\rho}^{-1}(y, \tau)=\lambda_{y}-\rho(x, t) \circ e_{\rho}^{-1}(y, \tau)=\lambda(y)-\rho(y, \tau),
$$

where for simplicity we have retained for the function $\rho(x, t) \circ e_{\rho}^{-1}(y, \tau)$ the same notation $\rho(y, \tau)$. By the definition we have $\pm \Phi_{\rho}(y, \tau)>0$ for $(y, \tau) \in \Omega_{\rho, T}^{ \pm}$ and $\Phi_{\rho}(y, \tau)=0$ for $(y, \tau) \in \Gamma_{\rho, T}$. Hence, in (4.3)

$$
\cos \left(\vec{N}, y_{i}\right)=\frac{\Phi_{\rho y_{i}}}{\left|\nabla_{(y, \tau)} \Phi_{\rho}\right|}, \quad \cos (\vec{N}, \tau)=\frac{\Phi_{\rho \tau}}{\left|\nabla_{(y, \tau)} \Phi_{\rho}\right|}
$$

Therefore, relation (4.3) can be written as follows

$$
a^{+}\left(\nabla_{y} v^{+}, \nabla_{y} \Phi_{\rho}\right)-a^{-}\left(\nabla_{y} v^{-}, \nabla_{y} \Phi_{\rho}\right)=k \Phi_{\rho \tau} .
$$

Under change of variables (1.31) the right hand side of (4.16) due to the definition of $\Phi_{\rho}$ takes the form

$$
k \Phi_{\rho \tau}=k \frac{\partial \lambda_{x}}{\partial \tau}=-k \rho_{t} /\left(1+\rho_{\lambda_{x}}\right),
$$


owing to (4.14).

On the other hand, under change of variables (1.31)

$$
\left(\nabla_{y} v^{ \pm}, \nabla_{y} \Phi_{\rho}\right) \circ e_{\rho}(x, t)=\left(\nabla_{\rho} v^{ \pm}, \nabla_{\rho} \lambda_{x}\right) .
$$

Denote by $\Lambda(x)$ the transition matrix from the gradient with respect to the variables $x$ to the gradient with respect to the variables $\left(\omega_{x}, \lambda_{x}\right)$, that is,

$$
\nabla_{x}=\Lambda(x) \nabla_{\left(\lambda_{x}, \omega_{x}\right)} \quad\left(\nabla_{y}=\Lambda(y) \nabla_{\left(\lambda_{y}, \omega_{y}\right)}\right),
$$

where

$$
\Lambda(x)=\left(\begin{array}{cccc}
\frac{\partial \lambda}{\partial x_{1}} & \frac{\partial \omega_{1}}{\partial x_{1}} & \ldots & \frac{\partial \omega_{N-1}}{\partial x_{1}} \\
\ldots & \ldots & \ldots & \ldots \\
\frac{\partial \lambda}{\partial x_{N}} & \frac{\partial \omega_{1}}{\partial x_{N}} & \ldots & \frac{\partial \omega_{N-1}}{\partial x_{N}}
\end{array}\right),
$$

and similarly for the variables $y$. Then in the variables $(x, t)$

$$
\left(\nabla_{\rho} v^{ \pm}, \nabla_{\rho} \lambda_{x}\right)=\left(\mathcal{E}_{\rho} \Lambda \nabla_{(\lambda, \omega)} v^{ \pm}, \mathcal{E}_{\rho} \Lambda \nabla_{(\lambda, \omega)} \lambda_{x}\right) .
$$

Note that $\nabla_{\left(\lambda_{x}, \omega_{x}\right)} \lambda_{x}=\{1,0, \ldots, 0\}$, and also $v^{ \pm} \equiv 0$ on $\Gamma$, hence $\partial v^{ \pm} / \partial \omega_{i}=$ 0 , and therefore

$$
\nabla_{\left(\lambda_{x}, \omega_{x}\right)} v^{ \pm}=\left\{\frac{\partial v^{ \pm}}{\partial \lambda_{x}}, 0, \ldots, 0\right\}=\frac{\partial v^{ \pm}}{\partial \lambda_{x}}\{1,0, \ldots, 0\}=\frac{\partial v^{ \pm}}{\partial \lambda_{x}} \nabla_{\left(\lambda_{x}, \omega_{x}\right)} \lambda_{x}
$$

Thus we obtain

$$
\left(\nabla_{y} v^{ \pm}, \nabla_{y} \Phi_{\rho}\right) \circ e_{\rho}(x, t)=\left(\nabla_{\rho} v^{ \pm}, \nabla_{\rho} \lambda_{x}\right)=\frac{\partial v^{ \pm}}{\partial \lambda_{x}}\left(\nabla_{\rho} \lambda_{x}, \nabla_{\rho} \lambda_{x}\right) .
$$

On the other hand, due to the definition of $\Phi_{\rho}(y, \tau)$

$$
\left(\nabla_{\rho} \lambda_{x}, \nabla_{\rho} \lambda_{x}\right)=\left(\nabla_{y}\left(\lambda_{x} \circ e_{\rho}^{-1}\right), \nabla_{y}\left(\lambda_{x} \circ e_{\rho}^{-1}\right)\right) \circ e_{\rho}=\left(\nabla_{y} \Phi_{\rho}, \nabla_{y} \Phi_{\rho}\right) \circ e_{\rho} .
$$

Using introduced in (4.19) matrix $\Lambda(y)$, we have

$$
\begin{aligned}
\left(\nabla_{y} \Phi_{\rho}, \nabla_{y} \Phi_{\rho}\right) & =\left(\Lambda(y) \nabla_{\left(\lambda_{y}, \omega_{y}\right)} \Phi_{\rho}, \Lambda(y) \nabla_{\left(\lambda_{y}, \omega_{y}\right)} \Phi_{\rho}\right) \\
& =\left(\nabla_{\left(\lambda_{y}, \omega_{y}\right)} \Phi_{\rho}, \Lambda(y)^{*} \Lambda(y) \nabla_{\left(\lambda_{y}, \omega_{y}\right)} \Phi_{\rho}\right) .
\end{aligned}
$$

First, by the definition of $\Phi_{\rho}$

$$
\begin{gathered}
\frac{\partial \Phi_{\rho}}{\partial \lambda_{y}}=\frac{\partial}{\partial \lambda_{y}}\left(\lambda_{y}-\rho(y, \tau)\right)=1-\rho_{\lambda_{y}}, \\
\frac{\partial \Phi_{\rho}}{\partial \omega_{y i}}=\frac{\partial}{\partial \omega_{y i}}\left(\lambda_{y}-\rho(y, \tau)\right)=-\rho_{\omega_{y i}} .
\end{gathered}
$$

In addition, since the coordinate $\lambda_{y}$ is counted along the normal to $\Gamma$, and $\omega_{y i}$ are coordinates on the surface $\Gamma$, we have

$$
\left(\nabla_{y} \lambda(y), \nabla_{y} \lambda(y)\right)=1, \quad\left(\nabla_{y} \lambda(y), \nabla_{y} \omega_{i}(y)\right)=0, i=1, \ldots, N-1 .
$$

Therefore the matrix $\Lambda^{*}(y) \Lambda(y)$ has the form

$$
\Lambda^{*}(y) \Lambda(y)=\left(\begin{array}{ccccc}
1 & 0 & 0 & \ldots & 0 \\
0 & m_{11} & m_{12} & \ldots & m_{1(N-1)} \\
\ldots & \ldots & \ldots & \ldots & \ldots \\
0 & m_{(N-1) 1} & m_{(N-1) 2} & \ldots & m_{(N-1)(N-1)}
\end{array}\right)
$$


where

$$
m_{i j}=m_{j i}=\left(\nabla_{y} \omega_{i}(y), \nabla_{y} \omega_{j}(y)\right)-
$$

are some smooth functions. Thus,

$$
\left(\nabla_{\left(\lambda_{y}, \omega_{y}\right)} \Phi_{\rho}, \Lambda^{*}(y) \Lambda(y) \nabla_{\left(\lambda_{y}, \omega_{y}\right)} \Phi_{\rho}\right)=\left(1-\rho_{\lambda_{y}}\right)^{2}+\sum_{i, j=1}^{N-1} m_{i j}(y) \rho_{\omega_{y i}} \rho_{\omega_{y j}} .
$$

Make now in (4.27) change of variables (1.31), and recalculate the derivatives of $\rho$ with respect to $\left(\lambda_{y}, \omega_{y}\right)$ in terms of the derivatives with respect to $\left(\lambda_{x}, \omega_{x}\right)$. We have

$$
\rho_{\lambda_{y}} \circ e_{\rho}=\rho_{t} \frac{\partial t}{\partial \lambda_{y}}+\rho_{\lambda_{x}} \frac{\partial \lambda_{x}}{\partial \lambda_{y}}+\sum_{i=1}^{N-1} \rho_{\omega_{x i}} \frac{\partial \omega_{x i}}{\partial \lambda_{y}} .
$$

From the definition of the mapping $e_{\rho}$ it follows that

$$
\frac{\partial t}{\partial \lambda_{y}}=0, \quad \frac{\partial \omega_{x i}}{\partial \lambda_{y}}=0
$$

At the same time by (4.28), (4.29)

$$
\frac{\partial \lambda_{x}}{\partial \lambda_{y}}=1-\rho_{\lambda_{y}}=1-\rho_{\lambda_{x}} \frac{\partial \lambda_{x}}{\partial \lambda_{y}},
$$

that is,

$$
\frac{\partial \lambda_{x}}{\partial \lambda_{y}}=\frac{1}{1+\rho_{\lambda_{x}}}
$$

Therefore, by (4.28), (4.29) and (4.30)

$$
\rho_{\lambda_{y}} \circ e_{\rho}=\frac{\rho_{\lambda_{x}}}{1+\rho_{\lambda_{x}}} .
$$

Further,

$$
\rho_{\omega_{y i}} \circ e_{\rho}=\rho_{t} \frac{\partial t}{\partial \omega_{y i}}+\rho_{\lambda_{x}} \frac{\partial \lambda_{x}}{\partial \omega_{y i}}+\sum_{j=1}^{N-1} \rho_{\omega_{x i}} \frac{\partial \omega_{x j}}{\partial \omega_{y i}}
$$

and

$$
\frac{\partial t}{\partial \omega_{y i}}=0, \quad \frac{\partial \omega_{x j}}{\partial \omega_{y i}}=\delta_{i j}, \quad i, j=1, \ldots, N-1 .
$$

At the same time

$$
\frac{\partial\left(\lambda_{x} \circ e_{\rho}\right)}{\partial \omega_{y i}}=\left[\frac{\partial}{\partial \omega_{y i}}\left(\lambda_{y}-\rho(y, \tau)\right)\right] \circ e_{\rho}=-\rho_{\omega_{y i}} \circ e_{\rho},
$$

That is, by virtue of (4.32) and (4.33),

$$
\rho_{\omega_{y i}} \circ e_{\rho}=\rho_{\lambda_{x}}\left(-\rho_{\omega_{y i}} \circ e_{\rho}\right)+\rho_{\omega_{x i}},
$$

hence by (4.34),

$$
\rho_{\omega_{y i}} \circ e_{\rho}=\frac{\rho_{\omega_{x i}}}{1+\rho_{\lambda_{x}}} .
$$


Thus, from (4.21), (4.27), (4.31) and (4.35) it follows that in (4.21)

$$
\left(\nabla_{\rho} \lambda_{x}, \nabla_{\rho} \lambda_{x}\right)=\frac{1}{\left(1+\rho_{\lambda_{x}}\right)^{2}}\left[1+\sum_{i, j=1}^{N-1} m_{i j}(x, \rho) \rho_{\omega_{x i}} \rho_{\omega_{x j}}\right] .
$$

Finally, relation (4.8) follows from relations (4.16), (4.17), (4.21) and (4.36).

\section{Properties of the nonlinear operator of problem (4.6)-(4.11) and it's Frechet derivative}

Our goal in this section is to calculate the Frechet derivative of the nonlinear operator of problem (4.6)-(4.11). This operator is defined by the left-hand sides of relations (4.6)-(4.11). The domain of definition of this operator will be a small neighbourhood of some approximate solution to problem (4.6)(4.11). So we first construct such an approximate solution. This will be done by extending the initial data $\left(v_{0}^{+}, v_{0}^{-}, 0\right)$ from $t=0$ to the region $t \in[0, T]$ up to some functions $\left(w^{+}, w^{-}, \sigma\right)$ in a way to ensure conditions (4.6)-(4.11) at $t=0$. Then for sufficiently small $T>0$ the triple $\left(w^{+}, w^{-}, \sigma\right)$ will give an approximate solution to (4.6)-(4.11).

Note that from equations (4.6), (4.8) and from initial data (4.10) we can calculate the derivatives with respect to time $\partial v^{ \pm} / \partial t$ and $\partial \rho / \partial t$ at $t=0$ :

$$
\begin{gathered}
\frac{\partial \rho}{\partial t}(\omega, 0)=\left.\rho_{1}(\omega) \equiv \frac{1}{k}\left(a^{+} \frac{\partial v_{0}^{+}}{\partial \lambda}-a^{-} \frac{\partial v_{0}^{-}}{\partial \lambda}\right)\right|_{\Gamma}, \\
\frac{\partial v^{ \pm}}{\partial t}(x, 0)=v_{1}^{ \pm}(x) \equiv \frac{\partial v_{0}^{ \pm}}{\partial \lambda} \rho_{1}+a^{ \pm}\left|v_{0}^{ \pm}(x)\right|^{\alpha} \nabla^{2} v_{0}^{ \pm}(x),
\end{gathered}
$$

and in view of assumptions (1.10), (1.26)

$$
\rho_{1}(\omega) \in C^{1+\beta^{\prime}-\alpha}(\Gamma), \beta^{\prime} \equiv \gamma^{\prime}(1-\alpha / 2)>\beta, \quad v_{1}^{ \pm}(x) \in C_{s}^{\gamma^{\prime}}\left(\overline{\Omega^{ \pm}}\right) .
$$

Note that in view of conditions (1.36) and (4.7) these definitions are agreed at $\Gamma$.

Completely analogous to [43], Chapter IV, on the base of the results of [42] on the solvability of the initial-boundary value problem for degenerate equations (or on the base of Theorem 2.7) we construct functions $w^{ \pm}(x, t) \in$ $C_{s}^{2+\gamma^{\prime}, 1+\gamma^{\prime} / 2}\left(\overline{\Omega_{T}^{ \pm}}\right)$such that

$$
\left.\left|w^{ \pm}\right|_{s, \overline{\Omega_{T}^{ \pm}}}^{\left(2+\gamma^{\prime}\right.}, 1+\gamma^{\prime} / 2\right) \leq C\left(\left|v_{0}^{ \pm}\right|_{s, \overline{\Omega^{ \pm}}}^{\left(2+\gamma^{\prime}\right)}+\left|v_{1}^{ \pm}\right|_{s, \overline{\Omega^{ \pm}}}^{\left(\gamma^{\prime}\right)} \leq C\left|v_{0}^{ \pm}\right|_{s, \overline{\Omega^{ \pm}}}^{\left(2+\gamma^{\prime}\right.}\right)
$$

and

$$
\left.w^{ \pm}(x, 0)=v_{0}^{ \pm}(x), \frac{\partial w^{ \pm}}{\partial t}(x, 0)=v_{1}^{ \pm}(x),\left.w^{ \pm}(x, t)\right|_{\Gamma_{T}}=0,\left.w^{ \pm}(x, t)\right|_{\Gamma_{T}^{ \pm}}=h \neq 5.5\right)
$$

In addition, just as described in [43], Chapter 4, there is a function $\sigma(\omega, t) \in$ $C^{3+\beta^{\prime}-\alpha, 1+\frac{1+\beta^{\prime}-\alpha}{2}}\left(\Gamma_{T}\right)$ such that

$$
|\sigma|_{\Gamma_{T}}^{\left(3+\beta^{\prime}-\alpha, \frac{3+\beta^{\prime}-\alpha}{2}\right)} \leq C\left(\left|v_{0}^{+}\right|_{s, \overline{\Omega^{+}}}^{\left(2+\gamma^{\prime}\right)}+\left|v_{0}^{-}\right|_{s, \overline{\Omega^{-}}}^{\left(2+\gamma^{\prime}\right)}\right)
$$


and

$$
\sigma(\omega, 0)=\rho(\omega, 0)=0, \quad \frac{\partial \sigma}{\partial t}(\omega, 0)=\rho_{1}(\omega) .
$$

Moreover, by the method described in [43], Chapter IV, the function $\sigma(\omega, t)$ can be extended with the class and with inequality (5.6) to a function defined in $\overline{\Omega_{T}}$ with support in the neighborhood $\mathcal{N} \times[0, T]$ of the surface $\Gamma_{T}$.

We denote the space

$$
P^{2+\beta-\alpha}\left(\Gamma_{T}\right)=\left\{\rho: \rho \in C^{2+\beta-\alpha, \frac{2+\beta-\alpha}{2-\alpha}}\left(\Gamma_{T}\right), \rho_{t} \in C^{1+\beta-\alpha, \frac{1+\beta-\alpha}{2-\alpha}}\left(\Gamma_{T}\right)\right\}
$$

with the norm

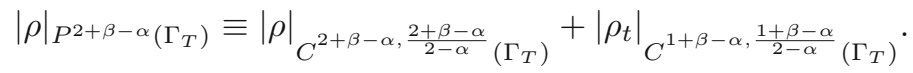

Denote also

$$
\begin{gathered}
\psi=\left(v^{+}, v^{-}, \rho\right) \in \mathcal{H} \equiv C_{s}^{2+\gamma, 1+\gamma / 2}\left(\overline{\Omega_{T}^{+}}\right) \times C_{s}^{2+\gamma, 1+\gamma / 2}\left(\overline{\Omega_{T}^{-}}\right) \times P^{2+\beta-\alpha}\left(\Gamma_{T}\right), \\
\psi_{0}=\left(w^{+}, w^{-}, \sigma\right),
\end{gathered}
$$

and represent relations (4.6)-(4.11) as

$$
\widetilde{F}(\psi)\left(\widetilde{F}^{+}(\psi), \widetilde{F}^{-}(\psi), \widetilde{F}_{1}^{+}(\psi), \widetilde{F}_{1}^{-}(\psi), \widetilde{F}^{0}(\psi), \widetilde{F}_{2}^{+}(\psi), \widetilde{F}_{2}^{-}(\psi), \widetilde{F}_{3}^{+}(\psi), \widetilde{F}_{3}^{+}(\psi)\right)=0
$$

with the non-linear operator of $\psi$, which is defined by the left-hand sides of problem (4.6)-(4.11). Here $\widetilde{F}^{+}(\psi), \widetilde{F}^{-}(\psi)$ are defined by $(4.6), \widetilde{F}_{1}^{+}(\psi), \widetilde{F}_{1}^{-}(\psi)$ are defined by $(4.7), \widetilde{F}^{0}(\psi)$ is defined by $(4.8), \widetilde{F}_{2}^{+}(\psi), \widetilde{F}_{2}^{-}(\psi)$ are defined by (4.9), and $\widetilde{F}_{3}^{+}(\psi), \widetilde{F}_{3}^{+}(\psi)$ are defined by (4.10). Denote further the following space with the elements $\varphi=\left(\theta^{+}, \theta^{-}, \delta\right)$

$$
\begin{gathered}
\mathcal{H}_{0} \equiv\left\{\varphi \in C_{0, s}^{2+\gamma, 1+\gamma / 2}\left(\overline{\Omega_{T}^{+}}\right) \times C_{0, s}^{2+\gamma, 1+\gamma / 2}\left(\overline{\Omega_{T}^{-}}\right) \times P_{0}^{2+\beta-\alpha}\left(\Gamma_{T}\right):\right. \\
\left.\left.\theta^{ \pm}\right|_{\Gamma_{T}} \equiv 0,\left.\quad \theta^{ \pm}\right|_{\Gamma_{T}^{ \pm}} \equiv 0\right\} .
\end{gathered}
$$

Note that if $\varphi \in \mathcal{H}_{0}$ then the triple $\psi_{0}+\varphi$ satisfies initial conditions (4.10) and boundary conditions $(4.7),(4.9)$. This permits instead of the operator $\widetilde{F}$ in (5.11) to consider the operator

$$
F(\varphi)=\left(F^{+}(\varphi), F^{-}(\varphi), F^{0}(\varphi)\right)=\left(\widetilde{F}^{+}\left(\varphi+\psi_{0}\right), \widetilde{F}^{+}\left(\varphi+\psi_{0}\right), \widetilde{F}^{0}\left(\varphi+\psi_{0}\right)\right) .
$$

This operator is defined on $\mathcal{H}_{0}$ by the left-hand sides of relations (4.6), (4.8) with the values in the space

$$
Y=C_{0, s}^{\gamma, \gamma / 2}\left(\overline{\Omega_{T}^{+}}\right) \times C_{0, s}^{\gamma, \gamma / 2}\left(\overline{\Omega_{T}^{-}}\right) \times C_{0}^{1+\beta-\alpha, \frac{1+\beta-\alpha}{2-\alpha}}\left(\Gamma_{T}\right) .
$$

Let us remark that $F$ maps $\mathcal{H}_{0}$ to spaces "with zeros" by the construction of the functions $\psi_{0}=\left(w^{+}, w^{-}, \sigma\right)$. These functions were constructed exactly in the way to ensure that the conditions (4.6), (4.8) are valid at $t=0$. Let

$$
\mathcal{B}_{r}=\mathcal{B}_{r}(0)=\left\{\varphi \in \mathcal{H}_{0}:\|\varphi\| \leq r\right\} \subset \mathcal{H}_{0}, \quad r<\gamma_{0} / 2,
$$


be a ball in $\mathcal{H}_{0}$ of radius $r<\gamma_{0} / 2$ with center at zero. We claim that if the radius $r$ is sufficiently small, then the mapping $F(\varphi)$ is continuously differentiable on $\mathcal{B}_{r}$. Consider first the mapping $F^{0}(\varphi)$, which corresponds to (4.8). The smoothness of the functions $m_{i j}(x, \rho)$ with respect to their arguments is defined solely by the smoothness of the surface $\Gamma$. As this surface is sufficiently smooth, the functions $m_{i j}(x, \rho)$ are continuously differentiable as operators in $\rho$ and consequently they are continuously differentiable as operators in $\varphi$ from $\mathcal{H}_{0}$ to $C_{0}^{1+\beta-\alpha, \frac{1+\beta-\alpha}{2-\alpha}}\left(\Gamma_{T}\right)$. Other terms in the left-hand side of (4.8) are evidently continuously differentiable as operators in $\varphi$ from $\mathcal{H}_{0}$ to $C_{0}^{1+\beta-\alpha, \frac{1+\beta-\alpha}{2-\alpha}}\left(\Gamma_{T}\right)$.

Consider now, for example, the operator $F^{+}(\varphi)=\widetilde{F}^{+}\left(\varphi+\psi_{0}\right)$, which is defined by the left-hand side of (4.6). Write it in the form (recall that $\left.\nabla_{\rho}=E_{\rho} \nabla, E_{\rho}=\left(\left.e_{i j}(x, \rho, \nabla \rho)\right|_{i, j=\overline{1, N}}\right)\right)$

$$
\begin{aligned}
F^{+}(\varphi) & =\frac{\partial v^{+}}{\partial t}-\left(\frac{\partial v^{+}}{\partial \lambda} \frac{1}{1+\rho_{\lambda}}\right) \frac{\partial \rho}{\partial t}-\left(v^{+}\right)^{\alpha} \nabla_{\rho}^{2} v^{+} \\
& =\frac{\partial v^{+}}{\partial t}-\left(\frac{\partial v^{+}}{\partial \lambda} \frac{1}{1+\rho_{\lambda}}\right) \frac{\partial \rho}{\partial t} \\
& -\left(\frac{v^{+}}{\lambda(x)}\right)^{\alpha} \sum_{i, j=1}^{N}\left(\sum_{k, l=1}^{N} e_{l, i}(x, \rho, \nabla \rho) e_{k j}(x, \rho, \nabla \rho)\right)\left(\lambda(x) \frac{\partial^{2} v^{+}}{\partial x_{i} \partial x_{j}}\right) \\
& -\left(\frac{v^{+}}{\lambda(x)}\right)^{\alpha} \sum_{i, l=1}^{N}\left(\sum_{k, j=1}^{N} e_{l i} \frac{\partial e_{k j}(x, \rho, \nabla \rho)}{\partial \rho_{x_{l}}} \frac{\partial v^{+}}{\partial x_{j}}\right)\left(\lambda(x) \frac{\partial^{2} \rho}{\partial x_{i} \partial x_{l}}\right) \\
& -\left(\frac{v^{+}}{\lambda(x)}\right)^{\alpha} \lambda(x)^{\alpha} \sum_{i, l, k, j=1}^{N}\left(e_{l i} \frac{\partial e_{k j}(x, \rho, \nabla \rho)}{\partial \rho} \frac{\partial \rho}{\partial x_{i}}+e_{l i} \frac{\partial e_{k j}(x, \rho, \nabla \rho)}{\partial x_{i}}\right) \frac{\partial v^{+}}{\partial x_{j}} .
\end{aligned}
$$

Here $\lambda(x)$ - is extension of the $\lambda$ - coordinate with the preservation of the class from the neighborhood $\mathcal{N}$ of the surface $\Gamma$ on all $\bar{\Omega}$ to a function satisfying the conditions

$$
\nu d^{+}(x) \leq \lambda(x) \leq \nu^{-1} d^{+}(x)
$$

retaining for her the same notation. Again, the functions $e_{i j}(x, \rho, \nabla \rho)$, $\frac{\partial e_{k j}(x, \rho, \nabla \rho)}{\partial \rho_{x_{l}}}, \frac{\partial e_{k j}(x, \rho, \nabla \rho)}{\partial \rho}$, and $\frac{\partial e_{k j}(x, \rho, \nabla \rho)}{\partial x_{i}}$ are sufficiently smooth with respect to their arguments because of the smoothness of the surface $\Gamma$ in view of the definition of the mapping $e_{\rho}(x, t)$ in $(1.30),(1.31)$. Moreover, the expression for $F^{+}(\varphi)$ in (5.16) is linear with respect to weighted second derivatives $\lambda(x) \frac{\partial^{2} v^{+}}{\partial x_{i} \partial x_{j}}$ and $\lambda(x) \frac{\partial^{2} \rho}{\partial x_{i} \partial x_{l}}$. So $F^{+}(\varphi)$ is smooth with respect to these arguments as a mapping from $\mathcal{H}_{0}$ to $C_{0, s}^{\gamma, \gamma / 2}\left(\overline{\Omega_{T}^{+}}\right)$. Thus it is enough to consider the smoothness of the mapping

$$
\varphi \rightarrow\left(\frac{v^{+}}{\lambda(x)}\right)^{\alpha}=\left(\frac{w^{+}(x, t)+\theta^{+}(x, t)}{\lambda(x)}\right)^{\alpha}
$$


Since $v_{0}, v^{+}=0$ for $x \in \Gamma$, then for $x \in \mathcal{N}$

$$
\frac{v^{+}(x, t)}{\lambda(x)}=\int_{0}^{1} \frac{\partial v^{+}}{\partial \lambda}(\lambda s, \omega, t) d s, \quad \frac{v_{0}^{+}}{\lambda}=\int_{0}^{1} \frac{\partial v_{0}^{+}}{\partial \lambda}(\lambda s, \omega) d s,
$$

where we assume that the neighborhood $\mathcal{N}$ is so small that

$$
\frac{\partial v_{0}^{+}}{\partial \lambda} \geq \nu>0, \quad x \in \mathcal{N} .
$$

In addition, we assume that $T \leq T_{0}$ is so small that

$$
\frac{\partial w^{+}}{\partial \lambda}(x, t) \geq \nu>0, \quad(x, t) \in \mathcal{N} \times[0, T], \quad T \leq T_{0} .
$$

Assuming now that the radius $r=r(\nu)$ of the ball $\mathcal{B}_{r} \ni \varphi$ is sufficiently small $r \leq r_{0}(\nu)$, we can assume that for $\varphi=\left(\theta^{+}, \theta^{-}, \delta\right) \in \mathcal{B}_{r}$

$$
\frac{\partial v^{+}}{\partial \lambda}(x, t)=\frac{\partial w^{+}}{\partial \lambda}(x, t)+\frac{\partial \theta^{+}}{\partial \lambda}(x, t) \geq \nu>0, \quad(x, t) \in N \times[0, T], r \leq r_{0}(\nu), \quad T \leq T_{0} .
$$

At the same time outside the neighborhood $\mathcal{N}_{T}$ holds

$$
v_{0}^{+}(x) \geq \nu>0, \quad x \in \overline{\Omega^{+}} \backslash \mathcal{N} .
$$

Therefore, assuming as above $T$ and $r$ sufficiently small, we can assume that

$$
v^{+}(x, t)=w^{+}(x, t)+\theta^{+}(x, t) \geq \nu>0, \quad x \in \overline{\Omega^{+}} \backslash N, \quad r \leq r_{0}(\nu), T \leq T_{0} .
$$

Thus we have the representation

$$
\frac{v^{+}(x, t)}{\lambda(x)} \equiv A^{+}(x, t, \varphi)= \begin{cases}\int_{0}^{1} \frac{\partial v^{+}}{\partial \lambda}(\lambda s, \omega, t) d s \geq \nu, & x \in \mathcal{N}, \\ \frac{v^{+}}{\lambda}, & x \in \overline{\Omega^{+}} \backslash \mathcal{N},\end{cases}
$$

and

$$
\left|A^{+}(x, t, \varphi)\right|_{s, \overline{\Omega_{T}^{+}}}^{(\gamma)} \leq C\left(\left|\frac{\partial v^{+}}{\partial \lambda}\right|_{s, \overline{\Omega_{T}^{+}}}^{(\gamma)}+\left|v^{+}\right|_{s, \overline{\Omega_{T}^{+}}}^{(\gamma)}\right) \leq C\left(\|\varphi\|_{\mathcal{H}}\right),
$$

and also

$$
A^{+}(x, t, \varphi) \geq \nu>0 .
$$

From (5.22)-(5.24) it follows that the mapping

$$
\varphi \rightarrow\left(\frac{v^{+}(x, t)}{\lambda(x)}\right)^{\alpha}=\left(\frac{w^{+}(x, t)+\theta^{+}(x, t)}{\lambda(x)}\right)^{\alpha}=\left(A^{+}(x, t, \varphi)\right)^{\alpha}
$$

is a continuously differentiable mapping from $\mathcal{B}_{r}$ to $C_{0, s}^{\gamma, \gamma / 2}\left(\overline{\Omega_{T}^{-}}\right)$for $r \leq$ $r_{0}(\nu), T \leq T_{0}$. Besides, the following quantities do not depend on $T \in\left(0, T_{0}\right)$ for a fixed $r \leq r_{0}(\nu)$

$$
M_{1}=\sup _{\varphi \in \mathcal{B}_{r}}\left\|F^{\prime}(\varphi)\right\|_{\mathcal{B}_{r} \rightarrow Y}, \quad M_{2}=\sup _{\varphi_{1}, \varphi_{2} \in \mathcal{B}_{r}}\left\|F^{\prime}\left(\varphi_{2}\right)-F^{\prime}\left(\varphi_{1}\right)\right\|_{\mathcal{B}_{r} \rightarrow Y}, \quad T \leq T_{0} .
$$


Moreover, since the functions $\left(w^{+}, w^{-}, \sigma\right) \in C_{s}^{2+\gamma^{\prime}, 1+\gamma^{\prime} / 2}\left(\overline{\Omega_{T}^{+}}\right) \times C_{s}^{2+\gamma, 1+\gamma / 2}$ $\left(\overline{\Omega_{T}^{-}}\right) \times C^{3+\beta^{\prime}-\alpha, 1+\frac{1+\beta^{\prime}-\alpha}{2}}\left(\Gamma_{T}\right)$ with $\gamma^{\prime}>\gamma, \beta^{\prime}>\beta$, from their definition and (5.14) it follows that $\left(\psi_{0}=\left(w^{+}, w^{-}, \sigma\right)\right)$

$$
\begin{gathered}
F(0)=\left(\widetilde{F}^{+}\left(\psi_{0}\right), \widetilde{F}^{+}\left(\psi_{0}\right), \widetilde{F}^{0}\left(\psi_{0}\right)\right) \in Y^{\prime}, \\
Y^{\prime} \equiv C_{0, s}^{\gamma^{\prime}, \gamma^{\prime} / 2}\left(\overline{\Omega_{T}^{+}}\right) \times C_{0, s}^{\gamma^{\prime}, \gamma^{\prime} / 2}\left(\overline{\Omega_{T}^{-}}\right) \times C_{0}^{1+\beta^{\prime}-\alpha, \frac{1+\beta^{\prime}-\alpha}{2-\alpha}}\left(\Gamma_{T}\right) .
\end{gathered}
$$

Using inequalities (2.1)-(2.4) we have with some $\mu>0$

$$
\|F(0)\|_{Y} \leq C\left(u_{0}^{+}, u_{0}^{-}, h^{+}, h^{-}\right) T^{\mu}, \quad r \leq r_{0}(\nu), T \leq T_{0} .
$$

Thus the following lemma holds.

Lemma 5.1. Let $r_{0}(\nu), T_{0}$ are from (5.21). Let $r \leq r_{0}(\nu)$ be fixed and let $T \in\left(0, T_{0}\right)$. Then the mapping $F(\varphi)$ from (5.13) is a continuous differentiable mapping from $\mathcal{B}_{r}$ to the space $Y$ from (5.14), estimate (5.27) is valid, and $M_{1}, M_{2}$ in (5.26) do not depend on $T \in\left(0, T_{0}\right)$.

Now we give the explicit form of the Frechet derivative of $F(\varphi)$ at $\varphi=0$, that is, $F^{\prime}(0)$.

Lemma 5.2. The Frechet derivative $F^{\prime}(0)$ of the mapping $F(\varphi)$ at $\varphi=0$ is the linear mapping from $\mathcal{H}_{0}$ to $Y$ of the form $F^{\prime}(0) \varphi=\left(F^{+^{\prime}}(0) \varphi, F^{-^{\prime}}(0) \varphi, F^{0^{\prime}}(0) \varphi\right)$, where $\left(\varphi=\left(\theta^{+}, \theta^{-}, \delta\right)\right)$

$$
\begin{gathered}
F^{ \pm^{\prime}}(0) \varphi=\frac{\partial \theta^{ \pm}}{\partial t}-\left|w^{ \pm}\right|^{\alpha} \nabla_{\sigma}^{2} \theta^{ \pm}-B(x, t) \frac{\partial w^{ \pm}}{\partial \lambda}\left(\frac{\partial \delta}{\partial t}-\left|w^{ \pm}\right|^{\alpha} \nabla_{\sigma}^{2} \delta\right) \\
+\sum_{i=0}^{N}\left(a_{i}^{ \pm}(x, t) \theta_{x_{i}}^{ \pm}+b_{i}^{ \pm}(x, t) \delta_{x_{i}}\right)+a_{0}^{ \pm}(x, t) \theta^{ \pm}+b_{0}^{ \pm}(x, t) \delta+D^{ \pm}(x, t) \frac{\theta^{ \pm}(x, t)}{\lambda(x)}, \\
F^{0^{\prime}}(0) \varphi=k \delta_{t}+\left.\left[a^{+} \frac{\partial \theta^{+}}{\partial \lambda}-a^{-} \frac{\partial \theta^{-}}{\partial \lambda}\right]\right|_{\Gamma_{T}}-\left.\delta_{\lambda}\left[a^{+} \frac{\partial w^{+}}{\partial \lambda}-a^{-} \frac{\partial w^{-}}{\partial \lambda}\right]\right|_{\Gamma_{T}}+ \\
+c_{0}^{ \pm}(x, t) \theta^{ \pm}+d_{0}^{ \pm}(x, t) \delta
\end{gathered}
$$

where $B(x, t), a_{i}^{ \pm}(x, t), b_{i}^{ \pm}(x, t), a_{0}^{ \pm}(x, t), b_{0}^{ \pm}(x, t), D(x, t), c_{0}^{ \pm}(x, t), d_{0}^{ \pm}(x, t)$ are some given coefficients at the lower order terms in (5.28), (5.29), $\lambda(x)$ is from (5.17), and

$B(x, t), a_{i}^{ \pm}, b_{i}^{ \pm}, a_{0}^{ \pm}, b_{0}^{ \pm}, D^{ \pm}(x, t) \in C_{s}^{\gamma, \gamma / 2}\left(\overline{\Omega_{T}^{ \pm}}\right), \quad c_{0}^{ \pm}, d_{0}^{ \pm} \in C_{0}^{1+\beta-\alpha, \frac{1+\beta-\alpha}{2-\alpha}}\left(\Gamma_{T}\right)$,

$$
B(x, 0) \equiv 1, \quad x \in \bar{\Omega}^{ \pm} .
$$

Note that the higher order parts of differential operators acting on $\theta^{ \pm}$and $\delta$ in $(5.28),(5.29)$ are proportional to each other. Moreover, the proportionality factors are the same for $t=0$ as it follows from (5.31). This important fact will be used below.

We do not give a detailed proof of this lemma. Relation (5.29) easy follows from (4.8) and the very detailed proof of relation (5.28) is contained, in fact, 
in [6] (see [6], formula (2.26); see also [3] for another way to find the Frechet derivative). The only difference from [6] is the presence of the degenerate factor $\left|v^{ \pm}\right|^{\alpha}$ in equations (4.6). This factor is treated exactly as in (5.22)-(5.25) above. We have, for example, for $v^{+}$

$$
\begin{aligned}
& G(\varphi) \equiv\left(v^{+}\right)^{\alpha}\left[\nabla_{\rho}^{2} v^{+}\right]=\left(w^{+}+\theta^{+}\right)^{\alpha} \nabla_{\sigma+\delta}^{2}\left(w^{+}+\theta^{+}\right) \\
& \quad=\left[\left(\frac{w^{+}}{\lambda(x)}+\frac{\theta^{+}}{\lambda(x)}\right)^{\alpha}\right]\left[\lambda^{\alpha}(x) \nabla_{\sigma+\delta}^{2}\left(w^{+}+\theta^{+}\right)\right] \equiv G_{1}(\varphi) G_{2}(\varphi)
\end{aligned}
$$

The Frechet derivative of $G_{1}(\varphi)$ at $\varphi=0$ is

$$
G_{1}^{\prime}(0) \varphi=\alpha\left(\frac{w^{+}}{\lambda(x)}\right)^{\alpha-1}\left(\frac{\theta^{+}}{\lambda(x)}\right) .
$$

And the Frechet derivative of $G_{2}(\varphi)$ at $\varphi=0$ is found in [6]. So

$$
G^{\prime}(0) \varphi=\left[G_{1}^{\prime}(0) \varphi\right] G_{2}(0)+G_{1}(0)\left[G_{2}^{\prime}(0) \varphi\right]
$$

where

$$
\left[G_{1}^{\prime}(0) \varphi\right] G_{2}(0)=\left[\alpha\left(\frac{w^{+}}{\lambda(x)}\right)^{\alpha-1}\left(\frac{\theta^{+}}{\lambda(x)}\right)\right]\left[\lambda^{\alpha}(x) \nabla_{\sigma}^{2} w^{+}\right]
$$

This gives the terms $D(x, t) \frac{\theta^{ \pm}(x, t)}{\lambda(x)}$ in relations (5.29). It is important that in view of the representation (see $(5.22)-(5.25))$

$$
\frac{\theta^{+}(x, t)}{\lambda(x)}= \begin{cases}\int_{0}^{1} \frac{\partial \theta^{+}}{\partial \lambda}(\lambda s, \omega, t) d s \geq \nu, & x \in \mathcal{N}, \\ \frac{\theta^{+}}{\lambda}, & x \in \overline{\Omega^{+}} \backslash \mathcal{N},\end{cases}
$$

this term can be considered in the usual way as a lower order term. This actually completes the proof of the lemma.

\section{An initial-boundary value problem with dynamic boundary condition: the invertibility of the Frechet derivative of the operator of problem (4.6)-(4.11)}

In this section we consider the invertibility of the Frechet derivative $F^{\prime}(0)$ of the operator $F(\varphi)$ from (5.13). As in the previous section, we assume that $\lambda(x)$ is extended to all $\bar{\Omega}$ to a smooth function of the class $C^{3+\gamma}$,

$$
\nu \leq \lambda(x), d^{ \pm}(x) \leq \nu^{-1}, \quad x \in \bar{\Omega} \backslash \mathcal{N} .
$$

According to the definition of the space $\mathcal{H}_{0}$ in (5.12) and to relations (5.28), (5.29) of Lemma 5.2 we must prove solvability and estimates of the solution to the following problem for the unknown functions $\theta^{ \pm}(x, t), \delta(\omega, t)$ defined on the domains $\overline{\Omega_{T}^{ \pm}}$and on $\Gamma_{T}$ respectively (we replace $\left|w^{ \pm}\right|^{\alpha}$ in (5.28), (5.29) 
with $\lambda(x)^{\alpha} e^{ \pm}(x, t)$ and $\partial w^{ \pm} / \partial \lambda$ with $\left.A^{ \pm}(x, t)\right)$

$$
\begin{gathered}
\frac{\partial \theta^{ \pm}}{\partial t}-\lambda(x)^{\alpha} e^{ \pm}(x, t) \nabla_{\sigma}^{2} \theta^{ \pm}-B(x, t) A^{ \pm}(x, t)\left(\frac{\partial \delta}{\partial t}-\lambda(x)^{\alpha} e^{ \pm}(x, t) \nabla_{\sigma}^{2} \delta\right) \\
+\sum_{i=0}^{N}\left(a_{i}^{ \pm}(x, t) \theta_{x_{i}}^{ \pm}+b_{i}^{ \pm}(x, t) \delta_{x_{i}}\right)+a_{0}^{ \pm}(x, t) \theta^{ \pm} \\
+b_{0}^{ \pm}(x, t) \delta+D^{ \pm}(x, t) \frac{\theta^{ \pm}(x, t)}{\lambda(x)} \\
=f_{1}^{ \pm}(x, t), \quad(x, t) \in \Omega_{T}^{ \pm}, \\
\quad \theta^{+}(x, t)=\theta^{-}(x, t)=0, \quad(x, t) \in \Gamma_{T}, \\
k \delta_{t}-\varepsilon \triangle_{\Gamma} \delta+\left(a^{+} \frac{\partial \theta^{+}}{\partial \lambda}-a^{-} \frac{\partial \theta^{-}}{\partial \lambda}\right)-\delta_{\lambda}\left(a^{+} A^{+}(x, t)-a^{-} A^{-}(x, t)\right) \\
+c_{0}^{ \pm}(x, t) \theta^{ \pm}+d_{0}^{ \pm}(x, t) \delta=f_{2}(x, t), \quad(x, t) \in \Gamma_{T}, \\
\theta^{ \pm}(x, t)=0, \quad(x, t) \in \Gamma_{T}^{ \pm}, \\
\theta^{ \pm}(x, 0)=0, \quad \delta(x, 0)=0, \quad x \in \overline{\Omega^{ \pm}} \\
\delta(x, t)=E \delta(\omega, t),
\end{gathered}
$$

where the extension operator $E$ is defined in Sect. 2, $\triangle_{\Gamma}$ is the LaplaceBeltrami operator on the surface $\Gamma$. The term $\varepsilon \triangle_{\Gamma}$ is a regularisation of the problem and we need this term to prove the solvability of the problem. We assume that

$$
f_{1}^{ \pm}(x, t) \in C_{0, s}^{\gamma, \gamma / 2}\left(\overline{\Omega_{T}^{ \pm}}\right), \quad f_{2}(x, t) \in C_{0}^{1+\beta-\alpha, \frac{1+\beta-\alpha}{2-\alpha}}\left(\Gamma_{T}\right),
$$

$\varepsilon, a^{ \pm}, k$ are given positive constants,

$$
\begin{gathered}
\nu \leq k, a^{ \pm}, e^{ \pm}(x, t), B(x, t), \quad A^{ \pm}(x, t) \leq \nu^{-1}, B(x, 0) \equiv 1, \\
B(x, t), D^{ \pm}(x, t) \in C_{s}^{\gamma, \gamma / 2}\left(\bar{\Omega}_{T}^{ \pm}\right), A^{ \pm}(x, t) \in C^{1+\beta-\alpha, \frac{1+\beta-\alpha}{2-\alpha}}\left(\bar{\Omega}_{T}^{ \pm}\right),
\end{gathered}
$$

and the functions $a_{i}^{ \pm}, b_{i}^{ \pm}, a_{0}^{ \pm}, b_{0}^{ \pm}, D^{ \pm}(x, t), c_{0}^{ \pm}, d_{0}^{ \pm}$satisfy (5.30).

We can obtain the Schauder a priori estimates of the solution for problem (6.2)-(6.7) by the standard method. This method consists in the dropping the lower order terms in (6.2), (6.4), the freezing coefficients, and multiplying by smooth cutting functions. This can be done completely similar to [43] (or [6] in the case of Stefan problem). The principal model problem obtained by the freezing of the coefficients at points of the boundary $\Gamma$ at $t=0$ (with the subsequent local rectification of the boundary) is studied in Sect. 3. At considering such a model problem, the functions $e^{ \pm}(x, t)$ and $A^{ \pm}(x t)$ are replaced by the constants $e^{ \pm} \equiv e^{ \pm}\left(x_{0}, 0\right)$ and $A^{ \pm} \equiv A^{ \pm}\left(x_{0}, 0\right), x_{0} \in \Gamma$. After this, in view of the condition $B(x, 0) \equiv 1$, the change of the unknown function

$$
u^{ \pm}(x, t)=\theta^{ \pm}(x, t)-A^{ \pm} \delta
$$


reduces problem (6.2)-(6.7) with the frozen coefficients and with the flat boundary exactly to problem (3.3)-(3.7). From these model problems associated with the boundary $\Gamma$ we get an estimate of the function $\left.\delta(x, t)\right|_{\Gamma}$ and estimates of the functions $\theta^{ \pm}(x, t)$ near the boundary $\Gamma_{T}$. The rest of the model problems associated with strictly interior points of $\Omega^{ \pm}$are standard because of the condition (6.7) and due to the absence of degeneracy of the equations at these points - see [43]. Thus we have the following assertion.

Lemma 6.1. Suppose that conditions (6.8)-(6.10) are satisfied. Then there exists sufficiently small $T_{0}=T_{0}(B)$ such that for $T \leq T_{0}$ for the solution of problem (6.2)-(6.7) from the class $\theta^{ \pm} \in C_{s}^{2+\gamma, \frac{2+\gamma}{2}}\left(\bar{\Omega}_{T}^{ \pm}\right), \delta \in$ $C^{3+\beta-\alpha, 1+\frac{1+\beta-\alpha}{2-\alpha}}\left(\Gamma_{T}\right)$ the following a priori estimate is valid

$$
\begin{aligned}
\left|\theta^{ \pm}\right|_{s, \bar{\Omega}_{T}^{ \pm}}^{(2+\gamma)} & +|\delta|_{\Gamma_{T}}^{\left(2+\beta-\alpha, \frac{2+\beta-\alpha}{2-\alpha}\right)}+\left|\delta_{t}\right|_{\Gamma_{T}}^{\left(1+\beta-\alpha, \frac{1+\beta-\alpha}{2-\alpha}\right)}+\varepsilon \sum_{i, j=1}^{N-1}\left|\delta_{\omega_{i} \omega_{j}}\right|_{\Gamma_{T}}^{\left(1+\beta-\alpha, \frac{1+\beta-\alpha}{2-\alpha}\right)} \\
& \leq C_{T}\left(\left|f_{1}^{+}\right|_{s, \bar{\Omega}_{T}^{+}}^{(\gamma)}+\left|f_{1}^{-}\right|_{s, \bar{\Omega}_{T}^{-}}^{(\gamma)}+\left|f_{2}\right|_{\Gamma_{T}}^{\left(1+\beta-\alpha, \frac{1+\beta-\alpha}{2-\alpha}\right)}\right) \equiv C_{T} \mathcal{M}(T),
\end{aligned}
$$

where the constant $C_{T}$ in (6.12) does not depend on $\varepsilon \in(0,1)$.

If $B(x, t) \equiv 1$ then $T_{0}=\infty$.

Let us remark that the condition $B(x, t) \equiv 1$ permits us to make change of the unknown functions (6.11) for any $t=t_{0}>0$ not only for $t_{0}=0$. So we have the estimate of the lemma for any $T>0$.

We now show the solvability of problem (6.2)-(6.7).

Theorem 6.2. Suppose that conditions (6.8)-(6.10) are satisfied. Then for $T \leq$ $T_{0}, \varepsilon \in(0,1)$ problem $(6.2)-(6.7)$ is solvable in the space $\theta^{ \pm} \in C_{s}^{2+\gamma, \frac{2+\gamma}{2}}\left(\bar{\Omega}_{T}^{ \pm}\right)$, $\delta \in C^{3+\beta-\alpha, 1+\frac{1+\beta-\alpha}{2-\alpha}}\left(\Gamma_{T}\right)$ and estimate (6.12) of the solution is valid.

When $\varepsilon=0$ problem $(6.2)-(6.7)$ is solvable in the space $\theta^{ \pm} \in$ $C_{s}^{2+\gamma, \frac{2+\gamma}{2}}\left(\bar{\Omega}_{T}^{ \pm}\right), \delta \in C^{2+\beta-\alpha, \frac{2+\beta-\alpha}{2-\alpha}}\left(\Gamma_{T}\right), \delta_{t} \in C^{1+\beta-\alpha, \frac{1+\beta-\alpha}{2-\alpha}}\left(\Gamma_{T}\right)$ and estimate (6.12) without the term with $\varepsilon$ is valid.

If $B(x, t) \equiv 1$ then the above assertions are valid for any $T>0$.

Proof. Define a linear operator $M: \delta \rightarrow \theta^{ \pm} \rightarrow M \delta$ in the following way. Let $M$ first maps a function $\delta \in C^{2+\beta-\alpha, \frac{2+\beta-\alpha}{2-\alpha}}\left(\Gamma_{T}\right)$ to the functions $\theta^{ \pm}$as the solution of the problem (6.2)-(6.6) with the given function $\delta$ in (6.2). And then the operator $M$ maps the functions $\theta^{ \pm}$to the function $M \delta$ and the last is determined from the condition (6.4) with the given $\theta^{ \pm}, \delta_{\lambda}$ and $\delta$ in lower order term. That is, the function $M \delta$ is the solution of the problem

$$
\begin{gathered}
k(M \delta)_{t}-\varepsilon \Delta_{\Gamma}(M \delta)=f_{2}-\left(a^{+} \frac{\partial \theta^{+}}{\partial \lambda}-a^{-} \frac{\partial \theta^{-}}{\partial \lambda}\right)+\delta_{\lambda}\left(a^{+} A^{+}-a^{-} A^{-}\right)- \\
-\left(c_{0}^{ \pm}(x, t) \theta^{ \pm}+d_{0}^{ \pm}(x, t) \delta\right), \\
M \delta(\omega, 0)=0 .
\end{gathered}
$$


By Theorem 2.7, this operator is well defined. By the known properties of the problem (6.13) we have for $\varepsilon>0$

$$
\begin{gathered}
|M \delta|_{\Gamma_{T}}^{\left(3+\beta-\alpha, 1+\frac{1+\beta-\alpha}{2-\alpha}\right)} \leq C_{\varepsilon, T}\left(|\delta|_{\Gamma_{T}}^{\left(2+\beta-\alpha, \frac{2+\beta-\alpha}{2-\alpha}\right)}+\mathcal{M}(T)\right), \\
\left|M \delta_{2}-M \delta_{1}\right|_{\Gamma_{T}}^{\left(3+\beta-\alpha, 1+\frac{1+\beta-\alpha}{2-\alpha}\right)} \leq C_{\varepsilon, T}\left|\delta_{2}-\delta_{1}\right|_{\Gamma_{T}}^{\left(2+\beta-\alpha, \frac{2+\beta-\alpha}{2-\alpha}\right)} .
\end{gathered}
$$

Consequently, by (2.4)

$$
\begin{gathered}
\left|M \delta_{2}-M \delta_{1}\right|_{\Gamma_{T}}^{\left(2+\beta-\alpha, \frac{2+\beta-\alpha}{2-\alpha}\right)} \leq C T^{\mu}\left|M \delta_{2}-M \delta_{1}\right|_{\Gamma_{T}}^{\left(3+\beta-\alpha, 1+\frac{1+\beta-\alpha}{2-\alpha}\right)} \leq \\
\leq C_{\varepsilon, T} T^{\mu}\left|\delta_{2}-\delta_{1}\right|_{\Gamma_{T}}^{\left(2+\beta-\alpha, \frac{2+\beta-\alpha}{2-\alpha}\right)} .
\end{gathered}
$$

Thus for a sufficiently small $T=T_{\varepsilon}$ the operator $M$ is a contraction on $C_{0}^{2+\beta-\alpha, \frac{2+\beta-\alpha}{2-\alpha}}\left(\Gamma_{T}\right)$ and therefore has a unique fixed point. By $(6.14)$ this fixed point belongs also to the space $C_{0}^{3+\beta-\alpha, 1+\frac{1+\beta-\alpha}{2-\alpha}}\left(\Gamma_{T}\right)$ and together with the corresponding $\theta^{ \pm}$gives the solution of the problem. The estimate of the solution is given by Lemma 6.1. Moving now step by step up the axis $O t$ as in [43], we obtain the theorem with $\varepsilon>0$ for any $T \leq T_{0}$.

Consider now the sequence of the solutions $v_{\varepsilon}^{ \pm}, \delta_{\varepsilon}, \varepsilon \rightarrow 0$. From estimate (6.12) it follows that this sequence is a compact set in the spaces $C_{0, s}^{2+\bar{\gamma}, \frac{2+\bar{\gamma}}{2}}\left(\bar{\Omega}_{T}^{ \pm}\right)$ and $C_{0}^{2+\bar{\beta}-\alpha, \frac{2+\bar{\beta}-\alpha}{2-\alpha}}\left(\Gamma_{T}\right)$ correspondingly for any $\bar{\gamma}<\gamma, \bar{\beta}=\bar{\gamma}(1-\alpha / 2)$. The passing to the limit of this sequence in the spaces $C_{0, s}^{2+\bar{\gamma}, \frac{2+\bar{\gamma}}{2}}\left(\bar{\Omega}_{T}^{ \pm}\right)$and $C_{0}^{2+\bar{\beta}-\alpha, \frac{2+\bar{\beta}-\alpha}{2-\alpha}}\left(\Gamma_{T}\right), \delta_{t} \in C_{0}^{2+\bar{\beta}-\alpha, \frac{2+\bar{\beta}-\alpha}{2-\alpha}}\left(\Gamma_{T}\right)$ gives the solution of problem (6.2)-(6.7) for $\varepsilon=0$. Besides, from uniform in $\varepsilon$ estimate (6.12) it follows that the limit functions $v^{ \pm}$and $\delta$ belong to the spaces $C_{0, s}^{2+\gamma, \frac{2+\gamma}{2}}\left(\bar{\Omega}_{T}^{ \pm}\right)$and $C_{0}^{2+\beta-\alpha, \frac{2+\beta-\alpha}{2-\alpha}}\left(\Gamma_{T}\right), \delta_{t} \in C_{0}^{2+\beta-\alpha, \frac{2+\beta-\alpha}{2-\alpha}}\left(\Gamma_{T}\right)$ correspondingly.

Thus Theorem 6.2 is proved.

From this theorem, Lemma 5.2, and the definition of the space $\mathcal{H}_{0}$ we obtain the following assertion.

Corollary 6.3. Let the mapping $F(\varphi)$ is defined in (5.13) and $T_{0}$ is defined in Lemma 5.1. The Frechet derivative $F^{\prime}(0)$ is invertible operator and

$$
\left\|\left(F^{\prime}(0)\right)^{-1}\right\|_{Y \rightarrow \mathcal{H}_{0}} \leq C_{T_{0}},
$$

where the constant $C_{T_{0}}$ does not depend on $T \leq T_{0}$.

As a corollary of Theorem 6.2, Lemma 6.1 and their proofs we can obtain the following assertion. Let $V$ be a domain in $R^{N}$ with boundary $S$ of the class $C^{2+\gamma}, V_{T}=V \times[0, T], S_{T}=S \times[0, T]$. Consider the following initial-boundary value problem with a dynamic boundary condition for a unknown function $u(x, t)$

$$
\frac{\partial u(x, t)}{\partial t}-d^{\alpha}(x) \nabla^{2} u(x, t)=f_{1}(x, t), \quad(x, t) \in V_{T},
$$




$$
\begin{gathered}
\frac{\partial u(x, t)}{\partial t}-\varepsilon \Delta_{S} u(x, t)+\frac{\partial u(x, t)}{\partial \vec{n}}=f_{2}(x, t), \quad(x, t) \in S_{T}, \\
u(x, 0)=u_{0}(x) .
\end{gathered}
$$

Here $\varepsilon \in[0,1), \Delta_{S}$ is the Laplace-Beltrami operator on $S, d(x)$ satisfies conditions

$$
\nu \operatorname{dist}(x, S) \leq d(x) \leq \nu^{-1} \operatorname{dist}(x, S), \quad \nu>0,
$$

and

$$
\begin{gathered}
d(x) \in C^{1+\gamma}(\bar{V}) \\
f_{1} \in C_{s}^{\gamma, \gamma / 2}\left(\bar{V}_{T}\right), f_{2} \in C^{1+\beta-\alpha, \frac{1+\beta-\alpha}{2-\alpha}}\left(S_{T}\right), u_{0} \in C^{2+\gamma}(\bar{V}),\left.u_{0}\right|_{S} \in C^{3+\gamma}(\bar{V}) .
\end{gathered}
$$

Theorem 6.4. Let conditions (6.20), (6.21) are satisfied. Then for $\varepsilon>0$ problem (6.17)-(6.19) has a unique solution $u(x, t)$ from the class $u \in$ $C_{s}^{2+\gamma, \frac{2+\gamma}{2}}\left(\bar{V}_{T}\right),\left.u\right|_{S_{T}} \in C^{3+\beta-\alpha, 1+\frac{1+\beta-\alpha}{2-\alpha}}\left(\Gamma_{T}\right)$ and the estimate

$$
\begin{aligned}
& |u|_{s, V_{T}}^{(2+\gamma)}+\left.\left|u_{t}\right|_{S_{T}}\right|_{S_{T}} ^{\left(1+\beta-\alpha, \frac{1+\beta-\alpha}{2-\alpha}\right)}+\varepsilon \sum_{i, j=1}^{N-1}\left|\left(\left.u\right|_{S_{T}}\right)_{\omega_{i} \omega_{j}}\right|_{S_{T}}^{\left(1+\beta-\alpha, \frac{1+\beta-\alpha}{2-\alpha}\right)} \leq \\
& \leq C_{T}\left(\left|f_{1}\right|_{s, \bar{V}_{T}}^{(\gamma)}+\left|f_{2}\right|_{S_{T}}^{\left(1+\beta-\alpha, \frac{1+\beta-\alpha}{2-\alpha}\right)}\right)
\end{aligned}
$$

is valid. When $\varepsilon=0$ problem $(6.17)-(6.19)$ is solvable in the space $u \in$ $C_{s}^{2+\gamma, \frac{2+\gamma}{2}}\left(\bar{\Omega}_{T}^{ \pm}\right),\left.u_{t}\right|_{S_{T}} \in C^{1+\beta-\alpha, \frac{1+\beta-\alpha}{2-\alpha}}\left(\Gamma_{T}\right)$ and estimate $(6.22)$ without the term with $\varepsilon$ is valid.

We do not give the proof of this theorem as it exactly coincides with the proofs of Lemma 6.1 and Theorem 6.2 with the help of Theorem 3.4. The difference is the presence of the nonzero initial data in (6.19). This case is reduced to the case of the zero initial data and to the spaces with zeros in (6.21) for $f_{1}, f_{2}$ by results of paper [42]. Besides, to obtain estimate (6.22) one should use Theorem 3.4 as a simplified version of Theorem 3.1.

\section{Completion of the proof of Theorem 1.1}

We complete the proof of Theorem 1.1 by Corollary 1.3. Consider the mapping $F(\varphi)$ on $\mathcal{B}_{r}$, where $F(\varphi)$ is defined in (5.13) and $\mathcal{B}_{r}$ is defined in (5.15). From Lemma 5.1 and Corollary 6.3 it follows that the conditions of Corollary 1.3 are satisfied except may be for the condition $\|F(0)\|_{Y} \leq \varepsilon_{0}$. But again from Lemma 5.1 we have

$$
\|F(0)\|_{Y} \leq C T^{\mu}, \quad T \leq T_{0},
$$

where $T_{0}$ is defined in Corollary 6.3. So by decreasing the value of $T_{0}$ we can obtain all conditions of Corollary 1.3. Consequently, there exists $\varphi^{*}=$ $\left(\theta^{+*}, \theta^{-*}, \delta^{*}\right) \in \mathcal{B}_{r}$ such that $F\left(\varphi^{*}\right)=0$. Then the triple $\left(v^{+}, v^{-}, \rho\right)=\left(w^{+}+\right.$ 
$\left.\theta^{+*}, w^{-}+\theta^{-*}, \sigma+\delta^{*}\right)$ gives a solution of problem (4.6)-(4.11) and so a solution of the original nonlinear problem with free boundary.

Let us explain now the uniqueness of the solution of the problem for a sufficiently small time interval $[0, T]$. From Theorem 1.2 it follows that the ball $\mathcal{B}_{r}$ contains only one solution of the equation $F(\varphi)=0$ if it's radius $r$ is sufficiently small. Let $\overline{\mathcal{H}}_{0}$ and $\bar{Y}$ are the spaces $\mathcal{H}_{0}, Y$ with exponents $\bar{\gamma}<\gamma$, $\bar{\beta}<\beta$ instead of $\gamma, \beta$. Let $\left(v_{i}^{+}, v_{i}^{-}, \rho_{i}\right), i=1,2$ are two solutions of (4.6)(4.11) from the desired classes. Let also $\varphi_{i}, i=1,2$ are the two corresponding elements of $\mathcal{H}_{0}$. From (2.1)-(2.4) it follows that

$$
\left\|\varphi_{i}\right\|_{\overline{\mathcal{H}}_{0}} \leq C T^{\mu}\left\|\varphi_{i}\right\|_{\mathcal{H}_{0}} .
$$

This means that for arbitrary small $T>0$ both solutions $\varphi_{1}, \varphi_{2}$ belong to the ball $\overline{\mathcal{B}}_{r}(0) \subset \overline{\mathcal{H}}_{0}$ of arbitrary small radius $r$. Consider now the mapping $F(\varphi)$ as a mapping from the space $\overline{\mathcal{H}}_{0}$ to $\bar{Y}$. Evidently, all the proved above properties of $F(\varphi)$ are preserved for such mapping. Particularly, we must have $\varphi_{1}=\varphi_{2}$ if the radius $r$ of $\overline{\mathcal{B}}_{r}(0)$ is sufficiently small, which is the case if the time interval $[0, T]$ is sufficiently small.

Thus Theorem 1.1 is proved.

\section{References}

[1] Meirmanov, A.M.: On the classical solution of the multidimensional Stefan problem for quasilinear parabolic equations. Math. USSR Sb. 40(2), 157-178 (1981)

[2] Hanzawa, E.-I.: Classical solutions of the Stefan problem. Tohoku Math. J. 33, 297-335 (1981)

[3] Bazalii, B.V., Degtyarev, S.P.: On classical solvability of the multidimensional Stefan problem for convective motion of a viscous incompressible fluid. Math. USSR Sb. 60(1), 1-17 (1988)

[4] Radkevich, E.V.: On conditions for the existence of a classical solution of the Stefan contact problem Math. USSR Sb. 69(2), 497-525 (1991)

[5] Borodin, M.A.: Existence of the global classical solution for a two-phase Stefan problem. SIAM J. Math. Anal. 30(6), 1264-1281 (1999)

[6] Bizhanova, G.I., Solonnikov, V.A.: On problems with free boundaries for secondorder parabolic equations. St. Petersburg Math. J. 12(6), 949-981 (2001)

[7] Kusaka, Y., Tani, A.: On the classical solvability of the stefan problem in a viscous incompressible fluid flow. SIAM J. Math. Anal. 30(3), 584-602 (1999)

[8] Yi, F., Liu, Y.: Two-phase stefan problem as the limit case of two-phase Stefan problem with kinetic condition. J. Differ. Equ. 183(1), 189-207 (2002)

[9] Daskalopoulos, P., Lee, K.-A.: All time smooth solutions of the one-phase Stefan problem and the Hele-Shaw flow. Commun. Partial Differ. Equ. 29(1-2), 71$89(2005)$ 
[10] Friedman, A., Hu, B., Velazquez, J.J.L.: A Stefan problem for a protocell model with symmetry-breaking bifurcations of analitic solutions. Interfaces Free Bound 3(2), 143-199 (2001)

[11] Friedman, A., Velazquez, J.J.L.: A free boundary problem associated with crystallization of polymers in a temperature field. Indiana Univ. Math. J. 50(4), 1609-1649 (2001)

[12] Prüss, J., Simonett, G., Zacher, R.: Qualitative behavior of solutions for thermodinamically consistent Strfan problems. Arch. Ration. Mech. Anal. 207(2), 511-667 (2013)

[13] Kusaka, Y.: Global-in-time strong solvability of the multi-dimensional onephase Stefan problem for an incompressible viscous fluid. Jpn. J. Ind. Appl. Math. 30(2), 415-439 (2013)

[14] Visintin, A.: The Stefan problem for a class of degenerate parabolic equations. Free boundary problems: theory and applications, II. Research Notes in Mathematics, vol. 79, pp. 419-430. Pitman Advanced Publishing Program, Boston (1983)

[15] Bertsch, M., De Mottoni, P., Peletier, L.A.: Degenerate diffusion and the Stefan problem. Nonlinear Anal. TMA 8, 1311-1336 (1984)

[16] Cannon, J.R., Hong-Ming, Y.: On the existence of the weak solution and the regularity of the free boundary to a one-dimensional two-phase degenerate Stefan problem. J. Differ.Equ. 73(1), 104-118 (1988)

[17] Xiangshen, X., Shillor, M.: The Stefan problem with convection and Joule's heating. Adv. Differ. Equ. 2(4), 667-691 (1997)

[18] Urbano, J.M.: On the Stefan problem with convection and nonlinear diffusion in a porous medium. Nonlinear Evolution Equations and Their Applications, pp. 225-236. World Scientific, Singapore (1999)

[19] Guangwei, Y.: Local existence of bounded solutions to the degenerate Stefan problem with Joule's heating. J. Partial Differ. Equ. 9(1), 42-54 (1996)

[20] Di Benedetto, E.: Degenerate Parabolic Equations. Springer-Verlag, New York (1993)

[21] Bazaliy, B.V., Danilyuk, I.I., Degtyarev, S.P.: Classical solvability of some free boundary problems for parabolic equations with degeneration. Free boundary problems involving solid. Longman Sci. Tech. Pitman. Res. Notes Math. Ser. 281, 88-91 (1993)

[22] Bazalii, B.V., Degtyarev, S.P.: Degenerate parabolic equations and problems with a free boundary. (Russian) Dokl. Akad. Nauk Ukrain. SSR Ser. A 1, 3-7 (1990)

[23] Xiangsheng, X.: Existence and regularity theorems for a two-phase degenerate Stefan problem with convection. Appl. Anal. 51(1-4), 221-250 (1993) 
[24] Huilai, L.: A degenerate Stefan problem with two free boundaries. Northeast. Math. J. 11(3), 263-274 (1995)

[25] Dening, L.: Regularity of solutions for a two-phase degenerate Stefan problem. J. Differ.Equ. 102(2), 402-418 (1993)

[26] Dancer, E.N., Hilhorst, D., Mimura, M., Peletier, L.A.: Spatial segregation limit of a competition-diffusion system. Eur. J. Appl. Math. 10(2), 97-115 (1999)

[27] Weller, F.F.: A free boundary problem modeling thrombus growth. Model development and numerical simulation using the level set method. J. Math. Biol. 61(6), 805-818 (2010)

[28] Weller, F.F., Neuss-Radu, M., Jger, W.: Analysis of a free boundary problem modeling thrombus growth. SIAM J. Math. Anal. 45(2), 809-833 (2013)

[29] Zhao, J., Wang, M.: A free boundary problem of a predator-prey model with higher dimension and heterogeneous environment. Nonlinear Anal. Real World Appl. 16, 250-263 (2014)

[30] Athreya, S.R., Barlow, M.T., Bass, R.F., Perkins, E.A.: Degenerate stochastic differential equations and super-Markov chains. Probab. Theory Related Fields 123(4), 484-520 (2002)

[31] Epstein, C.L., Mazzeo, R.: Degenerate Diffusion Operators Arising in Population Biology. Princeton Uni-versity Press, Princeton, arXiv:1110.0032 (2013)

[32] van Moerbeke, P.: An optimal stopping problem with linear reward. Acta Math. 132, 111-151 (1974)

[33] Bensoussan, A., Friedman, A.: Nonzero-sum stochastic differential games with stopping times and free boundary problems. Trans. Am. Math. Soc. 231(2), 275$327(1977)$

[34] Denk, R., Prüss, J., Zacher, R.: Maximal $L_{p}$ : regularity of parabolic problems with boundary dynamics of relaxation type. J. Funct. Anal. 255(11), 31493187 (2008)

[35] Escher, J.: Quasilinear parabolic systems with dynamical boundary conditions. Comm. Partial Differ. Equ. 18(7-8), 1309-1364 (1993)

[36] Prüss, J., Racke, R., Zheng, S.: Maximal regularity and a symptotic behavior of solutions for the Cahn-Hilliard equation with dynamic boundary conditions. Ana. Mate. 185(4), 627-648 (2006)

[37] Goldstein, G.R., Miranville, A.: A Cahn-Hilliard-Gurtin model with dynamic boundary conditions. Discrete Contin. Dyn. Syst. Ser. S 6(2), 387-400 (2013)

[38] Vázquez, J.L., Vitillaro, E.: On the Laplace equation with dynamical boundary conditions of reactive-diffusive type. J. Math. Anal. Appl. 354(2), 674-688 (2009)

[39] Vázquez, J.L., Vitillaro, E.: Heat equation with dynamical boundary conditions of reactive type. Commun. Partial Differ. Equ. 33(4-6), 561-612 (2008) 
[40] Frolova, E.: Solvability in Sobolev spaces of a problem for a second order parabolic equation with time derivative in the boundary condition. Portugal Math. 56(4), 419-441 (1999)

[41] Rodrigues, J.F., Solonnikov, V.A.: On a parabolic system with time derivative in the boundary conditions and related free boundary problems. Math. Ann. 315(1), 61-95 (1999)

[42] Kim, S., Lee, K.-A.: Smooth solution for the porous medium equation in a bounded domain. J. Differ. Equ. 247(4), 1064-1095 (2009)

[43] Ladyzhenskaja, O.A., Solonnikov, V.A., Uraltseva, N.N.: Linear and quasilinear equations of parabolic type. Translations of Mathematical Monographs, vol. 23, xi+648. American Mathematical Society, Providence (1968)

[44] Daskalopoulos, P., Hamilton, R.: Regularity of the free boundary for the porous medium equation. J. Am. Math. Soc. 11(4), 899-965 (1998)

[45] Solonnikov, V.A.: Estimates for solutions of a non-stationary linearized system of Navier-Stokes equations (in Russian). Trudy Mat. Inst. Steklov (Proc. Steklov Inst. Math.) 70, 213-317 (1964)

[46] Solonnikov, V.A.: Solvability of a problem on the motion of a viscous incompressible fluid bounded by a free surface. Math. USSR Izv. 11(6), 1323-1358 (1977)

[47] Bazaliy, B.V., Friedman, A.: A free boundary problem for an elliptic-parabolic system: application to a model of tumor growth. Commun. Partial Differ. Equ. 28(3-4), 517-560 (2003)

[48] Bazaliy, B.V., Friedman, A.: The Hele-Shaw problem with surface tension in a half-plane. J. Differ. Equ. 216(2), 439-469 (2005)

[49] Bazaliy, B.V., Degtyarev, S.P.: Classical solutions of many-dimensional ellipticparabolic free boundary problems. Nonlinear Differ. Equ. Appl. 16(4), 421-443 (2009)

[50] Lunardi, A.: Analitic semigroups and optimal regularity in parabolic problems. Progress in Nonlinear Differential Equations and their Applications, vol. 16. Birkhäuser (1995)

[51] Lange, S.: Real and Functional Analysis. Graduate Texts in Mathematics, vol. 142, pp. xiv+580. Springer-Verlag, New York (1993)

[52] Bizhanova, G.I.: Investigation of solvability of the multidimensional two-phase Stefan and the nonstationary filtration Florin problems for second order parabolic equations in weighted Hölder spaces of functions. J. Math. Sci. 84(1), 823844 (1997)

[53] Brandt, A.: Interior Schauder estimates for parabolic differential: (or difference-) equations via the maximum principle. Isr. J. Math. 7, 254-262 (1969)

[54] Golovkin, K.K.: On equivalent normalizations of fractional spaces (Russian). Trudy Mat. Inst. Steklov (Proc. Steklov Inst. Math.) 66, 364-383 (1962) 
[55] Bazalii, B.V., Degtyarev, S.P.: A boundary-value problem in weighted Hölder spaces for elliptic equations which degenerate at the boundary of the domain. Sbornik Math. 204(7), 958-978 (2013)

[56] Ladyzhenskaya, O.A., Uraltseva, N.N.: Linear and Quasilinear Equations of Elliptic Type. Second edn. "Nauka", Moscow, p. 576 (1973)

[57] Solonnikov, V.A.: General boundary value problems for systems elliptic in the sense of A. Douglis and L. Nirenberg. II. (Russian). Trudy Mat. Inst. Steklov (Proc. Steklov Inst. Math.) 92, 233-297 (1966)

[58] Stein, E.M.: Singular Integrals and Differentiability Properties of Functions, Princeton Mathematical Series, No. 30, pp. xiv+290. Princeton University Press, Princeton (1970)

[59] Friedman, A.: Partial Differential Equations of Parabolic Type, pp. xiv+347. Prentice-Hall Inc., Englewood Cliffs (1964)

S. P. Degtyarev

Institute of Applied Mathematics and Mechanics NASU

R.Luxemburg str. 74

83114 Donetsk

Ukraine

e-mail: degtyar@i.ua

Received: 25 January 2014.

Accepted: 16 June 2014. 